\title{
Measurement of $\mathrm{VH}, \mathbf{H} \rightarrow \mathbf{b} \overline{\mathbf{b}}$ production as a function of the vector-boson transverse momentum in $13 \mathrm{TeV}$ pp collisions with the ATLAS detector
}

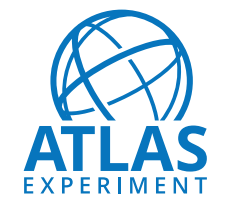

\section{The ATLAS collaboration}

\section{E-mail: atlas.publications@cern.ch}

ABSTRACT: Cross-sections of associated production of a Higgs boson decaying into bottomquark pairs and an electroweak gauge boson, $W$ or $Z$, decaying into leptons are measured as a function of the gauge boson transverse momentum. The measurements are performed in kinematic fiducial volumes defined in the 'simplified template cross-section' framework. The results are obtained using $79.8 \mathrm{fb}^{-1}$ of proton-proton collisions recorded by the ATLAS detector at the Large Hadron Collider at a centre-of-mass energy of $13 \mathrm{TeV}$. All measurements are found to be in agreement with the Standard Model predictions, and limits are set on the parameters of an effective Lagrangian sensitive to modifications of the Higgs boson couplings to the electroweak gauge bosons.

KEYWORDS: Hadron-Hadron scattering (experiments), Higgs physics

ARXIV EPRINT: 1903.04618 


\section{Contents}

1 Introduction 1

2 Data and simulation samples 2

3 Event selection and categorisation 3

4 Cross-section measurements 4

5 Results 9

6 Constraints on anomalous Higgs boson interactions 11

$\begin{array}{lll}7 & \text { Conclusion } & 13\end{array}$

$\begin{array}{ll}\text { The ATLAS collaboration } & 19\end{array}$

\section{Introduction}

A particle consistent with the Standard Model (SM) predictions for the Higgs boson [1-4] was observed in 2012 by the ATLAS and CMS collaborations [5, 6] at the LHC. Further analysis of ATLAS and CMS data collected in proton-proton $(p p)$ collisions at centre-ofmass energies of $7 \mathrm{TeV}, 8 \mathrm{TeV}$ and $13 \mathrm{TeV}$ in two LHC data-taking periods (Runs 1 and 2) has led to precise measurements of the mass of this particle (around $125 \mathrm{GeV}$ ) [7-9], tests of its spin and parity $\left(J^{P}=0^{+}\right)$against alternative hypotheses [10,11], as well as to measurements of its production and decay rates [12-14].

Recently, experiments at the LHC observed Higgs boson production in association with weak gauge bosons $V=W, Z$ ( $V H$ production) [15] and Higgs boson decays into pairs of bottom quarks $(H \rightarrow b \bar{b})[15,16]$. With these results, the four most important Higgs boson production modes predicted by the SM, gluon-gluon fusion $(\mathrm{ggF})$, vector-boson fusion $(\mathrm{VBF})$, and associated production of a Higgs boson with either a weak gauge boson $(V H)$ or a top-quark pair $(t \bar{t} H)$ are established. Similarly, several of the main modes of Higgs boson decays into fermionic $(b \bar{b}, \tau \tau)$ and bosonic $(W W, Z Z, \gamma \gamma)$ final states are observed. All results, typically expressed in the form of 'signal strengths', defined as the ratio of the observed to the expected product of the production cross-section times branching ratio into a certain final state, are consistent with SM predictions within uncertainties.

To probe the kinematic properties of Higgs boson production in more detail, to reduce the impact of theoretical uncertainties on the measurements and to make the measurements easier to compare with future updated calculations, the framework of simplified template cross-sections (STXS) has been introduced $[17,18]$. In this framework, the cross-sections 
for the various Higgs boson production modes are measured in exclusive regions carefully defined by fiducial selections based on the kinematic properties of Higgs boson production. The extrapolation from the phase space selected by the analysis criteria to that for which the cross-section measurements are presented is thus reduced.

The STXS measurements are designed to proceed in stages of increasing granularity with more recorded data. In 'stage 0', cross-sections are measured separately for the four main production modes in a fiducial Higgs boson rapidity region $\left|y_{H}\right|<2.5{ }^{1}$ mainly driven by the ATLAS and CMS detector acceptances for most of the reconstructed objects (leptons, photons and $b$-jets). In 'stage 1 ' these regions are split into 31 subregions according to kinematic properties such as the number of particle-level jets with transverse momentum $p_{\mathrm{T}}>30 \mathrm{GeV}$ (excluding any jets from Higgs boson decays), the transverse momentum of the Higgs boson, or the transverse momentum of the weak gauge boson $V$ for $V H, V \rightarrow$ leptons production. In simulation, particle-level jets are built by clustering all generated stable particles $(c \tau>10 \mathrm{~mm})$, excluding the decay products of the Higgs boson as well as the neutrinos and charged leptons from the decays of the weak gauge boson, using the anti- $k_{t}$ clustering algorithm [19] with a radius parameter $R=0.4$.

Stage-0 STXS were measured recently with $36.1 \mathrm{fb}^{-1}$ of $13 \mathrm{TeV}$ ATLAS data using $H \rightarrow$ $\gamma \gamma[20]$ and $H \rightarrow Z Z^{*} \rightarrow 4 \ell$ decays [21], with results in agreement with SM predictions. In addition, refs. [20] and [21] contain some 'reduced' stage-1 STXS measurements of ggF and VBF regions, after merging together regions where the data lack sufficient sensitivity to Higgs boson production. Given the low $V H$ production cross-sections, the only Higgs boson decay mode that can currently be measured is $H \rightarrow b \bar{b}$, with its large branching ratio of $58 \%$. This paper presents a measurement of 'reduced' stage-1 $V H$ STXS (defined in section 3) using $H \rightarrow b \bar{b}$ decays with $79.8 \mathrm{fb}^{-1}$ of $13 \mathrm{TeV} p p$ collisions collected by ATLAS between 2015 and 2017. The results are used to investigate the strength and tensor structure of the interactions of the Higgs boson with vector bosons using an effective Lagrangian approach [22].

\section{Data and simulation samples}

The data were collected with the ATLAS detector [23, 24] between 2015 and 2017, triggered by isolated charged leptons or large transverse momentum imbalance, $E_{\mathrm{T}}^{\mathrm{miss}}$. Only events with good data quality were kept.

The Monte Carlo simulation samples used for the measurements presented here are identical to those used for the measurement of the inclusive $V H, H \rightarrow b \bar{b}$ signal strength [15]. Several samples of simulated events were produced for the signal ( $q \bar{q} \rightarrow W H$, $q \bar{q} \rightarrow Z H$ and $g g \rightarrow Z H$ ) and main background ( $t \bar{t}$, single-top, $V+$ jets and diboson) processes. They were used to optimise the analysis criteria and to determine the expected

\footnotetext{
${ }^{1}$ ATLAS uses a right-handed coordinate system with its origin at the nominal interaction point (IP) in the centre of the detector and the $z$-axis along the beam pipe. The $x$-axis points from the IP to the centre of the LHC ring, and the $y$-axis points upwards. Cylindrical coordinates $(r, \phi)$ are used in the transverse plane, $\phi$ being the azimuthal angle around the $z$-axis. The pseudorapidity is defined in terms of the polar angle $\theta$ as $\eta=-\ln \tan (\theta / 2)$. When dealing with massive particles, the rapidity $y=1 / 2 \ln \left[\left(E+p_{z}\right) /\left(E-p_{z}\right)\right]$ is used, where $E$ is the energy and $p_{z}$ is the $z$-component of the momentum.
} 
signal and background distributions of the discriminating variables used in the final fit to the data. The multijet background is largely suppressed by the selection criteria and is estimated using data-driven techniques.

The signal templates in each STXS region were obtained from simulated $q \bar{q} \rightarrow W H$ and $q \bar{q} \rightarrow Z H$ events with zero or one additional jet, calculated at next-to-leading order (NLO), generated with the Powheg-Box v2 + GoSAm + MiNLO generators [25-28]. The contribution from loop-induced $g g \rightarrow Z H$ production was simulated at leading order (LO) using the Powheg-Box v2 generator [25]. Additional scale factors were applied to the $q \bar{q} \rightarrow V H$ processes as a function of the generated vector-boson transverse momentum $\left(p_{\mathrm{T}}^{V}\right)$ to account for electroweak $(\mathrm{EW})$ corrections at NLO. These factors were determined from the ratio between the $V H$ differential cross-sections computed with and without these corrections by the HAWK program [29, 30]. The mass of the Higgs boson was fixed at $125 \mathrm{GeV}$.

In the measurement of the $p p \rightarrow Z H$ cross-sections, the relative contributions of the $q \bar{q} \rightarrow Z H$ and $g g \rightarrow Z H$ processes are determined by the most accurate theoretical crosssection predictions currently available: next-to-next-to-leading order (NNLO) in QCD and NLO in EW [31-37] for $q \bar{q} \rightarrow Z H$, and next-to-leading order and next-to-leading logarithm $(\mathrm{NLO}+\mathrm{NLL})$ in QCD [38-42] for $g g \rightarrow Z H$.

\section{$3 \quad$ Event selection and categorisation}

The object reconstruction, event selection and classification into categories used for the measurements, are identical to those described in ref. [15]. The selection and the event categories are briefly summarised below.

Events are retained if they are consistent with one of the typical signatures of $V H$, $H \rightarrow b \bar{b}$ production and decay, with $Z \rightarrow \nu \bar{\nu}, W \rightarrow \ell \nu$ or $Z \rightarrow \ell \ell(\ell=e, \mu)$. Vector-boson decays into $\tau$-leptons are not targeted explicitly. However, they satisfy the selection criteria with reduced efficiency in the case of leptonic $\tau$-lepton decays.

In particular, events are kept if they contain at most two isolated electrons or muons, and two good-quality high- $p_{\mathrm{T}}(>45,20 \mathrm{GeV})$ jets with $|\eta|<2.5$ satisfying $b$-jet identification ('b-tagging') requirements (which have an average efficiency of $70 \%$ for jets containing $b$-hadrons that are produced in inclusive $t \bar{t}$ events [43]). The two $b$-jet candidates are used to reconstruct the Higgs boson candidate; their invariant mass is denoted by $m_{b b}$. Additional jets are required to have $p_{\mathrm{T}}>20 \mathrm{GeV}$ for $|\eta|<2.5$ or $p_{\mathrm{T}}>30 \mathrm{GeV}$ for $2.5<|\eta|<4.5$, and not be identified as $b$-jets.

Events with either zero, one or two isolated electrons or muons are classified as '0lepton', '1-lepton' or '2-lepton' events, respectively. The 0-lepton events and the 1-lepton events are required to have transverse momentum imbalance, as expected from the neutrinos from $Z \rightarrow \nu \bar{\nu}$ or $W \rightarrow \ell \nu$ decays; in the 2-lepton events, the leptons must have the same flavour (and opposite charge for events with muons) and an invariant mass close to the $Z$ boson mass.

Additional requirements are applied to suppress background from QCD production of multijet events in the 0-lepton and 1-lepton channels. To suppress the large $t \bar{t}$ background, 


\begin{tabular}{|c|c|c|c|c|c|}
\hline \multirow{3}{*}{ Channel } & \multicolumn{5}{|c|}{ Categories } \\
\hline & \multicolumn{2}{|c|}{$75 \mathrm{GeV}<p_{\mathrm{T}}^{V, \mathrm{r}}<150 \mathrm{GeV}$} & \multicolumn{3}{|c|}{$p_{\mathrm{T}}^{V, \mathrm{r}}>150 \mathrm{GeV}$} \\
\hline & 2 jets & $\geq 3$ jets & 2 jets & 3 jets & $\geq 3$ jets \\
\hline 0-lepton & - & - & $\mathrm{SR}$ & $\mathrm{SR}$ & - \\
\hline \multicolumn{6}{|l|}{ 1-lepton } \\
\hline$m_{b b} \geq 75 \mathrm{GeV}$ or $m_{\text {top }} \leq 225 \mathrm{GeV}$ & - & - & $\mathrm{SR}$ & $\mathrm{SR}$ & - \\
\hline$m_{b b}<75 \mathrm{GeV}$ and $m_{\text {top }}>225 \mathrm{GeV}$ & - & - & $\mathrm{CR}$ & $\mathrm{CR}$ & - \\
\hline \multicolumn{6}{|l|}{ 2-lepton } \\
\hline$e e$ and $\mu \mu$ channels & SR & SR & $\mathrm{SR}$ & - & $\mathrm{SR}$ \\
\hline$e \mu$ channel & $\mathrm{CR}$ & $\mathrm{CR}$ & $\mathrm{CR}$ & - & $\mathrm{CR}$ \\
\hline
\end{tabular}

Table 1. Summary of the reconstructed-event categories. Categories with relatively large fractions of the total expected signal yields are referred to as 'signal regions' (SR), while those with negligible expected signal yield, mainly designed to constrain some background processes, are called 'control regions' (CR). The quantity $m_{\mathrm{top}}$ is the reconstructed mass of a semileptonically decaying top-quark candidate in the 1-lepton channel. The calculation of $m_{\text {top }}$ uses the four-momenta of one of the two $b$-jet candidates, the lepton, and the hypothetical neutrino produced in the event. The neutrino four-momentum is derived using the $W$ boson mass constraint [15] and $m_{\text {top }}$ is then reconstructed from the combination of the $b$-jet candidate and the value of the neutrino longitudinal momentum that yields the smallest top-quark candidate mass. The $m_{\text {top }} \leq 225 \mathrm{GeV}$ requirement in the 1-lepton signal region is needed to maintain orthogonality with the $W+\mathrm{HF}$ control region.

events with four or more jets are discarded in the 0-lepton and 1-lepton channels. Finally, a requirement on the reconstructed transverse momentum $p_{\mathrm{T}}^{V, \mathrm{r}}$ of the vector boson $V$ is applied. It is computed, depending on the number, $N_{\text {lep }}$, of selected electrons and muons, as either the missing transverse momentum $E_{\mathrm{T}}^{\text {miss }}\left(N_{\text {lep }}=0\right)$, the magnitude of the vector sum of the missing transverse momentum and the lepton $p_{\mathrm{T}}\left(N_{\text {lep }}=1\right)$, or the dilepton $p_{\mathrm{T}}\left(N_{\text {lep }}=2\right)$. The minimum value of $p_{\mathrm{T}}^{V, \mathrm{r}}$ is $150 \mathrm{GeV}$ in the 0 - and 1-lepton channels, and $75 \mathrm{GeV}$ in the 2-lepton channel.

Events satisfying the previous criteria are classified into eight categories (also called signal regions in the following), shown in table 1, with different signal-to-background ratios. These categories are defined by the number of jets, $N_{\text {jet }}$ (including the two $b$-jet candidates), $N_{\text {lep }}$, and $p_{\mathrm{T}}^{V, \mathrm{r}}$. Additional categories (also called control regions in the following) containing events satisfying alternative selections are introduced to constrain some background processes such as $W$ boson production in association with jets containing heavy-flavour hadrons $(W+\mathrm{HF})$, or top-quark pair production. The signal contribution in such categories is expected to be negligible.

\section{Cross-section measurements}

The reduced $V H, V \rightarrow$ leptons stage-1 STXS regions used in this paper are summarised in table 2, which also indicates which reconstructed-event categories are most sensitive in 


\begin{tabular}{|c|c|c|c|c|c|}
\hline $\begin{array}{l}\text { Merged region } \\
\text { 3-POI scheme }\end{array}$ & $\begin{array}{l}\text { Merged region } \\
\text { 5-POI scheme }\end{array}$ & Stage 1 (modified) STXS region & \multicolumn{3}{|c|}{$\begin{array}{l}\text { Reconstructed-event categories } \\
\text { with largest sensitivity } \\
N_{\text {lep }} p_{\mathrm{T}}^{V, \mathrm{r}} \text { interval } N_{\text {jet }}\end{array}$} \\
\hline \multirow[t]{2}{*}{$W H, p_{\mathrm{T}}^{W}>150 \mathrm{GeV}$} & $W H, 150<p_{\mathrm{T}}^{W}<250 \mathrm{GeV}$ & $\begin{array}{l}q \bar{q} \rightarrow W H, 150<p_{\mathrm{T}}^{W}<250 \mathrm{GeV}, 0 \text {-jet } \\
q \bar{q} \rightarrow W H, 150<p_{\mathrm{T}}^{W}<250 \mathrm{GeV}, \geq 1 \text {-jet }\end{array}$ & \multirow[t]{2}{*}{1} & \multirow[t]{2}{*}{$>150 \mathrm{GeV}$} & \multirow[t]{2}{*}{2,3} \\
\hline & $W H, p_{\mathrm{T}}^{W}>250 \mathrm{GeV}$ & $q \bar{q} \rightarrow W H, p_{\mathrm{T}}^{W}>250 \mathrm{GeV}$ & & & \\
\hline$Z H, 75<p_{\mathrm{T}}^{Z}<150 \mathrm{GeV}$ & $Z H, 75<p_{\mathrm{T}}^{Z}<150 \mathrm{GeV}$ & $\begin{array}{l}q \bar{q} \rightarrow Z H, 75<p_{\mathrm{T}}^{Z}<150 \mathrm{GeV} \\
g g \rightarrow Z H, 75<p_{\mathrm{T}}^{Z}<150 \mathrm{GeV}\end{array}$ & 2 & $75-150 \mathrm{GeV}$ & $2, \geq 3$ \\
\hline \multirow[t]{2}{*}{$Z H, p_{\mathrm{T}}^{Z}>150 \mathrm{GeV}$} & $Z H, 150<p_{\mathrm{T}}^{Z}<250 \mathrm{GeV}$ & $\begin{aligned} q \bar{q} & \rightarrow Z H, 150<p_{\mathrm{T}}^{Z}<250 \mathrm{GeV}, 0 \text {-jet } \\
g g & \rightarrow Z H, 150<p_{\mathrm{T}}^{Z}<250 \mathrm{GeV}, 0 \text {-jet } \\
q \bar{q} & \rightarrow Z H, 150<p_{\mathrm{T}}^{Z}<250 \mathrm{GeV}, \geq 1 \text {-jet } \\
g g & \rightarrow Z H, 150<p_{\mathrm{T}}^{Z}<250 \mathrm{GeV}, \geq 1 \text {-jet }\end{aligned}$ & \multirow[t]{2}{*}{$\begin{array}{l}0 \\
2\end{array}$} & \multirow[t]{2}{*}{$\begin{array}{l}>150 \mathrm{GeV} \\
>150 \mathrm{GeV}\end{array}$} & \multirow[t]{2}{*}{$\begin{array}{l}2,3 \\
2, \geq 3\end{array}$} \\
\hline & $Z H, p_{\mathrm{T}}^{Z}>250 \mathrm{GeV}$ & $\begin{aligned} q \bar{q} & \rightarrow Z H, p_{\mathrm{T}}^{Z}>250 \mathrm{GeV} \\
g g & \rightarrow Z H, p_{\mathrm{T}}^{Z}>250 \mathrm{GeV}\end{aligned}$ & & & \\
\hline
\end{tabular}

Table 2. The 3-POI and 5-POI 'reduced stage-1' sets of merged regions used for the measurements, the corresponding kinematic regions of the stage-1 $V H$ simplified template cross-sections, and the reconstructed-event categories that are most sensitive in each merged region. The stage- 1 regions are modified (i) by splitting the two $Z H, p_{\mathrm{T}}^{Z}<150 \mathrm{GeV}$ regions (from $q \bar{q}$ and $g g$ ) into four regions, based on whether $p_{\mathrm{T}}^{Z}<75 \mathrm{GeV}$ or $75<p_{\mathrm{T}}^{Z}<150 \mathrm{GeV}$; (ii) by adding a $p_{\mathrm{T}}^{Z}<250 \mathrm{GeV}$ requirement to the $g g \rightarrow Z H, p_{\mathrm{T}}^{Z}>150 \mathrm{GeV}$ regions (with zero or at least one extra particle-level jet), and (iii) by adding a separate $g g \rightarrow Z H, p_{\mathrm{T}}^{Z}>250 \mathrm{GeV}$ region. The three regions $W H, p_{\mathrm{T}}^{W}<150 \mathrm{GeV}$, $q \bar{q} \rightarrow Z H, p_{\mathrm{T}}^{Z}<75 \mathrm{GeV}$ and $g g \rightarrow Z H, p_{\mathrm{T}}^{Z}<75 \mathrm{GeV}$, in which the current analysis is not sensitive and whose corresponding cross-sections are fixed to the SM prediction in the fit, are not shown.

each region. All leptonic decays of the weak gauge bosons (including $Z \rightarrow \tau \tau$ and $W \rightarrow \tau \nu$ ) are considered for the STXS definition.

Compared to the original stage-1 proposal presented in ref. [17], the following changes have been made for the reduced $V H, V \rightarrow$ leptons stage- 1 STXS regions of table 2:

- the $p_{\mathrm{T}}^{Z}<150 \mathrm{GeV}$ stage- 1 regions are split into two subregions, $p_{\mathrm{T}}^{Z}<75 \mathrm{GeV}$ and $75<p_{\mathrm{T}}^{Z}<150 \mathrm{GeV}$, to avoid theory uncertainties from extrapolations to a phase space not accessible to this measurement;

- an additional $g g \rightarrow Z H, p_{\mathrm{T}}^{Z}>250 \mathrm{GeV}$ region has been introduced, similarly to what is already done for $q \bar{q} \rightarrow Z H$.

These two changes lead to a total of 14 modified stage- 1 regions, which are then combined together in reduced stage- 1 regions, chosen to keep the total uncertainty in the measurements near or below $100 \%$, in the following way:

- the $q \bar{q} \rightarrow Z H$ and $g g \rightarrow Z H$ regions are merged. There are currently not enough data events to distinguish $q \bar{q} \rightarrow Z H$ from gluon-induced $Z H$ production despite their different kinematic properties;

- the $150<p_{\mathrm{T}}^{V}<250 \mathrm{GeV}$ regions with zero or at least one particle-level jet are merged. 


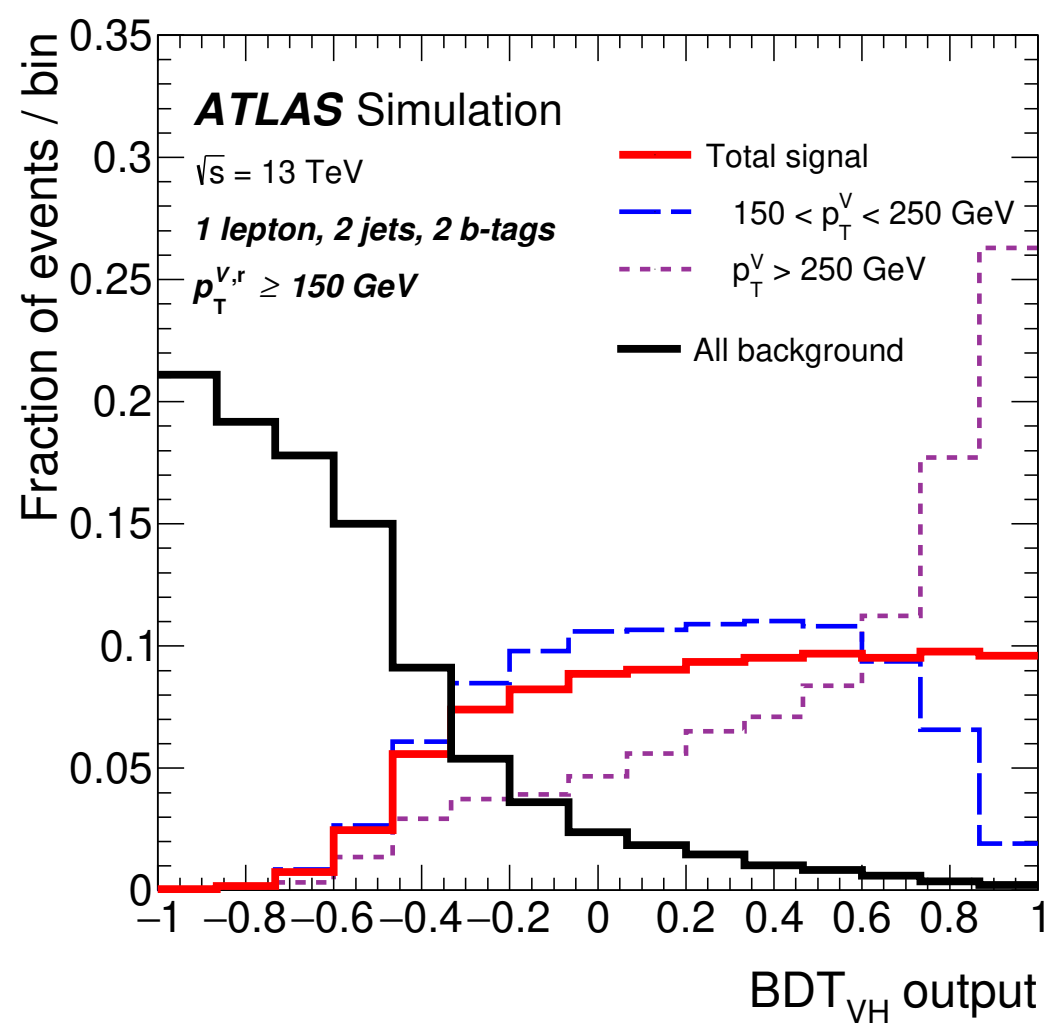

Figure 1. $\mathrm{BDT}_{V H}$ distributions for different $p_{\mathrm{T}}^{V} \mathrm{STXS}$ regions in the 1-lepton, 2-jet reconstructedevent category. Only regions contributing at least $10 \%$ of the expected signal yield in the reconstructed-event category are displayed. The distributions of the total signal and background are also shown. The $\mathrm{BDT}_{V H}$ distributions are scaled to the same (unit) area to highlight the shape differences.

Two sets of reduced stage- 1 regions are considered. In one, called the ' 5 -POI (parameters of interest)' scheme, five cross-sections, three for $Z H$ production $\left(75<p_{\mathrm{T}}^{Z}<150 \mathrm{GeV}\right.$, $150<p_{\mathrm{T}}^{Z}<250 \mathrm{GeV}$ and $\left.p_{\mathrm{T}}^{Z}>250 \mathrm{GeV}\right)$ and two for $W H$ production $\left(150<p_{\mathrm{T}}^{W}<250 \mathrm{GeV}\right.$ and $p_{\mathrm{T}}^{W}>250 \mathrm{GeV}$ ), are measured. In the other one, called the '3-POI' scheme, three cross-sections, two for $Z H\left(75<p_{\mathrm{T}}^{Z}<150 \mathrm{GeV}\right.$ and $\left.p_{\mathrm{T}}^{Z}>150 \mathrm{GeV}\right)$ and one for $W H$ $\left(p_{\mathrm{T}}^{W}>150 \mathrm{GeV}\right)$, are measured. The 5-POI scheme leads to measurements that have total uncertainties larger than those in the 3-POI scheme, but are more sensitive to enhancements at high $p_{\mathrm{T}}^{V}$ from potential anomalous interactions between the Higgs boson and the EW gauge bosons.

The reconstructed-event categories do not distinguish between events with generated $p_{\mathrm{T}}^{V}$ below or above $250 \mathrm{GeV}$. Discrimination between the two $p_{\mathrm{T}}^{V}$ regions $150-250 \mathrm{GeV}$ and $>250 \mathrm{GeV}$ is provided by the different shapes of the boosted-decision-tree discriminant $\left(\mathrm{BDT}_{V H}\right)$ used in the final fit to the data, as illustrated in figure 1 in the case of the 1-lepton, 2-jet category. This arises from the fact that the reconstructed $p_{\mathrm{T}}^{V, \mathrm{r}}$ is largely correlated with the $\mathrm{BDT}_{V H}$ output, for which it constitutes one of the most discriminating input variables together with $m_{b b}$ and the angular separation of the two $b$-jets. 
The product of the signal cross-section times the $H \rightarrow b \bar{b}$ branching ratio and the total leptonic decay branching ratio for $W$ or $Z$ bosons is determined in each of the reduced stage- 1 regions by a binned maximum-likelihood fit to the data. The cross-sections are not constrained to be positive in the fit. Signal and background templates of the discriminating variables, determined from the simulation or data control regions, are used to extract the signal and background yields. A simultaneous fit is performed to all the signal and control regions. Systematic uncertainties are included in the likelihood function as nuisance parameters.

The likelihood function is very similar to that described in ref. [15]. In particular, the same observables are used, namely $\mathrm{BDT}_{V H}$ in the signal regions and either the invariant mass $m_{b b}$ of the two $b$-jets or the event yield in the control regions. The treatment of the background and of its uncertainties is also unchanged. The only differences relative to the likelihood function in ref. [15] concern the treatment of the signal:

- Instead of a single signal shape (for $\mathrm{BDT}_{V H}$ or $m_{b b}$ ) or yield per category, multiple shapes or yields are introduced, one for each reduced stage-1 STXS region under study.

- Instead of a single parameter of interest, the inclusive signal strength, the fit has multiple parameters of interest, i.e. the cross-sections of the reduced stage- 1 regions, multiplied by the $H \rightarrow b \bar{b}$ and $V \rightarrow$ leptons branching ratios.

- Overall theoretical cross-section and branching ratio uncertainties, which affect the signal strength measurements but not the STXS measurements, are not included in the likelihood function.

The expected signal shapes of the discriminating variable distributions and the acceptance times efficiency (referred to as 'acceptance' in the following) in each reduced stage-1 region are determined from simulated samples of SM $V H, V \rightarrow$ leptons, $H \rightarrow b \bar{b}$ events. The acceptance of each reconstructed-event category for signal events from the different regions of the 5-POI reduced stage-1 scheme is shown in figure 2a. The fraction of signal events in each reconstructed-event category originating from the different regions in the same scheme is shown in figure $2 \mathrm{~b}$.

As shown in figure $2 \mathrm{a}$, the current analysis is not sensitive to $W H$ events with $p_{\mathrm{T}}^{W}<$ $150 \mathrm{GeV}$ and to $Z H$ events with $p_{\mathrm{T}}^{Z}<75 \mathrm{GeV}$, since their acceptance in each category is at the level of $0.1 \%$ or smaller. Therefore, in the fits the signal cross-section in these regions is constrained to the SM prediction, within the theoretical uncertainties. Since these regions contribute only marginally to the selected event sample, the impact on the final results is negligible. A cross-check in which the relative signal cross-section uncertainty for the $p_{\mathrm{T}}^{W}<150 \mathrm{GeV}$ and $p_{\mathrm{T}}^{Z}<75 \mathrm{GeV}$ regions is conservatively set to $70 \%$ of the prediction (i.e. about seven times the nominal uncertainty) leads to variations of the measured STXS below $1 \%$.

The sources of systematic uncertainty are identical to those described in ref. [15], except for those associated with the Higgs boson signal simulation, which are re-evaluated [44]. In this re-evaluation the uncertainties are separated into two groups: 


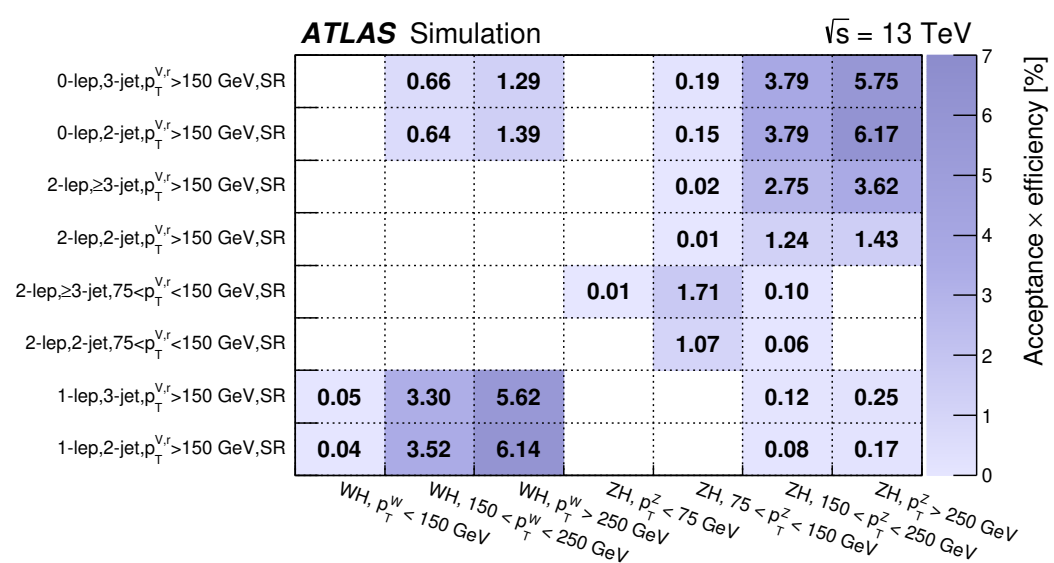

(a)

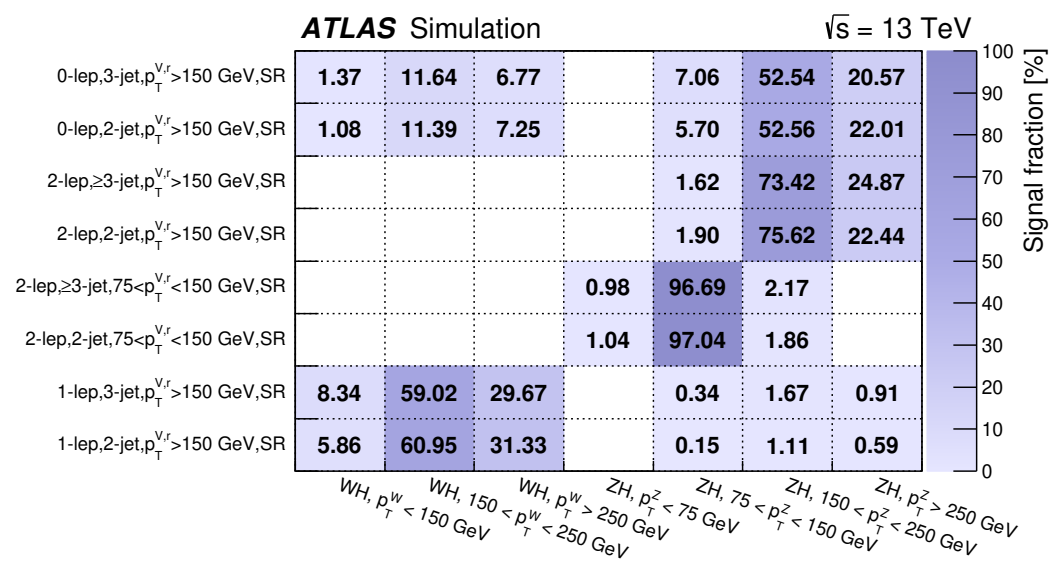

(b)

Figure 2. In the 5-POI reduced stage-1 scheme, (a) the acceptance (including the efficiency of the experimental selection) for $V H, V \rightarrow$ leptons, $H \rightarrow b \bar{b}$ events of each reconstructed-event category ( $y$-axis) for each STXS signal region ( $x$-axis), in percent; (b) the fraction of signal (in percent) from each STXS signal region ( $x$-axis) in every reconstructed-event category ( $y$-axis). Entries with acceptance times efficiency below $0.01 \%$ or signal fractions below $0.1 \%$ are not shown.

- uncertainties affecting signal modelling - i.e. acceptance and shape of kinematic distributions - in each of the three or five reduced stage-1 regions (hereafter referred to as theoretical modelling uncertainties), and

- uncertainties in the prediction of the production cross-section for each of these regions (hereafter referred to as theoretical cross-section uncertainties).

While theoretical modelling uncertainties enter the measurement of the STXS, theoretical cross-section uncertainties do not affect the results, but only the predictions with which they are compared. The consequent reduction of the impact of the theoretical uncertainties on the results with respect to the signal strength measurements is one of the main advantages of measuring STXS.

The two groups of systematic uncertainties are estimated for high-granularity STXS regions, and then merged into the reduced scheme under consideration. This approach 
makes it easy to compute the systematic uncertainties for merging schemes different from those presented here. The uncertainties are evaluated by dividing the phase space into five $p_{\mathrm{T}}^{V}$ regions (with the following lower edges: $0 \mathrm{GeV}, 75 \mathrm{GeV}, 150 \mathrm{GeV}, 250 \mathrm{GeV}$ and $400 \mathrm{GeV}$ ), and each $p_{\mathrm{T}}^{V}$ region into three bins depending on the number of particle-level jets (zero, one, or at least two), independently for the $q \bar{q} \rightarrow V H$ and $g g \rightarrow Z H$ processes. When two STXS regions are merged, their relative theoretical cross-section uncertainties lead to a modelling uncertainty. These uncertainties are evaluated as the remnant of the theoretical cross-section uncertainties for the high-granularity regions after the subtraction of the theoretical cross-section uncertainty for the merged region.

The high-granularity regions are used to calculate theoretical cross-section uncertainties for the missing higher-order terms in the QCD perturbative expansion and for the uncertainties induced by the choices of the parton distribution function (PDF) and $\alpha_{\mathrm{S}}$. Fourteen independent sources of uncertainties due to the missing higher-order terms lead to total uncertainties of $3 \%-4 \%$ for $q \bar{q} \rightarrow V H$ and $40 \%-50 \%$ for $g g \rightarrow Z H$ with $p_{\mathrm{T}}^{V}>$ $75 \mathrm{GeV}$ [44]. Thirty-one independent sources of PDF and $\alpha_{\mathrm{S}}$ uncertainties, each of them usually smaller than $1 \%$, lead to a total quadrature sum between $2 \%$ and $3 \%$ depending on the STXS region. The theoretical modelling uncertainties change the shapes of the reconstructed $p_{\mathrm{T}}^{V, \mathrm{r}}$ and $m_{b b}$ distributions in the same way as described in ref. [15]. Four independent sources for the QCD expansion and two independent sources for the PDF and $\alpha_{\mathrm{S}}$ choices are considered.

Systematic uncertainties in the signal acceptance and shape of the $p_{\mathrm{T}}^{V, \mathrm{r}}$ and $m_{b b}$ distributions due to the parton shower (PS) and underlying event (UE) models are estimated from the variations of acceptance and shapes of simulated events after changing the Pythia 8 PS parameters or after replacing Pythia 8 with HeRwig 7 for the PS and UE models [15]. The signal acceptance uncertainties due to the PS and UE models (five independent sources) are typically of the order of $1 \%(5 \%-15 \%)$ with a maximum of $10 \%$ (30\%) for the $q \bar{q} \rightarrow V H(g g \rightarrow Z H)$ production mode. Two independent nuisance parameters account for the systematic uncertainties induced by the PS and UE models in the $p_{\mathrm{T}}^{V, \mathrm{r}}$ and $m_{b b}$ distributions. In addition, a systematic uncertainty due to the EW corrections is parameterised as a change in shape of the $p_{\mathrm{T}}^{V}$ distributions for the $q \bar{q} \rightarrow V H$ processes [15].

\section{Results}

The measured reduced stage- $1 V H$ cross-sections times the $H \rightarrow b \bar{b}$ and $V \rightarrow$ leptons branching ratios, $\sigma \times B$, in the 5-POI and 3-POI schemes, together with the SM predictions, are summarised in table 3 . The results of the 5 -POI scheme are also illustrated in figure 3 . The SM predictions are shown together with the theoretical cross-section uncertainty for the merged regions computed as described in the previous section. The measurements are in agreement with the SM predictions.

The cross-sections measured in the $p_{\mathrm{T}}^{V}>150 \mathrm{GeV}$ intervals are not equal to the sum of those measured for $150<p_{\mathrm{T}}^{V}<250 \mathrm{GeV}$ and $p_{\mathrm{T}}^{V}>250 \mathrm{GeV}$. This is because the signal template for $p_{\mathrm{T}}^{V}>150 \mathrm{GeV}$ in the 3-POI fit is computed from the sum of the templates of the two regions assuming that the ratio of yields in those regions is that predicted 


\begin{tabular}{|c|c|c|c|c|c|c|c|c|c|c|c|c|c|c|}
\hline \multirow{2}{*}{$\begin{array}{l}\text { Measurement region } \\
\left(\left|y_{H}\right|<2.5, H \rightarrow b \bar{b}\right)\end{array}$} & \multirow{2}{*}{\multicolumn{3}{|c|}{$\begin{array}{l}\text { SM prediction } \\
\qquad \mathrm{fb}]\end{array}$}} & \multirow{2}{*}{\multicolumn{3}{|c|}{$\begin{array}{l}\text { Result } \\
{[\mathrm{fb}]}\end{array}$}} & \multirow{2}{*}{\multicolumn{2}{|c|}{$\begin{array}{l}\text { Stat. unc. } \\
\qquad[\mathrm{fb}]\end{array}$}} & \multicolumn{6}{|c|}{ Syst. unc. [fb] } \\
\hline & & & & & & & & & & sig. & & bkg. & & \\
\hline \multicolumn{15}{|c|}{ 5-POI scheme } \\
\hline$W \rightarrow \ell \nu ; 150<p_{\mathrm{T}}^{V}<250 \mathrm{GeV}$ & 24.0 & \pm & 1.1 & 20 & \pm & 25 & \pm & 17 & \pm & 2 & \pm & & & 9 \\
\hline$W \rightarrow \ell \nu ; p_{\mathrm{T}}^{V}>250 \mathrm{GeV}$ & 7.1 & \pm & 0.3 & 8.8 & \pm & 5.2 & \pm & 4.4 & \pm & 0.5 & \pm & 2.5 & & 0.9 \\
\hline$Z \rightarrow \ell \ell, \nu \nu ; 75<p_{\mathrm{T}}^{V}<150 \mathrm{GeV}$ & 50.6 & \pm & 4.1 & 81 & \pm & 45 & \pm & 35 & \pm & 10 & \pm & 21 & \pm & 19 \\
\hline$Z \rightarrow \ell \ell, \nu \nu ; 150<p_{\mathrm{T}}^{V}<250 \mathrm{GeV}$ & 18.8 & \pm & 2.4 & 14 & & 13 & \pm & 11 & \pm & 1 & \pm & 6 & \pm & 3 \\
\hline$Z \rightarrow \ell \ell, \nu \nu ; p_{\mathrm{T}}^{V}>250 \mathrm{GeV}$ & 4.9 & \pm & 0.5 & 8.5 & \pm & 4.0 & \pm & 3.7 & \pm & 0.8 & \pm & 1.2 & \pm & 0.6 \\
\hline \multicolumn{15}{|c|}{ 3-POI scheme } \\
\hline$W \rightarrow \ell \nu ; p_{\mathrm{T}}^{V}>150 \mathrm{GeV}$ & 31.1 & \pm & 1.4 & 35 & \pm & 14 & \pm & 9 & \pm & 2 & \pm & 9 & \pm & 4 \\
\hline$Z \rightarrow \ell \ell, \nu \nu ; 75<p_{\mathrm{T}}^{V}<150 \mathrm{GeV}$ & 50.6 & \pm & 4.1 & 81 & \pm & 45 & \pm & 35 & \pm & 10 & \pm & 21 & \pm & 19 \\
\hline$Z \rightarrow \ell \ell, \nu \nu ; p_{\mathrm{T}}^{V}>150 \mathrm{GeV}$ & 23.7 & \pm & 3.0 & 28.4 & \pm & 8.1 & \pm & 6.4 & \pm & 2.4 & \pm & 3.6 & \pm & 2.3 \\
\hline
\end{tabular}

Table 3. Best-fit values and uncertainties for the $V H, V \rightarrow$ leptons reduced stage-1 simplified template cross-sections times the $H \rightarrow b \bar{b}$ branching ratio, in the 5-POI (top five rows) and 3-POI (bottom three rows) schemes. The SM predictions for each region, computed using the inclusive cross-section calculations and the simulated event samples described in section 2, are also shown. The contributions to the total uncertainty in the measurements from statistical (Stat. unc.) or systematic uncertainties (Syst. unc.) in the signal modelling (Th. sig.), background modelling (Th. bkg.), and in experimental performance (Exp.) are given separately. The total systematic uncertainty, equal to the difference in quadrature between the total uncertainty and the statistical uncertainty, differs from the sum in quadrature of the Th. Sig., Th. Bkg., and Exp. systematic uncertainties due to correlations. All leptonic decays of the $V$ bosons (including those to $\tau$-leptons, $\ell=e, \mu, \tau)$ are considered.

by the SM, while in the 5-POI fit the normalisations of the two templates are floated independently.

The cross-sections are measured with relative uncertainties varying between $50 \%$ and $125 \%$ in the 5 -POI case, and between $29 \%$ and $56 \%$ for the 3 -POI. The largest uncertainties are statistical, except for the $W H$ cross-sections with $p_{\mathrm{T}}^{W}>150 \mathrm{GeV}$ in the 3 -POI case and with $150<p_{\mathrm{T}}^{W}<250 \mathrm{GeV}$ in the 5 -POI case. In the 5 -POI case, an anti-correlation of the order of $40 \%-60 \%$ is observed between the cross-sections in the ranges $p_{\mathrm{T}}^{V}>250 \mathrm{GeV}$ and $150<p_{\mathrm{T}}^{V}<250 \mathrm{GeV}$, which are measured with the same reconstructed-event categories.

The dominant systematic uncertainties are due to the limited number of simulated background events and the theoretical modelling of the background processes. The uncertainties due to the theoretical modelling of the $V H$ signal are small, with relative values ranging between $6 \%$ and $12 \%$. The uncertainties in the predictions are 2-3 times larger for $Z H$ than for $W H$ in the same $p_{\mathrm{T}}^{V}$ interval due to the limited precision of the theoretical calculations of the $g g \rightarrow Z H$ process. 


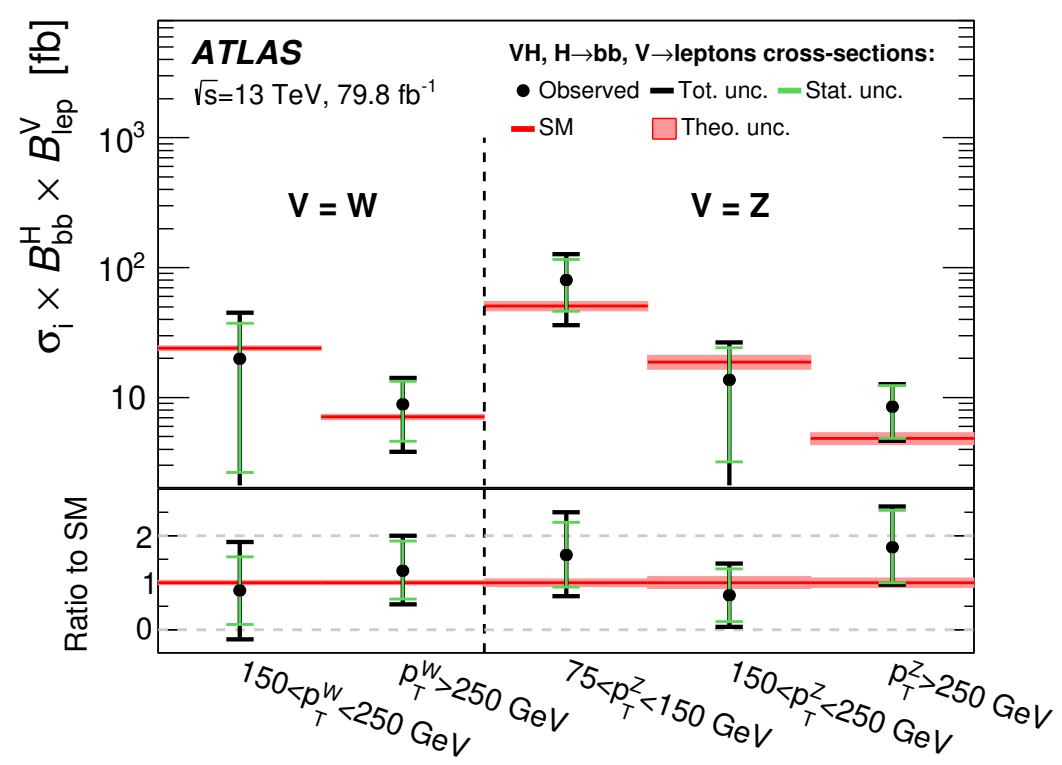

Figure 3. Measured $V H, V \rightarrow$ leptons reduced stage-1 simplified template cross-sections times the $H \rightarrow b \bar{b}$ branching ratio.

\section{Constraints on anomalous Higgs boson interactions}

The strength and tensor structure of the Higgs boson interactions are investigated using an effective Lagrangian approach [22]. Extra terms of the form $c_{i}^{(D)} \mathcal{O}_{i}^{(D)} / \Lambda^{D-4}$, where $\Lambda$ is the energy scale of the new interactions, $O_{i}^{(D)}$ are dimension- $D$ operators, and $c_{i}^{(D)}$ are numerical coefficients, are added to the SM Lagrangian to obtain an effective Lagrangian inspired by that in ref. [45]. Only dimension $D=6$ operators are considered in this study, since dimension $D=5$ operators violate lepton or baryon number, while dimension $D>6$ operators are further suppressed by powers of $\Lambda$.

The results presented in this paper focus on the coefficients of the operators in the 'Strongly Interacting Light Higgs' formulation [46]. This formalism is defined as the effective theory of a strongly interacting sector in which a light composite Higgs boson arises as a pseudo Goldstone boson, and is responsible for EW symmetry breaking. Among such operators, four directly affect the $V H$ cross-sections because they introduce new Higgs boson interactions with $W$ bosons $\left(\mathcal{O}_{H W}, \mathcal{O}_{W}\right)$ and $Z$ bosons (all four operators):

- $\mathcal{O}_{H W}=i\left(D^{\mu} H\right)^{\dagger} \sigma^{a}\left(D^{\nu} H\right) W_{\mu \nu}^{a}$,

- $\mathcal{O}_{H B}=i\left(D^{\mu} H\right)^{\dagger}\left(D^{\nu} H\right) B_{\mu \nu}$,

- $\mathcal{O}_{W}=\frac{i}{2}\left(H^{\dagger} \sigma^{a} \stackrel{\leftrightarrow}{D^{\mu}} H\right) D^{\nu} W_{\mu \nu}^{a}$

- $\mathcal{O}_{B}=\frac{i}{2}\left(H^{\dagger} \stackrel{\leftrightarrow}{D^{\mu}} H\right) \partial^{\nu} B_{\mu \nu}$

The corresponding $C P$-odd operators $\tilde{\mathcal{O}}_{H W}, \tilde{\mathcal{O}}_{H B}, \tilde{\mathcal{O}}_{W}$, and $\tilde{\mathcal{O}}_{B}$, are not considered. 
Modifications of the $g g \rightarrow Z H$ production cross-section are only introduced by either higher-dimension $(D \geq 8)$ operators or corrections that are formally at NNLO in QCD, and are not included in this study, in which the expected $g g \rightarrow Z H$ contribution is kept fixed to the SM prediction.

The operator $\mathcal{O}_{d}=y_{d}|H|^{2} \bar{Q}_{\mathrm{L}} H d_{\mathrm{R}}$ (plus Hermitian conjugate) with Yukawa coupling strength $y_{d}$, which modifies the coupling between the Higgs boson and down-type quarks, induces variations of the partial width $\Gamma_{H}^{b b}$ and of the total Higgs boson width $\Gamma_{H}$, and therefore of the $H \rightarrow b \bar{b}$ branching ratio. This operator affects the measured cross-sections in the same way in each region.

Constraints are set on the coefficients of the five $\mathcal{O}_{W}, \mathcal{O}_{B}, \mathcal{O}_{H W}, \mathcal{O}_{H B}$ and $\mathcal{O}_{d}$ operators in the 'Higgs Effective Lagrangian' (HEL) implementation [47], using the known relations between such coefficients and the stage-1 STXS based on leading-order predictions [48]. Such relations include interference terms between the SM and non-SM amplitudes that are linear in the coefficients and of order $1 / \Lambda^{2}$, and the SM-independent contributions that are quadratic in the coefficients and of order $1 / \Lambda^{4}$. In the HEL implementation, the coefficients $c_{i}$ of interest are recast into the following dimensionless coefficients:

$$
\bar{c}_{H W}=\frac{m_{W}^{2}}{g} \frac{c_{H W}}{\Lambda^{2}}, \quad \bar{c}_{H B}=\frac{m_{W}^{2}}{g^{\prime}} \frac{c_{H B}}{\Lambda^{2}}, \quad \bar{c}_{W}=\frac{m_{W}^{2}}{g} \frac{c_{W}}{\Lambda^{2}}, \quad \bar{c}_{B}=\frac{m_{W}^{2}}{g^{\prime}} \frac{c_{B}}{\Lambda^{2}}, \quad \bar{c}_{d}=v^{2} \frac{c_{d}}{\Lambda^{2}},
$$

where $g$ and $g^{\prime}$ are the $\mathrm{SU}(2)$ and $\mathrm{U}(1)$ SM gauge couplings, and $v$ is the vacuum expectation value of the Higgs boson field. These dimensionless coefficients are equal to zero in the SM.

The sum $\bar{c}_{W}+\bar{c}_{B}$ is strongly constrained by precision EW data [49] and is thus assumed here to be zero, and constraints are set on $\bar{c}_{H W}, \bar{c}_{H B}, \bar{c}_{W}-\bar{c}_{B}$ and $\bar{c}_{d}$. The relations between the HEL coefficients and the reduced STXS measured in this paper are obtained by averaging the relations for the regions that are merged with weights proportional to their respective cross-sections.

Simultaneous maximum-likelihood fits to the five STXS measured in the 5-POI scheme are performed to determine $\bar{c}_{H W}, \bar{c}_{H B}, \bar{c}_{W}-\bar{c}_{B}$ and $\bar{c}_{d}$. Due to the large sensitivity to the Higgs boson anomalous couplings to vector bosons provided by the $p_{\mathrm{T}}^{V}>250 \mathrm{GeV}$ crosssections, the 5-POI results place tighter constraints on these coefficients (e.g. approximately a factor two for $\bar{c}_{H W}$ ) than do the 3-POI results. For this reason, constraints obtained with the 3-POI results are not shown here.

In each fit, all coefficients but one are assumed to vanish, and $68 \%$ and $95 \%$ confidence level (CL) one-dimensional intervals are inferred for the remaining coefficient. The negativelog-likelihood one-dimensional projections are shown in figure 4, and the $68 \%$ and $95 \% \mathrm{CL}$ intervals for $\bar{c}_{H W}, \bar{c}_{H B}, \bar{c}_{W}-\bar{c}_{B}$ and $\bar{c}_{d}$ are summarised in table 4 . The parameters $\bar{c}_{H W}$ and $\bar{c}_{W}-\bar{c}_{B}$ are constrained at $95 \%$ CL to be no more than a few percent, while the constraint on $\bar{c}_{H B}$ is about five times worse, and the constraint on $\bar{c}_{d}$ is of order unity. For comparison, table 4 also shows the $68 \%$ and $95 \%$ CL intervals for the dimensionless coefficients when the SM-independent contributions, which are of the same order $\left(1 / \Lambda^{4}\right)$ as the dimension- 8 operators that are neglected, are not considered. The constraints are typically $50 \%$ stronger than when the SM-independent contributions are not neglected. 


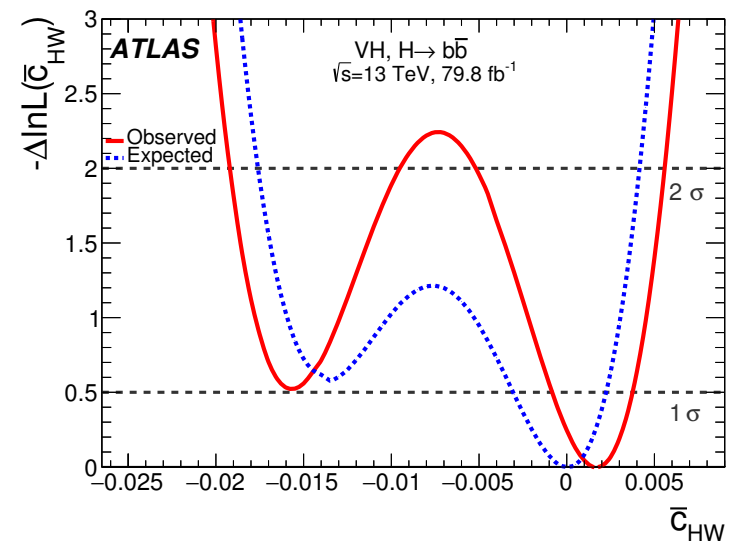

(a)

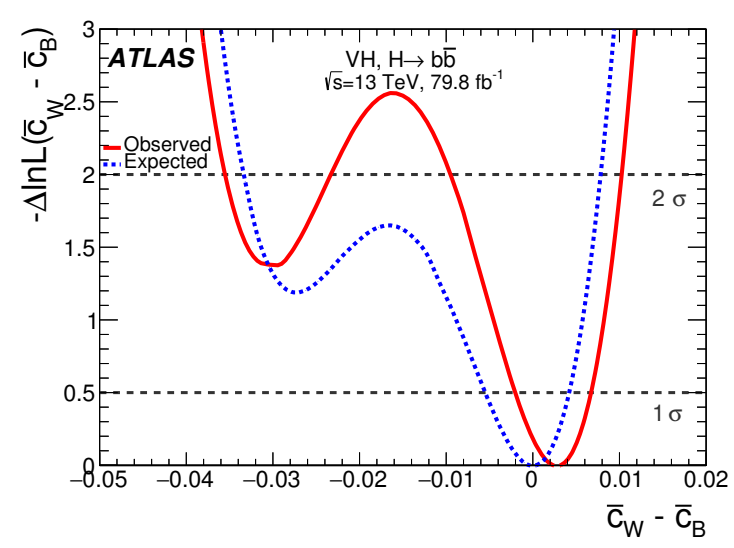

(c)

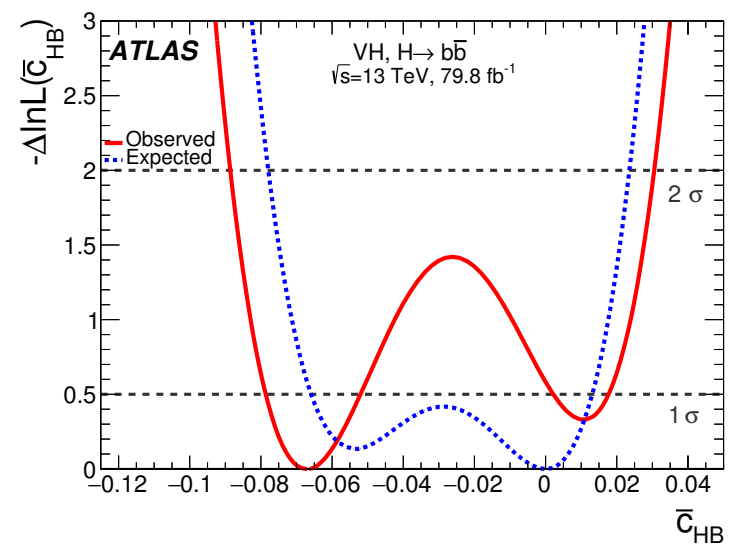

(b)

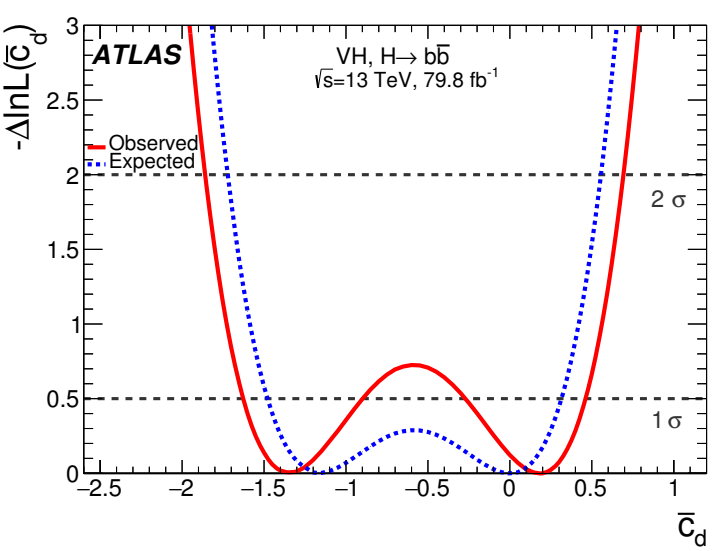

(d)

Figure 4. The observed (solid) and expected (dotted) profiled negative-log-likelihood functions for the one-dimensional fits to constrain the coefficients (a) $\bar{c}_{H W}$, (b) $\bar{c}_{H B}$, (c) $\bar{c}_{W}-\bar{c}_{B}$ and (d) $\bar{c}_{d}$ of an effective Lagrangian (described in the text), when the other coefficients are assumed to vanish.

\section{Conclusion}

Using $79.8 \mathrm{fb}^{-1}$ of $\sqrt{s}=13 \mathrm{TeV}$ proton-proton collisions collected by the ATLAS detector at the LHC, the cross-sections for the associated production of a Higgs boson decaying into bottom-quark pairs and an electroweak gauge boson $W$ or $Z$ decaying into leptons are measured as functions of the vector-boson transverse momentum $p_{\mathrm{T}}^{V}$. The cross-sections are measured for Higgs bosons in a fiducial volume with rapidity $\left|y_{H}\right|<2.5$, in the 'simplified template cross-section' framework.

The measurements are performed for two different choices of the number of $p_{\mathrm{T}}^{V}$ intervals. The results have relative uncertainties varying between $50 \%$ and $125 \%$ in one case, and between $29 \%$ and $56 \%$ in the other. The measurements are in agreement with the Standard Model predictions, even in high $p_{\mathrm{T}}^{V}(>250 \mathrm{GeV})$ regions that are most sensitive to enhancements from potential anomalous interactions between the Higgs boson and the electroweak gauge bosons. 


\begin{tabular}{|c|c|c|}
\hline Coefficient & Expected interval & Observed interval \\
\hline \multicolumn{3}{|c|}{ Results at $68 \%$ confidence level } \\
\hline $\bar{c}_{H W}$ & {$[-0.003,0.002]$} & {$[-0.001,0.004]$} \\
\hline (interference only & {$[-0.002,0.003]$} & $[-0.001,0.005])$ \\
\hline $\bar{c}_{H B}$ & {$[-0.066,0.013]$} & {$[-0.078,-0.055] \cup[0.005,0.019]$} \\
\hline (interference only & {$[-0.016,0.016]$} & $[-0.005,0.030])$ \\
\hline $\bar{c}_{W}-\bar{c}_{B}$ & {$[-0.006,0.005]$} & {$[-0.002,0.007]$} \\
\hline (interference only & {$[-0.005,0.005]$} & $[-0.002,0.008])$ \\
\hline $\bar{c}_{d}$ & {$[-1.5,0.3]$} & {$[-1.6,-0.9] \cup[-0.3,0.4]$} \\
\hline (interference only & {$[-0.4,0.4]$} & $[-0.2,0.7])$ \\
\hline \multicolumn{3}{|c|}{ Results at $95 \%$ confidence level } \\
\hline $\bar{c}_{H W}$ & {$[-0.018,0.004]$} & {$[-0.019,-0.010] \cup[-0.005,0.006]$} \\
\hline (interference only & {$[-0.005,0.005]$} & $[-0.003,0.008])$ \\
\hline $\bar{c}_{H B}$ & {$[-0.078,0.024]$} & {$[-0.090,0.032]$} \\
\hline (interference only & {$[-0.033,0.033]$} & $[-0.022,0.049])$ \\
\hline $\bar{c}_{W}-\bar{c}_{B}$ & {$[-0.034,0.008]$} & {$[-0.036,-0.024] \cup[-0.009,0.010]$} \\
\hline (interference only & {$[-0.009,0.010]$} & $[-0.006,0.014])$ \\
\hline $\bar{c}_{d}$ & {$[-1.7,0.5]$} & {$[-1.9,0.7]$} \\
\hline (interference only & {$[-0.8,0.8]$} & $[-0.6,1.1])$ \\
\hline
\end{tabular}

Table 4. The expected and observed $68 \%$ CL (four top rows) and 95\% CL (four bottom rows) intervals for the effective Lagrangian coefficients $\bar{c}_{H W}, \bar{c}_{H B}, \bar{c}_{W}-\bar{c}_{B}$ and $\bar{c}_{d}$ when the other coefficients are assumed to vanish. Each row is composed of two sub-rows: the first one uses the interference between SM and non-SM amplitudes and the SM-independent contributions, while the second sub-row uses only the interference between SM and non-SM amplitudes.

One-dimensional limits on four linear combinations of the coefficients of effective Lagrangian operators affecting the Higgs boson couplings to the electroweak gauge bosons and to down-type quarks have also been set. For two of these parameters the constraint has a precision of a few percent.

\section{Acknowledgments}

We thank CERN for the very successful operation of the LHC, as well as the support staff from our institutions without whom ATLAS could not be operated efficiently.

We acknowledge the support of ANPCyT, Argentina; YerPhI, Armenia; ARC, Australia; BMWFW and FWF, Austria; ANAS, Azerbaijan; SSTC, Belarus; CNPq and FAPESP, Brazil; NSERC, NRC and CFI, Canada; CERN; CONICYT, Chile; CAS, MOST and NSFC, China; COLCIENCIAS, Colombia; MSMT CR, MPO CR and VSC CR, Czech Republic; DNRF and DNSRC, Denmark; IN2P3-CNRS, CEA-DRF/IRFU, France; 
SRNSFG, Georgia; BMBF, HGF, and MPG, Germany; GSRT, Greece; RGC, Hong Kong SAR, China; ISF and Benoziyo Center, Israel; INFN, Italy; MEXT and JSPS, Japan; CNRST, Morocco; NWO, Netherlands; RCN, Norway; MNiSW and NCN, Poland; FCT, Portugal; MNE/IFA, Romania; MES of Russia and NRC KI, Russian Federation; JINR; MESTD, Serbia; MSSR, Slovakia; ARRS and MIZŠ, Slovenia; DST/NRF, South Africa; MINECO, Spain; SRC and Wallenberg Foundation, Sweden; SERI, SNSF and Cantons of Bern and Geneva, Switzerland; MOST, Taiwan; TAEK, Turkey; STFC, United Kingdom; DOE and NSF, United States of America. In addition, individual groups and members have received support from BCKDF, CANARIE, CRC and Compute Canada, Canada; COST, ERC, ERDF, Horizon 2020, and Marie Skłodowska-Curie Actions, European Union; Investissements d' Avenir Labex and Idex, ANR, France; DFG and AvH Foundation, Germany; Herakleitos, Thales and Aristeia programmes co-financed by EU-ESF and the Greek NSRF, Greece; BSF-NSF and GIF, Israel; CERCA Programme Generalitat de Catalunya, Spain; The Royal Society and Leverhulme Trust, United Kingdom.

The crucial computing support from all WLCG partners is acknowledged gratefully, in particular from CERN, the ATLAS Tier-1 facilities at TRIUMF (Canada), NDGF (Denmark, Norway, Sweden), CC-IN2P3 (France), KIT/GridKA (Germany), INFN-CNAF (Italy), NL-T1 (Netherlands), PIC (Spain), ASGC (Taiwan), RAL (U.K.) and BNL (U.S.A.), the Tier-2 facilities worldwide and large non-WLCG resource providers. Major contributors of computing resources are listed in ref. [50].

Open Access. This article is distributed under the terms of the Creative Commons Attribution License (CC-BY 4.0), which permits any use, distribution and reproduction in any medium, provided the original author(s) and source are credited.

\section{References}

[1] F. Englert and R. Brout, Broken Symmetry and the Mass of Gauge Vector Mesons, Phys. Rev. Lett. 13 (1964) 321 [INSPIRE].

[2] P.W. Higgs, Broken symmetries, massless particles and gauge fields, Phys. Lett. 12 (1964) 132 [INSPIRE].

[3] P.W. Higgs, Broken Symmetries and the Masses of Gauge Bosons, Phys. Rev. Lett. 13 (1964) 508 [INSPIRE].

[4] G.S. Guralnik, C.R. Hagen and T.W.B. Kibble, Global Conservation Laws and Massless Particles, Phys. Rev. Lett. 13 (1964) 585 [inSPIRE].

[5] ATLAS collaboration, Observation of a new particle in the search for the Standard Model Higgs boson with the ATLAS detector at the LHC, Phys. Lett. B 716 (2012) 1 [arXiv: 1207.7214] [INSPIRE].

[6] CMS collaboration, Observation of a new boson at a mass of $125 \mathrm{GeV}$ with the CMS experiment at the LHC, Phys. Lett. B 716 (2012) 30 [arXiv:1207.7235] [INSPIRE].

[7] ATLAS and CMS collaborations, Combined Measurement of the Higgs Boson Mass in pp Collisions at $\sqrt{s}=7$ and 8 TeV with the ATLAS and CMS Experiments, Phys. Rev. Lett. 114 (2015) 191803 [arXiv:1503.07589] [INSPIRE]. 
[8] ATLAS collaboration, Measurement of the Higgs boson mass in the $H \rightarrow Z Z^{*} \rightarrow 4 \ell$ and $H \rightarrow \gamma \gamma$ channels with $\sqrt{s}=13$ TeV pp collisions using the ATLAS detector, Phys. Lett. B 784 (2018) 345 [arXiv: 1806.00242] [INSPIRE].

[9] CMS collaboration, Measurements of properties of the Higgs boson decaying into the four-lepton final state in pp collisions at $\sqrt{s}=13 \mathrm{TeV}$, JHEP 11 (2017) 047 [arXiv: 1706.09936] [INSPIRE].

[10] ATLAS collaboration, Study of the spin and parity of the Higgs boson in diboson decays with the ATLAS detector, Eur. Phys. J. C 75 (2015) 476 [Erratum ibid. C 76 (2016) 152] [arXiv: 1506.05669] [INSPIRE].

[11] CMS collaboration, Constraints on the spin-parity and anomalous HVV couplings of the Higgs boson in proton collisions at 7 and 8 TeV, Phys. Rev. D 92 (2015) 012004 [arXiv: 1411.3441] [INSPIRE].

[12] ATLAS and CMS collaborations, Measurements of the Higgs boson production and decay rates and constraints on its couplings from a combined ATLAS and CMS analysis of the LHC pp collision data at $\sqrt{s}=7$ and $8 \mathrm{TeV}$, JHEP 08 (2016) 045 [arXiv:1606.02266] [INSPIRE].

[13] CMS collaboration, Observation of $t \bar{t} H$ production, Phys. Rev. Lett. 120 (2018) 231801 [arXiv: 1804.02610] [INSPIRE].

[14] ATLAS collaboration, Observation of Higgs boson production in association with a top quark pair at the LHC with the ATLAS detector, Phys. Lett. B 784 (2018) 173 [arXiv: 1806.00425] [INSPIRE].

[15] ATLAS collaboration, Observation of $H \rightarrow b \bar{b}$ decays and $V H$ production with the ATLAS detector, Phys. Lett. B 786 (2018) 59 [arXiv: 1808.08238] [INSPIRE].

[16] CMS collaboration, Observation of Higgs boson decay to bottom quarks, Phys. Rev. Lett. 121 (2018) 121801 [arXiv: 1808.08242] [INSPIRE].

[17] LHC Higgs Cross Section Working Group, Handbook of LHC Higgs Cross Sections: 4. Deciphering the Nature of the Higgs Sector, CERN Publishing (2016).

[18] J.R. Andersen et al., Les Houches 2015: Physics at TeV Colliders Standard Model Working Group Report, in proceedings of the 9th Les Houches Workshop on Physics at TeV Colliders (PhysTeV 2015), Les Houches, France, 1-19 June 2015, arXiv:1605.04692 [InSPIRE].

[19] M. Cacciari, G.P. Salam and G. Soyez, The anti- $k_{t}$ jet clustering algorithm, JHEP 04 (2008) 063 [arXiv: 0802.1189] [INSPIRE].

[20] ATLAS collaboration, Measurements of Higgs boson properties in the diphoton decay channel with $36 \mathrm{fb}^{-1}$ of pp collision data at $\sqrt{s}=13 \mathrm{TeV}$ with the ATLAS detector, Phys. Rev. D 98 (2018) 052005 [arXiv: 1802.04146] [INSPIRE].

[21] ATLAS collaboration, Measurement of the Higgs boson coupling properties in the $H \rightarrow Z Z^{*} \rightarrow 4 \ell$ decay channel at $\sqrt{s}=13 \mathrm{TeV}$ with the ATLAS detector, JHEP 03 (2018) 095 [arXiv: 1712. 02304] [INSPIRE].

[22] ATLAS collaboration, Constraints on an effective Lagrangian from the combined $H \rightarrow Z Z^{*} \rightarrow 4 \ell$ and $H \rightarrow \gamma \gamma$ channels using $36.1 \mathrm{fb}^{-1}$ of $\sqrt{s}=13 \mathrm{TeV}$ pp collision data collected with the ATLAS detector, ATL-PHYS-PUB-2017-018 (2017).

[23] ATLAS collaboration, The ATLAS Experiment at the CERN Large Hadron Collider, 2008 JINST 3 S08003 [INSPIRE]. 
[24] ATLAS IBL collaboration, Production and Integration of the ATLAS Insertable B-Layer, 2018 JINST 13 T05008 [arXiv:1803.00844] [INSPIRE].

[25] S. Alioli, P. Nason, C. Oleari and E. Re, A general framework for implementing NLO calculations in shower Monte Carlo programs: the POWHEG BOX, JHEP 06 (2010) 043 [arXiv: 1002.2581] [INSPIRE].

[26] G. Cullen et al., Automated One-Loop Calculations with GoSam, Eur. Phys. J. C 72 (2012) 1889 [arXiv: 1111.2034] [INSPIRE].

[27] K. Hamilton, P. Nason and G. Zanderighi, MINLO: Multi-Scale Improved NLO, JHEP 10 (2012) 155 [arXiv:1206.3572] [INSPIRE].

[28] G. Luisoni, P. Nason, C. Oleari and F. Tramontano, $H W^{ \pm} / H Z+0$ and 1 jet at $N L O$ with the POWHEG BOX interfaced to GoSam and their merging within MiNLO, JHEP 10 (2013) 083 [arXiv: 1306.2542] [INSPIRE].

[29] A. Denner, S. Dittmaier, S. Kallweit and A. Mück, Electroweak corrections to Higgs-strahlung off $W / Z$ bosons at the Tevatron and the LHC with HAWK, JHEP 03 (2012) 075 [arXiv: 1112.5142] [INSPIRE].

[30] A. Denner, S. Dittmaier, S. Kallweit and A. Mück, HAWK 2.0: A Monte Carlo program for Higgs production in vector-boson fusion and Higgs strahlung at hadron colliders, Comput. Phys. Commun. 195 (2015) 161 [arXiv:1412.5390] [INSPIRE].

[31] M.L. Ciccolini, S. Dittmaier and M. Krämer, Electroweak radiative corrections to associated WH and ZH production at hadron colliders, Phys. Rev. D 68 (2003) 073003 [hep-ph/0306234] [INSPIRE].

[32] O. Brein, A. Djouadi and R. Harlander, NNLO QCD corrections to the Higgs-strahlung processes at hadron colliders, Phys. Lett. B 579 (2004) 149 [hep-ph/0307206] [INSPIRE].

[33] G. Ferrera, M. Grazzini and F. Tramontano, Associated WH production at hadron colliders: a fully exclusive QCD calculation at NNLO, Phys. Rev. Lett. 107 (2011) 152003 [arXiv: 1107.1164] [INSPIRE].

[34] O. Brein, R. Harlander, M. Wiesemann and T. Zirke, Top-Quark Mediated Effects in Hadronic Higgs-Strahlung, Eur. Phys. J. C 72 (2012) 1868 [arXiv:1111.0761] [INSPIRE].

[35] G. Ferrera, M. Grazzini and F. Tramontano, Higher-order QCD effects for associated WH production and decay at the LHC, JHEP 04 (2014) 039 [arXiv: 1312.1669] [INSPIRE].

[36] G. Ferrera, M. Grazzini and F. Tramontano, Associated ZH production at hadron colliders: the fully differential NNLO QCD calculation, Phys. Lett. B $\mathbf{7 4 0 ~ ( 2 0 1 5 ) ~} 51$ [arXiv: 1407.4747] [INSPIRE].

[37] J.M. Campbell, R.K. Ellis and C. Williams, Associated production of a Higgs boson at NNLO, JHEP 06 (2016) 179 [arXiv:1601.00658] [INSPIRE].

[38] L. Altenkamp, S. Dittmaier, R.V. Harlander, H. Rzehak and T.J.E. Zirke, Gluon-induced Higgs-strahlung at next-to-leading order QCD, JHEP 02 (2013) 078 [arXiv:1211.5015] [INSPIRE].

[39] B. Hespel, F. Maltoni and E. Vryonidou, Higgs and Z boson associated production via gluon fusion in the SM and the 2HDM, JHEP 06 (2015) 065 [arXiv:1503.01656] [INSPIRE].

[40] R.V. Harlander, A. Kulesza, V. Theeuwes and T. Zirke, Soft gluon resummation for gluon-induced Higgs Strahlung, JHEP 11 (2014) 082 [arXiv:1410.0217] [INSPIRE]. 
[41] R.V. Harlander, S. Liebler and T. Zirke, Higgs Strahlung at the Large Hadron Collider in the 2-Higgs-Doublet Model, JHEP 02 (2014) 023 [arXiv:1307.8122] [INSPIRE].

[42] O. Brein, R.V. Harlander and T.J.E. Zirke, vh@nnlo - Higgs Strahlung at hadron colliders, Comput. Phys. Commun. 184 (2013) 998 [arXiv:1210.5347] [InSPIRE].

[43] ATLAS collaboration, Measurements of b-jet tagging efficiency with the ATLAS detector using $t \bar{t}$ events at $\sqrt{s}=13 \mathrm{TeV}$, JHEP 08 (2018) 089 [arXiv: 1805.01845] [INSPIRE].

[44] ATLAS collaboration, Evaluation of theoretical uncertainties for simplified template cross section measurements of $V$-associated production of the Higgs boson, ATL-PHYS-PUB-2018-035 (2018).

[45] R. Contino, M. Ghezzi, C. Grojean, M. Mühlleitner and M. Spira, Effective Lagrangian for a light Higgs-like scalar, JHEP 07 (2013) 035 [arXiv: 1303.3876] [INSPIRE].

[46] G.F. Giudice, C. Grojean, A. Pomarol and R. Rattazzi, The Strongly-Interacting Light Higgs, JHEP 06 (2007) 045 [hep-ph/0703164] [INSPIRE].

[47] A. Alloul, B. Fuks and V. Sanz, Phenomenology of the Higgs effective Lagrangian via FeynRules, JHEP 04 (2014) 110 [arXiv:1310.5150] [INSPIRE].

[48] C. Hays, V. Sanz Gonzalez and G. Zemaityte, Constraining EFT parameters using simplified template cross sections, LHCHXSWG-INT-2017-001 (2017).

[49] J. Ellis, C.W. Murphy, V. Sanz and T. You, Updated Global SMEFT Fit to Higgs, Diboson and Electroweak Data, JHEP 06 (2018) 146 [arXiv: 1803.03252] [INSPIRE].

[50] ATLAS collaboration, ATLAS Computing Acknowledgements, ATL-GEN-PUB-2016-002 (2016). 


\section{The ATLAS collaboration}

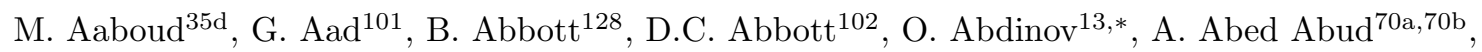
D.K. Abhayasinghe ${ }^{93}$, S.H. Abidi ${ }^{167}$, O.S. AbouZeid ${ }^{40}$, N.L. Abraham ${ }^{156}$, H. Abramowicz ${ }^{161}$, H. Abreu ${ }^{160}$, Y. Abulaiti ${ }^{6}$, B.S. Acharya ${ }^{66 a, 66 b, n}$, S. Adachi ${ }^{163}$, L. Adam ${ }^{99}$,

C. Adam Bourdarios ${ }^{132}$, L. Adamczyk ${ }^{83 a}$, L. Adamek $^{167}$, J. Adelman ${ }^{121}$, M. Adersberger ${ }^{114}$,

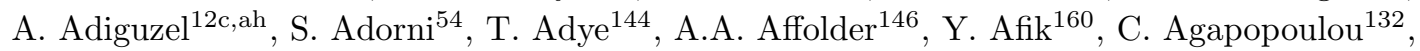
M.N. Agaras ${ }^{38}$, A. Aggarwal ${ }^{119}$, C. Agheorghiesei ${ }^{27 c}$, J.A. Aguilar-Saavedra 140f,140a,ag, F. Ahmadov ${ }^{79}$, G. Aielli73a,73b, S. Akatsuka ${ }^{85}$, T.P.A. Åkesson ${ }^{96}$, E. Akilli ${ }^{54}$, A.V. Akimov ${ }^{110}$, K. Al Khoury ${ }^{132}$, G.L. Alberghi ${ }^{23 b, 23 a}$, J. Albert ${ }^{176}$, M.J. Alconada Verzini ${ }^{88}$, S. Alderweireldt ${ }^{119}$, M. Aleksa ${ }^{36}$, I.N. Aleksandrov ${ }^{79}$, C. Alexa ${ }^{27 b}$, D. Alexandre ${ }^{19}$, T. Alexopoulos ${ }^{10}$, A. Alfonsi ${ }^{120}$, M. Alhroob ${ }^{128}$, B. Ali ${ }^{142}$, G. Alimonti ${ }^{68 a}$, J. Alison ${ }^{37}$, S.P. Alkire ${ }^{148}$, C. Allaire ${ }^{132}$, B.M.M. Allbrooke ${ }^{156}$, B.W. Allen ${ }^{131}$, P.P. Allport ${ }^{21}$, A. Aloisio ${ }^{69 a}$,69b, A. Alonso ${ }^{40}$, F. Alonso ${ }^{88}$, C. Alpigiani ${ }^{148}$, A.A. Alshehri ${ }^{57}$, M.I. Alstaty ${ }^{101}$, M. Alvarez Estevez ${ }^{98}$, B. Alvarez Gonzalez ${ }^{36}$,

D. Álvarez Piqueras ${ }^{174}$, M.G. Alviggi $69 \mathrm{a}, 69 \mathrm{~b}$, Y. Amaral Coutinho ${ }^{80 b}$, A. Ambler ${ }^{103}$, L. Ambroz ${ }^{135}$, C. Amelung ${ }^{26}$, D. Amidei ${ }^{105}$, S.P. Amor Dos Santos ${ }^{140 a, 140 c}$, S. Amoroso ${ }^{46}$, C.S. Amrouche ${ }^{54}$, F. $A^{78}$, C. Anastopoulos ${ }^{149}$, N. Andari ${ }^{145}$, T. Andeen ${ }^{11}$, C.F. Anders ${ }^{61 b}$, J.K. Anders ${ }^{20}$,

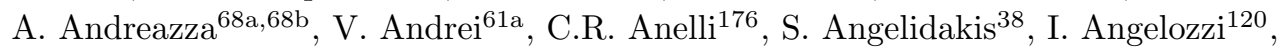
A. Angerami ${ }^{39}$, A.V. Anisenkov ${ }^{122 b, 122 a}$, A. Annovi ${ }^{71 a}$, C. Antel ${ }^{61 a}$, M.T. Anthony ${ }^{149}$, M. Antonelli ${ }^{51}$, D.J.A. Antrim ${ }^{171}$, F. Anulli ${ }^{72 a}$, M. Aoki ${ }^{81}$, J.A. Aparisi Pozo ${ }^{174}$,

L. Aperio Bella ${ }^{36}$, G. Arabidze ${ }^{106}$, J.P. Araque ${ }^{140 a}$, V. Araujo Ferraz ${ }^{80 b}$, R. Araujo Pereira ${ }^{80 b}$, A.T.H. Arce ${ }^{49}$, F.A. Arduh ${ }^{88}$, J-F. Arguin ${ }^{109}$, S. Argyropoulos ${ }^{77}$, J.-H. Arling 46 , A.J. Armbruster ${ }^{36}$, L.J. Armitage ${ }^{92}$, A. Armstrong ${ }^{171}$, O. Arnaez ${ }^{167}$, H. Arnold ${ }^{120}$, A. Artamonov ${ }^{111, *}$, G. Artoni ${ }^{135}$, S. Artz ${ }^{99}$, S. Asai ${ }^{163}$, N. Asbah ${ }^{59}$, E.M. Asimakopoulou ${ }^{172}$, L. Asquith ${ }^{156}$, K. Assamagan ${ }^{29}$, R. Astalos ${ }^{28 a}$, R.J. Atkin ${ }^{33 a}$, M. Atkinson ${ }^{173}$, N.B. Atlay ${ }^{151}$, H. Atmani ${ }^{132}$, K. Augsten ${ }^{142}$, G. Avolio ${ }^{36}$, R. Avramidou ${ }^{60 a}$, M.K. Ayoub ${ }^{15 a}$, A.M. Azoulay ${ }^{168 b}$, G. Azuelos ${ }^{109, a v}$, A.E. Baas ${ }^{61 a}$, M.J. Baca ${ }^{21}$, H. Bachacou ${ }^{145}$, K. Bachas ${ }^{67 a, 67 b}$, M. Backes ${ }^{135}$, F. Backman ${ }^{45 a, 45 b}$, P. Bagnaia ${ }^{72 a, 72 b}$, M. Bahmani ${ }^{84}$, H. Bahrasemani ${ }^{152}$, A.J. Bailey ${ }^{174}$, V.R. Bailey ${ }^{173}$, J.T. Baines ${ }^{144}$, M. Bajic ${ }^{40}$, C. Bakalis ${ }^{10}$, O.K. Baker ${ }^{183}$, P.J. Bakker ${ }^{120}$, D. Bakshi Gupta ${ }^{8}$, S. Balaji ${ }^{157}$, E.M. Baldin ${ }^{122 b, 122 a}$, P. Balek ${ }^{180}$, F. Balli ${ }^{145}$, W.K. Balunas ${ }^{135}$, J. Balz ${ }^{99}$, E. Banas ${ }^{84}$, A. Bandyopadhyay ${ }^{24}$, Sw. Banerjee ${ }^{181, i}$, A.A.E. Bannoura ${ }^{182}$, L. Barak ${ }^{161}$, W.M. Barbe ${ }^{38}$, E.L. Barberio ${ }^{104}$, D. Barberis ${ }^{55 b, 55 a}$, M. Barbero ${ }^{101}$, T. Barillari ${ }^{115}$,

M-S. Barisits ${ }^{36}$, J. Barkeloo ${ }^{131}$, T. Barklow ${ }^{153}$, R. Barnea ${ }^{160}$, S.L. Barnes ${ }^{60 c}$, B.M. Barnett ${ }^{144}$, R.M. Barnett ${ }^{18}$, Z. Barnovska-Blenessy ${ }^{60 a}$, A. Baroncelli ${ }^{60 a}$, G. Barone ${ }^{29}$, A.J. Barr ${ }^{135}$,

L. Barranco Navarro ${ }^{174}$, F. Barreiro ${ }^{98}$, J. Barreiro Guimarães da Costa ${ }^{15 a}$, R. Bartoldus ${ }^{153}$, G. Bartolini ${ }^{101}$, A.E. Barton ${ }^{89}$, P. Bartos ${ }^{28 a}$, A. Basalaev ${ }^{46}$, A. Bassalat ${ }^{132, \text { ap }, \text { R.L. Bates }}{ }^{57}$, S.J. Batista ${ }^{167}$, S. Batlamous ${ }^{35 e}$, J.R. Batley ${ }^{32}$, B. Batool ${ }^{151}$, M. Battaglia ${ }^{146}$, M. Bauce ${ }^{72 a, 72 b}$, F. Bauer ${ }^{145}$, K.T. Bauer ${ }^{171}$, H.S. Bawa ${ }^{31,1}$, J.B. Beacham ${ }^{49}$, T. Beau ${ }^{136}$, P.H. Beauchemin ${ }^{170}$, P. Bechtle ${ }^{24}$, H.C. Beck ${ }^{53}$, H.P. Beck ${ }^{20, \mathrm{q}}$, K. Becker ${ }^{52}$, M. Becker ${ }^{99}$, C. Becot ${ }^{46}$, A. Beddall ${ }^{12 \mathrm{~d}}$, A.J. Beddall ${ }^{12 a}$, V.A. Bednyakov ${ }^{79}$, M. Bedognetti ${ }^{120}$, C.P. Bee ${ }^{155}$, T.A. Beermann ${ }^{76}$,

M. Begalli ${ }^{80 b}$, M. Begel ${ }^{29}$, A. Behera ${ }^{155}$, J.K. Behr ${ }^{46}$, F. Beisiegel ${ }^{24}$, A.S. Bell ${ }^{94}$, G. Bella ${ }^{161}$, L. Bellagamba ${ }^{23 b}$, A. Bellerive ${ }^{34}$, P. Bellos ${ }^{9}$, K. Beloborodov ${ }^{122 b, 122 a}$, K. Belotskiy ${ }^{112}$, N.L. Belyaev ${ }^{112}$, O. Benary ${ }^{161, *}$, D. Benchekroun ${ }^{35 a}$, N. Benekos ${ }^{10}$, Y. Benhammou ${ }^{161}$, D.P. Benjamin 6 , M. Benoit ${ }^{54}$, J.R. Bensinger ${ }^{26}$, S. Bentvelsen ${ }^{120}$, L. Beresford ${ }^{135}$, M. Beretta ${ }^{51}$, D. Berge ${ }^{46}$, E. Bergeaas Kuutmann ${ }^{172}$, N. Berger ${ }^{5}$, B. Bergmann ${ }^{142}$, L.J. Bergsten ${ }^{26}$,

J. Beringer ${ }^{18}$, S. Berlendis ${ }^{7}$, N.R. Bernard ${ }^{102}$, G. Bernardi ${ }^{136}$, C. Bernius ${ }^{153}$, F.U. Bernlochner ${ }^{24}$, T. Berry ${ }^{93}$, P. Berta ${ }^{99}$, C. Bertella ${ }^{15 a}$, G. Bertoli ${ }^{45 a, 45 b}$, I.A. Bertram ${ }^{89}$, G.J. Besjes ${ }^{40}$, O. Bessidskaia Bylund ${ }^{182}$, N. Besson ${ }^{145}$, A. Bethani ${ }^{100}$, S. Bethke ${ }^{115}$, A. Betti ${ }^{24}$, A.J. Bevan ${ }^{92}$, 
J. Beyer ${ }^{115}$, R. Bi ${ }^{139}$, R.M. Bianchi ${ }^{139}$, O. Biebel ${ }^{114}$, D. Biedermann ${ }^{19}$, R. Bielski ${ }^{36}$, K. Bierwagen ${ }^{99}$, N.V. Biesuz ${ }^{71 a, 71 b}$, M. Biglietti ${ }^{74 a}$, T.R.V. Billoud ${ }^{109}$, M. Bindi $^{53}$, A. Bingul ${ }^{12 d}$, C. Bini ${ }^{72 a, 72 b}$, S. Biondi ${ }^{23 b, 23 a}$, M. Birman ${ }^{180}$, T. Bisanz ${ }^{53}$, J.P. Biswal ${ }^{161}$, A. Bitadze $^{100}$, C. Bittrich ${ }^{48}$, D.M. Bjergaard ${ }^{49}$, J.E. Black ${ }^{153}$, K.M. Black ${ }^{25}$, T. Blazek ${ }^{28 a}$, I. Bloch ${ }^{46}$, C. Blocker ${ }^{26}$, A. Blue ${ }^{57}$, U. Blumenschein ${ }^{92}$, G.J. Bobbink ${ }^{120}$, V.S. Bobrovnikov ${ }^{122 b, 122 a}$, S.S. Bocchetta ${ }^{96}$, A. Bocci ${ }^{49}$, D. Boerner ${ }^{46}$, D. Bogavac ${ }^{114}$, A.G. Bogdanchikov ${ }^{122 b, 122 a}$, C. Bohm ${ }^{45 a}$, V. Boisvert ${ }^{93}$, P. Bokan ${ }^{53,172}$, T. Bold ${ }^{83 a}$, A.S. Boldyrev ${ }^{113}$, A.E. Bolz ${ }^{61 b}$, M. Bomben ${ }^{136}$, M. Bona ${ }^{92}$, J.S. Bonilla ${ }^{131}$, M. Boonekamp ${ }^{145}$, H.M. Borecka-Bielska ${ }^{90}$, A. Borisov ${ }^{123}$, G. Borissov ${ }^{89}$, J. Bortfeldt ${ }^{36}$, D. Bortoletto ${ }^{135}$, V. Bortolotto ${ }^{73 a, 73 b}$, D. Boscherini2 ${ }^{23 b}$, M. Bosman ${ }^{14}$, J.D. Bossio Sola ${ }^{30}$, K. Bouaouda ${ }^{35 a}$, J. Boudreau ${ }^{139}$, E.V. Bouhova-Thacker ${ }^{89}$, D. Boumediene ${ }^{38}$, S.K. Boutle ${ }^{57}$, A. Boveia ${ }^{126}$, J. Boyd $^{36}$, D. Boye ${ }^{33 b}$, I.R. Boyko ${ }^{79}$, A.J. Bozson ${ }^{93}$, J. Bracinik ${ }^{21}$, N. Brahimi ${ }^{101}$, G. Brandt ${ }^{182}$, O. Brandt ${ }^{61 a}$, F. Braren ${ }^{46}$, U. Bratzler ${ }^{164}$, B. Brau ${ }^{102}$, J.E. Brau ${ }^{131}$, W.D. Breaden Madden ${ }^{57}$, K. Brendlinger ${ }^{46}$, L. Brenner ${ }^{46}$, R. Brenner ${ }^{172}$, S. Bressler ${ }^{180}$, B. Brickwedde ${ }^{99}$, D.L. Briglin ${ }^{21}$, D. Britton ${ }^{57}$, D. Britzger ${ }^{115}$, I. Brock ${ }^{24}$, R. Brock ${ }^{106}$, G. Brooijmans ${ }^{39}$, T. Brooks ${ }^{93}$, W.K. Brooks ${ }^{147 b}$, E. Brost ${ }^{121}$, J.H Broughton ${ }^{21}$, P.A. Bruckman de Renstrom ${ }^{84}$, D. Bruncko ${ }^{28 b}$, A. Bruni ${ }^{23 b}$, G. Bruni2 ${ }^{23 b}$, L.S. Bruni ${ }^{120}$, S. Bruno ${ }^{73 a, 73 b}$, B.H. Brunt ${ }^{32}$, M. Bruschi ${ }^{23 b}$, N. Bruscino ${ }^{139}$, P. Bryant ${ }^{37}$, L. Bryngemark ${ }^{96}$, T. Buanes ${ }^{17}$, Q. Buat ${ }^{36}$, P. Buchholz ${ }^{151}$, A.G. Buckley ${ }^{57}$, I.A. Budagov ${ }^{79}$, M.K. Bugge ${ }^{134}$, F. Bührer ${ }^{52}$, O. Bulekov ${ }^{112}$, T.J. Burch ${ }^{121}$, S. Burdin ${ }^{90}$, C.D. Burgard ${ }^{120}$, A.M. Burger ${ }^{129}$, B. Burghgrave ${ }^{8}$, K. Burka ${ }^{84}$, J.T.P. Burr ${ }^{46}$, V. Büscher ${ }^{99}$, E. Buschmann ${ }^{53}$, P.J. Bussey ${ }^{57}$, J.M. Butler ${ }^{25}$, C.M. Buttar ${ }^{57}$, J.M. Butterworth ${ }^{94}$, P. Butti ${ }^{36}$, W. Buttinger ${ }^{36}$, A. Buzatu ${ }^{158}$, A.R. Buzykaev ${ }^{122 b, 122 a}$, G. Cabras ${ }^{23 b, 23 a}$, S. Cabrera Urbán ${ }^{174}$, D. Caforio ${ }^{142}$, H. Cai ${ }^{173}$, V.M.M. Cairo ${ }^{153}$, O. Cakir ${ }^{4 a}$, N. Calace ${ }^{36}$, P. Calafiura ${ }^{18}$,

A. Calandri ${ }^{101}$, G. Calderini ${ }^{136}$, P. Calfayan ${ }^{65}$, G. Callea ${ }^{57}$, L.P. Caloba ${ }^{80 b}$, S. Calvente Lopez ${ }^{98}$, D. Calvet $^{38}$, S. Calvet ${ }^{38}$, T.P. Calvet ${ }^{155}$, M. Calvetti ${ }^{71 a, 71 b}$, R. Camacho Toro ${ }^{136}$, S. Camarda ${ }^{36}$, D. Camarero $\mathrm{Munoz}^{98}$, P. Camarri ${ }^{73 a, 73 b}$, D. Cameron ${ }^{134}$, R. Caminal Armadans ${ }^{102}$, C. Camincher ${ }^{36}$, S. Campana ${ }^{36}$, M. Campanelli ${ }^{94}$, A. Camplani ${ }^{40}$, A. Campoverde ${ }^{151}$, V. Canale ${ }^{69 a, 69 b}$, A. Canesse ${ }^{103}$, M. Cano Bret ${ }^{60 c}$, J. Cantero ${ }^{129}$, T. $\mathrm{Cao}^{161}$, Y. Cao $^{173}$, M.D.M. Capeans Garrido ${ }^{36}$, M. Capua ${ }^{41 b, 41 a}$, R. Cardarelli ${ }^{73 a}$, F.C. Cardillo ${ }^{149}$, I. Carli ${ }^{143}$, T. Carli $^{36}$, G. Carlino ${ }^{69 a}$, B.T. Carlson ${ }^{139}$, L. Carminati68a,68b, R.M.D. Carney ${ }^{45 a, 45 b}$, S. Caron ${ }^{119}$, E. Carquin ${ }^{147 b}$, S. Carrá ${ }^{68 a, 68 b}$, J.W.S. Carter ${ }^{167}$, M.P. Casado ${ }^{14, e}$, A.F. Casha ${ }^{167}$, D.W. Casper ${ }^{171}$, R. Castelijn ${ }^{120}$, F.L. Castillo ${ }^{174}$, V. Castillo Gimenez ${ }^{174}$, N.F. Castro ${ }^{140 a, 140 e}$, A. Catinaccio ${ }^{36}$, J.R. Catmore ${ }^{134}$, A. Cattai ${ }^{36}$, J. Caudron ${ }^{24}$, V. Cavaliere ${ }^{29}$, E. Cavallaro ${ }^{14}$, D. Cavalli ${ }^{68 a}$, M. Cavalli-Sforza ${ }^{14}$, V. Cavasinni ${ }^{71 a, 71 b}$, E. Celebi $^{12 b}$, F. Ceradini ${ }^{74 a, 74 b}$, L. Cerda Alberich ${ }^{174}$, A.S. Cerqueira ${ }^{80 a}$, A. Cerri ${ }^{156}$, L. Cerrito ${ }^{73 a}, 73 b$, F. Cerutti ${ }^{18}$, A. Cervelli23b,23a, S.A. Cetin ${ }^{12 b}$, A. Chafaq ${ }^{35 a}$, D. Chakraborty ${ }^{121}$, S.K. Chan ${ }^{59}$, W.S. Chan ${ }^{120}$, W.Y. Chan ${ }^{90}$, J.D. Chapman ${ }^{32}$, B. Chargeishvili1 ${ }^{159 b}$, D.G. Charlton ${ }^{21}$, C.C. Chau ${ }^{34}$, C.A. Chavez Barajas ${ }^{156}$, S. $\mathrm{Che}^{126}$, A. Chegwidden ${ }^{106}$, S. Chekanov ${ }^{6}$, S.V. Chekulaev ${ }^{168 a}$, G.A. Chelkov ${ }^{79, a u}$, M.A. Chelstowska ${ }^{36}$, B. Chen ${ }^{78}$, C. Chen ${ }^{60 a}$, C.H. Chen ${ }^{78}$, H. $\mathrm{Chen}^{29}$, J. Chen ${ }^{60 a}$, J. Chen ${ }^{39}$, S. Chen ${ }^{137}$, S.J. Chen ${ }^{15 c}$, X. Chen ${ }^{15 b, a t}$, Y. Chen ${ }^{82}$, Y-H. Chen ${ }^{46}$, H.C. Cheng ${ }^{63 a}$, H.J. Cheng ${ }^{15 a, 15 d}$, A. Cheplakov ${ }^{79}$, E. Cheremushkina ${ }^{123}$,

R. Cherkaoui El Moursli ${ }^{35 e}$, E. Cheu ${ }^{7}$, K. Cheung ${ }^{64}$, T.J.A. Chevalérias ${ }^{145}$, L. Chevalier $^{145}$, V. Chiarella ${ }^{51}$, G. Chiarelli ${ }^{71 a}$, G. Chiodini ${ }^{67 a}$, A.S. Chisholm ${ }^{36,21}$, A. Chitan ${ }^{27 b}$, I. Chiu ${ }^{163}$, Y.H. Chiu ${ }^{176}$, M.V. Chizhov ${ }^{79}$, K. Choi ${ }^{65}$, A.R. Chomont ${ }^{132}$, S. Chouridou ${ }^{162}$, Y.S. Chow ${ }^{120}$, M.C. $\mathrm{Chu}^{63 a}$, J. Chudoba ${ }^{141}$, A.J. Chuinard ${ }^{103}$, J.J. Chwastowski ${ }^{84}$, L. Chytka ${ }^{130}$, D. Cinca ${ }^{47}$, V. Cindro ${ }^{91}$, I.A. Cioară ${ }^{27 b}$, A. Ciocio ${ }^{18}$, F. Cirotto ${ }^{69 a, 69 b}$, Z.H. Citron ${ }^{180}$, M. Citterio ${ }^{68 a}$, B.M. Ciungu ${ }^{167}$, A. Clark ${ }^{54}$, M.R. Clark $^{39}$, P.J. Clark ${ }^{50}$, C. Clement ${ }^{45 a, 45 b}$, Y. Coadou ${ }^{101}$, M. Cobal ${ }^{66 a}, 66 c$, A. Coccaro ${ }^{55 b}$, J. Cochran ${ }^{78}$, H. Cohen ${ }^{161}$, A.E.C. Coimbra ${ }^{180}$, L. Colasurdo ${ }^{119}$, 
B. Cole ${ }^{39}$, A.P. Colijn ${ }^{120}$, J. Collot ${ }^{58}$, P. Conde Muiño ${ }^{140 a, f}$, E. Coniavitis ${ }^{52}$, S.H. Connell ${ }^{33 b}$, I.A. Connelly ${ }^{57}$, S. Constantinescu ${ }^{27 b}$, F. Conventi ${ }^{69 a}$,aw , A.M. Cooper-Sarkar ${ }^{135}$, F. Cormier ${ }^{175}$, K.J.R. Cormier ${ }^{167}$, L.D. Corpe ${ }^{94}$, M. Corradi ${ }^{72 a, 72 b}$, E.E. Corrigan ${ }^{96}$, F. Corriveau ${ }^{103, a c}$, A. Cortes-Gonzalez ${ }^{36}$, M.J. Costa ${ }^{174}$, F. Costanza $^{5}$, D. Costanzo $^{149}$, G. Cowan ${ }^{93}$, J.W. Cowley ${ }^{32}$, J. Crane ${ }^{100}$, K. Cranmer ${ }^{124}$, S.J. Crawley ${ }^{57}$, R.A. Creager ${ }^{137}$, S. Crépé-Renaudin ${ }^{58}$, F. Crescioli ${ }^{136}$, M. Cristinziani ${ }^{24}$, V. Croft ${ }^{120}$, G. Crosetti ${ }^{41 b, 41 a}$, A. Cueto $^{5}$, T. Cuhadar Donszelmann ${ }^{149}$, A.R. Cukierman ${ }^{153}$, S. Czekierda ${ }^{84}$, P. Czodrowski ${ }^{36}$, M.J. Da Cunha Sargedas De Sousa ${ }^{60 b}$, J.V. Da Fonseca Pinto ${ }^{80 b}$, C. Da Via ${ }^{100}$, W. Dabrowski ${ }^{83 a}$,

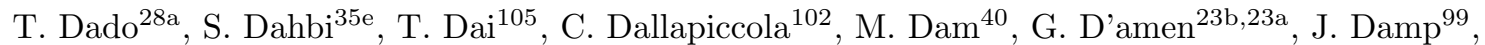
J.R. Dandoy ${ }^{137}$, M.F. Daneri ${ }^{30}$, N.P. Dang ${ }^{181}$, N.D Dann ${ }^{100}$, M. Danninger ${ }^{175}$, V. Dao ${ }^{36}$, G. Darbo ${ }^{55 b}$, O. Dartsi ${ }^{5}$, A. Dattagupta ${ }^{131}$, T. Daubney ${ }^{46}$, S. D'Auria ${ }^{68 a, 68 b}$, W. Davey ${ }^{24}$, C. David ${ }^{46}$, T. Davidek ${ }^{143}$, D.R. Davis ${ }^{49}$, E. Dawe ${ }^{104}$, I. Dawson ${ }^{149}$, K. De ${ }^{8}$, R. De Asmundis ${ }^{69 a}$,

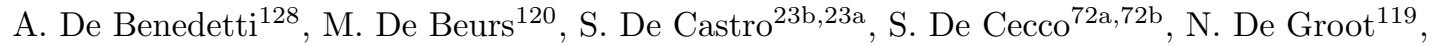
P. de Jong ${ }^{120}$, H. De la Torre ${ }^{106}$, A. De Maria ${ }^{15 c}$, D. De Pedis ${ }^{72 a}$, A. De Salvo ${ }^{72 a}$, U. De Sanctis ${ }^{73 a}, 73 b$, M. De Santis ${ }^{73 a}, 73 b$, A. De Santo ${ }^{156}$, K. De Vasconcelos Corga ${ }^{101}$, J.B. De Vivie De Regie ${ }^{132}$, C. Debenedetti ${ }^{146}$, D.V. Dedovich ${ }^{79}$, A.M. Deiana ${ }^{42}$, M. Del Gaudio ${ }^{41 b, 41 a}$, J. Del Peso ${ }^{98}$, Y. Delabat Diaz ${ }^{46}$, D. Delgove ${ }^{132}$, F. Deliot ${ }^{145}$, C.M. Delitzsch ${ }^{7}$, M. Della Pietra ${ }^{69 a, 69 b}$, D. Della Volpe ${ }^{54}$, A. Dell'Acqua ${ }^{36}$, L. Dell'Asta ${ }^{25}$, M. Delmastro ${ }^{5}$, C. Delporte ${ }^{132}$, P.A. Delsart ${ }^{58}$, D.A. DeMarco ${ }^{167}$, S. Demers ${ }^{183}$, M. Demichev ${ }^{79}$, G. Demontigny ${ }^{109}$, S.P. Denisov ${ }^{123}$, D. Denysiuk ${ }^{120}$, L. D'Eramo ${ }^{136}$, D. Derendarz ${ }^{84}$,

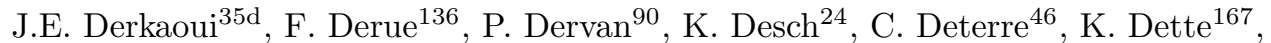
M.R. Devesa ${ }^{30}$, P.O. Deviveiros ${ }^{36}$, A. Dewhurst ${ }^{144}$, S. Dhaliwal ${ }^{26}$, F.A. Di Bello ${ }^{54}$, A. Di Ciaccio ${ }^{73 a}, 73 b$, L. Di Ciaccio 5 , W.K. Di Clemente ${ }^{137}$, C. Di Donato69a,69b , A. Di Girolamo ${ }^{36}$, G. Di Gregorio $71 \mathrm{a}, 71 b$, B. Di Micco ${ }^{74 a, 74 b}$, R. Di Nardo ${ }^{102}$, K.F. Di Petrillo ${ }^{59}$, R. Di Sipio ${ }^{167}$, D. Di Valentino ${ }^{34}$, C. Diaconu ${ }^{101}$, F.A. $\operatorname{Dias}^{40}$, T. Dias Do Vale ${ }^{140 a, 140 e}$, M.A. Diaz ${ }^{147 a}$, J. Dickinson ${ }^{18}$, E.B. Diehl ${ }^{105}$, J. Dietrich ${ }^{19}$, S. Díez Cornell ${ }^{46}$, A. Dimitrievska ${ }^{18}$, W. Ding ${ }^{15 b}$, J. Dingfelder ${ }^{24}$, F. Dittus ${ }^{36}$, F. Djama ${ }^{101}$, T. Djobava ${ }^{159 b}$, J.I. Djuvsland ${ }^{17}$, M.A.B. Do Vale ${ }^{80 c}$, M. Dobre ${ }^{27 b}$, D. Dodsworth ${ }^{26}$, C. Doglioni ${ }^{96}$, J. Dolejsi ${ }^{143}$, Z. Dolezal ${ }^{143}$, M. Donadelli ${ }^{80 d}$, J. Donini ${ }^{38}$, A. D'onofrio ${ }^{92}$, M. D’Onofrio ${ }^{90}$, J. Dopke ${ }^{144}$, A. Doria ${ }^{69 a}$, M.T. Dova ${ }^{88}$,

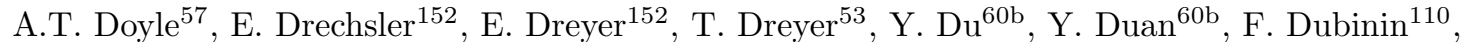
M. Dubovsky ${ }^{28 a}$, A. Dubreuil ${ }^{54}$, E. Duchovni ${ }^{180}$, G. Duckeck ${ }^{114}$, A. Ducourthial ${ }^{136}$, O.A. Ducu ${ }^{109, w}$, D. Duda ${ }^{115}$, A. Dudarev ${ }^{36}$, A.C. Dudder ${ }^{99}$, E.M. Duffield ${ }^{18}$, L. Duflot ${ }^{132}$, M. Dührssen ${ }^{36}$, C. Dülsen ${ }^{182}$, M. Dumancic ${ }^{180}$, A.E. Dumitriu ${ }^{27 b}$, A.K. Duncan ${ }^{57}$, M. Dunford ${ }^{61 a}$, A. Duperrin ${ }^{101}$, H. Duran Yildiz ${ }^{4 a}$, M. Düren ${ }^{56}$, A. Durglishvili ${ }^{159 b}$, D. Duschinger ${ }^{48}$, B. Dutta ${ }^{46}$, D. Duvnjak ${ }^{1}$, G.I. Dyckes ${ }^{137}$, M. Dyndal ${ }^{46}$, S. Dysch ${ }^{100}$, B.S. Dziedzic ${ }^{84}$, K.M. Ecker ${ }^{115}$, R.C. Edgar ${ }^{105}$, T. Eifert ${ }^{36}$, G. Eigen ${ }^{17}$, K. Einsweiler ${ }^{18}$, T. Ekelof ${ }^{172}$, M. El Kacimi ${ }^{35 c}$, R. El Kosseifi ${ }^{101}$, V. Ellajosyula ${ }^{172}$, M. Ellert ${ }^{172}$, F. Ellinghaus ${ }^{182}$, A.A. Elliot ${ }^{92}$, N. Ellis ${ }^{36}$, J. Elmsheuser ${ }^{29}$, M. Elsing ${ }^{36}$, D. Emeliyanov ${ }^{144}$, A. Emerman ${ }^{39}$, Y. Enari ${ }^{163}$, J.S. Ennis ${ }^{178}$, M.B. Epland ${ }^{49}$, J. Erdmann ${ }^{47}$, A. Ereditato ${ }^{20}$, M. Escalier ${ }^{132}$, C. Escobar ${ }^{174}$,

O. Estrada Pastor ${ }^{174}$, A.I. Etienvre ${ }^{145}$, E. Etzion ${ }^{161}$, H. Evans ${ }^{65}$, A. Ezhilov ${ }^{138}$, M. Ezzi ${ }^{35 e}$, F. Fabbri ${ }^{57}$, L. Fabbri ${ }^{23 b, 23 a}$, V. Fabiani ${ }^{119}$, G. Facini ${ }^{94}$, R.M. Faisca Rodrigues Pereira ${ }^{140 a}$, R.M. Fakhrutdinov ${ }^{123}$, S. Falciano ${ }^{72 a}$, P.J. Falke ${ }^{5}$, S. Falke ${ }^{5}$, J. Faltova ${ }^{143}$, Y. Fang ${ }^{15 a}$, Y. Fang ${ }^{15 a}$, G. Fanourakis ${ }^{44}$, M. Fanti ${ }^{68 a, 68 b}$, A. Farbin ${ }^{8}$, A. Farilla ${ }^{74 a}$, E.M. Farina ${ }^{70 a, 70 b}$, T. Farooque ${ }^{106}$, S. Farrell ${ }^{18}$, S.M. Farrington ${ }^{178}$, P. Farthouat ${ }^{36}$, F. Fassi ${ }^{35 e}$, P. Fassnacht ${ }^{36}$, D. Fassouliotis ${ }^{9}$, M. Faucci Giannelli ${ }^{50}$, W.J. Fawcett ${ }^{32}$, L. Fayard ${ }^{132}$, O.L. Fedin ${ }^{138, o}$, W. Fedorko ${ }^{175}$, M. Feickert ${ }^{42}$, S. Feigl ${ }^{134}$, L. Feligioni ${ }^{101}$, A. Fell ${ }^{149}$, C. Feng ${ }^{60 b}$, E.J. Feng ${ }^{36}$, M. Feng ${ }^{49}$, M.J. Fenton ${ }^{57}$, A.B. Fenyuk ${ }^{123}$, J. Ferrando ${ }^{46}$, A. Ferrari ${ }^{172}$, P. Ferrari ${ }^{120}$, R. Ferrari ${ }^{70 a}$, D.E. Ferreira de Lima ${ }^{61 b}$, A. Ferrer ${ }^{174}$, D. Ferrere ${ }^{54}$, C. Ferretti ${ }^{105}$, F. Fiedler ${ }^{99}$, 
A. Filipčič ${ }^{91}$, F. Filthaut ${ }^{119}$, K.D. Finelli ${ }^{25}$, M.C.N. Fiolhais ${ }^{140 a}$, L. Fiorini ${ }^{174}$, C. Fischer ${ }^{14}$, F. Fischer ${ }^{114}$, W.C. Fisher ${ }^{106}$, I. Fleck ${ }^{151}$, P. Fleischmann ${ }^{105}$, R.R.M. Fletcher ${ }^{137}$, T. Flick ${ }^{182}$, B.M. Flierl ${ }^{114}$, L.F. Flores ${ }^{137}$, L.R. Flores Castillo ${ }^{63 a}$, F.M. Follega ${ }^{75 a, 75 b}$, N. Fomin ${ }^{17}$, G.T. Forcolin ${ }^{75 a, 75 b}$, A. Formica ${ }^{145}$, F.A. Förster ${ }^{14}$, A.C. Forti ${ }^{100}$, A.G. Foster ${ }^{21}$, D. Fournier ${ }^{132}$, H. Fox ${ }^{89}$, S. Fracchia ${ }^{149}$, P. Francavilla ${ }^{71 a, 71 b}$, M. Franchini ${ }^{23 b, 23 a}$, S. Franchino ${ }^{61 a}$, D. Francis ${ }^{36}$, L. Franconi ${ }^{20}$, M. Franklin ${ }^{59}$, M. Frate ${ }^{171}$, A.N. Fray ${ }^{92}$, B. Freund ${ }^{109}$, W.S. Freund ${ }^{80 b}$, E.M. Freundlich ${ }^{47}$, D.C. Frizzell ${ }^{128}$, D. Froidevaux ${ }^{36}$, J.A. Frost ${ }^{135}$, C. Fukunaga ${ }^{164}$, E. Fullana Torregrosa ${ }^{174}$, E. Fumagalli ${ }^{55 b, 55 a}$, T. Fusayasu ${ }^{116}$, J. Fuster ${ }^{174}$, A. Gabrielli ${ }^{23 b, 23 a,}$ A. Gabrielli ${ }^{18}$, G.P. Gach ${ }^{83 a}$, S. Gadatsch ${ }^{54}$, P. Gadow ${ }^{115}$, G. Gagliardi ${ }^{55 b, 55 a}$, L.G. Gagnon ${ }^{109}$, C. Galea ${ }^{27 b}$, B. Galhardo ${ }^{140 a, 140 c}$, E.J. Gallas ${ }^{135}$, B.J. Gallop ${ }^{144}$, P. Gallus ${ }^{142}$, G. Galster ${ }^{40}$, R. Gamboa Goni ${ }^{92}$, K.K. Gan ${ }^{126}$, S. Ganguly ${ }^{180}$, J. Gao ${ }^{60 a}$, Y. Gao ${ }^{90}$, Y.S. Gao ${ }^{31,1}$, C. García ${ }^{174}$, J.E. García Navarro ${ }^{174}$, J.A. García Pascual ${ }^{15 a}$, C. Garcia-Argos ${ }^{52}$, M. Garcia-Sciveres ${ }^{18}$, R.W. Gardner ${ }^{37}$, N. Garelli ${ }^{153}$, S. Gargiulo ${ }^{52}$, V. Garonne ${ }^{134}$, A. Gaudiello55b,55a, G. Gaudio ${ }^{70 a}$, I.L. Gavrilenko ${ }^{110}$, A. Gavrilyuk ${ }^{111}$, C. Gay ${ }^{175}$, G. Gaycken ${ }^{24}$, E.N. Gazis ${ }^{10}$, C.N.P. Gee ${ }^{144}$, J. Geisen ${ }^{53}$, M. Geisen ${ }^{99}$, M.P. Geisler ${ }^{61 a}$, C. Gemme ${ }^{55 b}$, M.H. Genest ${ }^{58}$, C. Geng ${ }^{105}$, S. Gentile ${ }^{72 a}, 72 b$, S. George ${ }^{93}$, T. Geralis ${ }^{44}$, D. Gerbaudo ${ }^{14}$, L.O. Gerlach ${ }^{53}$, G. Gessner ${ }^{47}$, S. Ghasemi ${ }^{151}$, M. Ghasemi Bostanabad ${ }^{176}$, M. Ghneimat ${ }^{24}$, A. Ghosh ${ }^{77}$, B. Giacobbe ${ }^{23 b}$, S. Giagu ${ }^{72 a, 72 b}$, N. Giangiacomi ${ }^{23 b, 23 a}$, P. Giannetti ${ }^{71 a}$, A. Giannini69a,69b, S.M. Gibson ${ }^{93}$, M. Gignac ${ }^{146}$, D. Gillberg ${ }^{34}$, G. Gilles ${ }^{182}$, D.M. Gingrich ${ }^{3, a v}$, M.P. Giordani ${ }^{66 a, 66 c}$, F.M. Giorgi ${ }^{23 b}$, P.F. Giraud ${ }^{145}$, G. Giugliarelli66a,66c , D. Giugni ${ }^{68 a}$, F. Giuli ${ }^{135}$, M. Giulini ${ }^{61 b}$, S. Gkaitatzis ${ }^{162}$, I. Gkialas ${ }^{9, h}$, E.L. Gkougkousis ${ }^{14}$, P. Gkountoumis ${ }^{10}$, L.K. Gladilin ${ }^{113}$, C. Glasman ${ }^{98}$, J. Glatzer ${ }^{14}$, P.C.F. Glaysher ${ }^{46}$, A. Glazov ${ }^{46}$, M. Goblirsch-Kolb ${ }^{26}$, S. Goldfarb ${ }^{104}$, T. Golling ${ }^{54}$, D. Golubkov ${ }^{123}$, A. Gomes ${ }^{140 a, 140 b}$, R. Goncalves Gama ${ }^{53}$, R. Gonçalo ${ }^{140 a, 140 b}$, G. Gonella ${ }^{52}$, L. Gonella ${ }^{21}$, A. Gongadze ${ }^{79}$, F. Gonnella ${ }^{21}$, J.L. Gonski ${ }^{59}$,

S. González de la $\mathrm{Hoz}^{174}$, S. Gonzalez-Sevilla ${ }^{54}$, G.R. Gonzalvo Rodriguez ${ }^{174}$, L. Goossens ${ }^{36}$, P.A. Gorbounov ${ }^{111}$, H.A. Gordon ${ }^{29}$, B. Gorini ${ }^{36}$, E. Gorini ${ }^{67 a}{ }^{67 b}$, A. Gorišek ${ }^{91}$, A.T. Goshaw ${ }^{49}$, C. Gössling ${ }^{47}$, M.I. Gostkin ${ }^{79}$, C.A. Gottardo ${ }^{24}$, C.R. Goudet ${ }^{132}$, D. Goujdami ${ }^{35 c}$, A.G. Goussiou ${ }^{148}$, N. Govender ${ }^{33 b, a}$, C. Goy ${ }^{5}$, E. Gozani ${ }^{160}$, I. Grabowska-Bold ${ }^{83 a}$, P.O.J. Gradin ${ }^{172}$, E.C. Graham ${ }^{90}$, J. Gramling ${ }^{171}$, E. Gramstad ${ }^{134}$, S. Grancagnolo ${ }^{19}$, M. Grandi ${ }^{156}$, V. Gratchev ${ }^{138}$, P.M. Gravila ${ }^{27 f}$, F.G. Gravili ${ }^{67 a, 67 b}$, C. Gray ${ }^{57}$, H.M. Gray ${ }^{18}$, C. Grefe ${ }^{24}$, K. Gregersen ${ }^{96}$, I.M. Gregor ${ }^{46}$, P. Grenier ${ }^{153}$, K. Grevtsov ${ }^{46}$, N.A. Grieser ${ }^{128}$, J. Griffiths ${ }^{8}$, A.A. Grillo ${ }^{146}$, K. Grimm ${ }^{31, k}$, S. Grinstein ${ }^{14, x}$, J.-F. Grivaz ${ }^{132}$, S. Groh ${ }^{99}$, E. Gross ${ }^{180}$, J. Grosse-Knetter ${ }^{53}$, Z.J. Grout ${ }^{94}$, C. Grud ${ }^{105}$, A. Grummer ${ }^{118}$, L. Guan ${ }^{105}$, W. Guan ${ }^{181}$, J. Guenther ${ }^{36}$, A. Guerguichon ${ }^{132}$, F. Guescini ${ }^{168 a}$, D. Guest ${ }^{171}$, R. Gugel ${ }^{52}$,

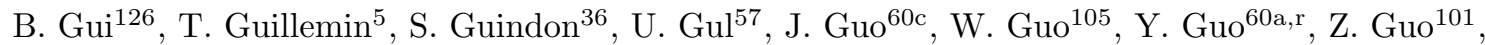

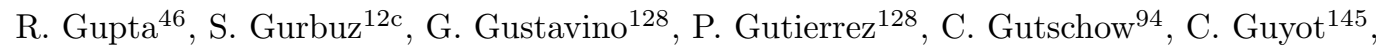
M.P. Guzik ${ }^{83 a}$, C. Gwenlan ${ }^{135}$, C.B. Gwilliam ${ }^{90}$, A. Haas ${ }^{124}$, C. Haber ${ }^{18}$, H.K. Hadavand ${ }^{8}$, N. Haddad ${ }^{35 e}$, A. Hadef ${ }^{60 a}$, S. Hageböck ${ }^{36}$, M. Hagihara ${ }^{169}$, M. Haleem ${ }^{177}$, J. Haley ${ }^{129}$, G. Halladjian ${ }^{106}$, G.D. Hallewell ${ }^{101}$, K. Hamacher ${ }^{182}$, P. Hamal ${ }^{130}$, K. Hamano ${ }^{176}$,

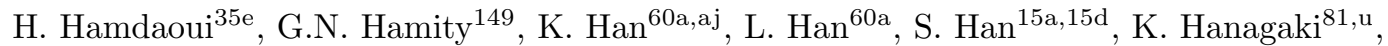
M. Hance ${ }^{146}$, D.M. Handl ${ }^{114}$, B. Haney ${ }^{137}$, R. Hankache ${ }^{136}$, P. Hanke ${ }^{61 a}$, E. Hansen ${ }^{96}$, J.B. Hansen ${ }^{40}$, J.D. Hansen ${ }^{40}$, M.C. Hansen ${ }^{24}$, P.H. Hansen ${ }^{40}$, E.C. Hanson ${ }^{100}$, K. Hara ${ }^{169}$, A.S. Hard ${ }^{181}$, T. Harenberg ${ }^{182}$, S. Harkusha ${ }^{107}$, P.F. Harrison ${ }^{178}$, N.M. Hartmann ${ }^{114}$, Y. Hasegawa ${ }^{150}$, A. Hasib ${ }^{50}$, S. Hassani ${ }^{145}$, S. Haug ${ }^{20}$, R. Hauser ${ }^{106}$, L. Hauswald ${ }^{48}$, L.B. Havener ${ }^{39}$, M. Havranek ${ }^{142}$, C.M. Hawkes ${ }^{21}$, R.J. Hawkings ${ }^{36}$, D. Hayden ${ }^{106}$, C. Hayes ${ }^{155}$, R.L. Hayes ${ }^{175}$, C.P. Hays ${ }^{135}$, J.M. Hays ${ }^{92}$, H.S. Hayward ${ }^{90}$, S.J. Haywood ${ }^{144}$, F. He ${ }^{60 a}$,

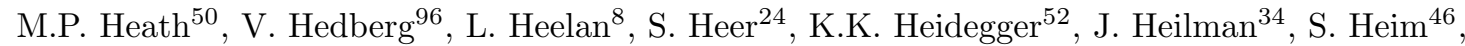
T. Heim ${ }^{18}$, B. Heinemann ${ }^{46, a q}$, J.J. Heinrich ${ }^{131}$, L. Heinrich ${ }^{36}$, C. Heinz ${ }^{56}$, J. Hejbal ${ }^{141}$, 
L. Helary ${ }^{61 b}$, A. Held ${ }^{175}$, S. Hellesund ${ }^{134}$, C.M. Helling ${ }^{146}$, S. Hellman ${ }^{45 a, 45 b}$, C. Helsens ${ }^{36}$, R.C.W. Henderson ${ }^{89}$, Y. Heng ${ }^{181}$, S. Henkelmann ${ }^{175}$, A.M. Henriques Correia ${ }^{36}$, G.H. Herbert ${ }^{19}$, H. Herde ${ }^{26}$, V. Herget ${ }^{177}$, Y. Hernández Jiménez ${ }^{33 c}$, H. Herr ${ }^{99}$, M.G. Herrmann ${ }^{114}$,

T. Herrmann ${ }^{48}$, G. Herten ${ }^{52}$, R. Hertenberger ${ }^{114}$, L. Hervas ${ }^{36}$, T.C. Herwig ${ }^{137}$, G.G. Hesketh ${ }^{94}$, N.P. Hessey ${ }^{168 a}$, A. Higashida ${ }^{163}$, S. Higashino ${ }^{81}$, E. Higón-Rodriguez ${ }^{174}$, K. Hildebrand ${ }^{37}$, E. Hill ${ }^{176}$, J.C. Hill ${ }^{32}$, K.K. Hill ${ }^{29}$, K.H. Hiller ${ }^{46}$, S.J. Hillier ${ }^{21}$, M. Hils ${ }^{48}$, I. Hinchliffe ${ }^{18}$, F. Hinterkeuser ${ }^{24}$, M. Hirose ${ }^{133}$, S. Hirose ${ }^{52}$, D. Hirschbuehl ${ }^{182}$, B. Hiti ${ }^{91}$, O. Hladik ${ }^{141}$, D.R. Hlaluku ${ }^{33 c}$, X. Hoad ${ }^{50}$, J. Hobbs ${ }^{155}$, N. Hod ${ }^{180}$, M.C. Hodgkinson ${ }^{149}$, A. Hoecker ${ }^{36}$, F. Hoenig ${ }^{114}$, D. Hohn ${ }^{52}$, D. Hohov ${ }^{132}$, T.R. Holmes ${ }^{37}$, M. Holzbock ${ }^{114}$, L.B.A.H Hommels ${ }^{32}$, S. Honda ${ }^{169}$, T. Honda ${ }^{81}$, T.M. Hong ${ }^{139}$, A. Hönle ${ }^{115}$, B.H. Hooberman ${ }^{173}$, W.H. Hopkins ${ }^{6}$, Y. Horii ${ }^{117}$, P. Horn ${ }^{48}$, A.J. Horton ${ }^{152}$, L.A. Horyn ${ }^{37}$, J-Y. Hostachy ${ }^{58}$, A. Hostiuc ${ }^{148}$, S. Hou ${ }^{158}$,

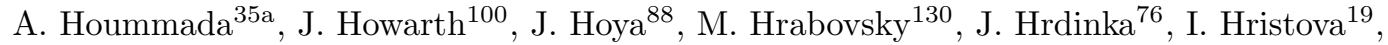
J. Hrivnac ${ }^{132}$, A. Hrynevich ${ }^{108}$, T. Hryn'ova ${ }^{5}$, P.J. Hsu ${ }^{64}$, S.-C. Hsu ${ }^{148}$, Q. Hu ${ }^{29}$, S. Hu ${ }^{60 c}$, Y. Huang ${ }^{15 a}$, Z. Hubacek ${ }^{142}$, F. Hubaut ${ }^{101}$, M. Huebner ${ }^{24}$, F. Huegging ${ }^{24}$, T.B. Huffman ${ }^{135}$, M. Huhtinen ${ }^{36}$, R.F.H. Hunter ${ }^{34}$, P. Huo ${ }^{155}$, A.M. Hupe ${ }^{34}$, N. Huseynov ${ }^{79, \text { ae }}$, J. Huston ${ }^{106}$, J. Huth ${ }^{59}$, R. Hyneman ${ }^{105}$, S. Hyrych ${ }^{28 a}$, G. Iacobucci ${ }^{54}$, G. Iakovidis ${ }^{29}$, I. Ibragimov ${ }^{151}$, L. Iconomidou-Fayard ${ }^{132}$, Z. Idrissi ${ }^{35 e}$, P.I. Iengo ${ }^{36}$, R. Ignazzi ${ }^{40}$, O. Igonkina ${ }^{120, z}$, R. Iguchi ${ }^{163}$, T. Iizawa ${ }^{54}$, Y. Ikegami ${ }^{81}$, M. Ikeno ${ }^{81}$, D. Iliadis ${ }^{162}$, N. Ilic ${ }^{119}$, F. Iltzsche ${ }^{48}$, G. Introzzi ${ }^{70 a, 70 b}$,

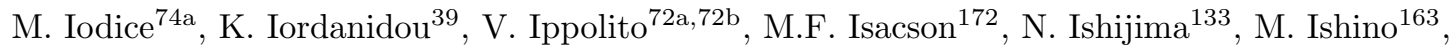
M. Ishitsuka ${ }^{165}$, W. Islam ${ }^{129}$, C. Issever ${ }^{135}$, S. Istin ${ }^{160}$, F. Ito ${ }^{169}$, J.M. Iturbe Ponce ${ }^{63 a}$,

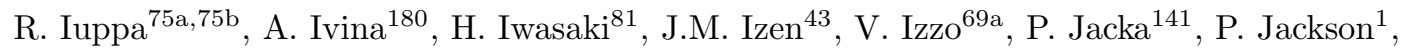
R.M. Jacobs ${ }^{24}$, V. Jain ${ }^{2}$, G. Jäkel ${ }^{182}$, K.B. Jakobi ${ }^{99}$, K. Jakobs ${ }^{52}$, S. Jakobsen ${ }^{76}$, T. Jakoubek ${ }^{141}$, J. Jamieson ${ }^{57}$, D.O. Jamin ${ }^{129}$, R. Jansky ${ }^{54}$, J. Janssen ${ }^{24}$, M. Janus ${ }^{53}$, P.A. Janus ${ }^{83 a}$, G. Jarlskog ${ }^{96}$, N. Javadov ${ }^{79, \text { ae }}$, T. Javůrek ${ }^{36}$, M. Javurkova ${ }^{52}$, F. Jeanneau ${ }^{145}$, L. Jeanty ${ }^{131}$, J. Jejelava ${ }^{159 a, \text { af }}$, A. Jelinskas ${ }^{178}$, P. Jenni ${ }^{52, b}$, J. Jeong ${ }^{46}$, N. Jeong ${ }^{46}$, S. Jézéquel ${ }^{5}$, H. Ji ${ }^{181}$, J. Jia ${ }^{155}$, H. Jiang ${ }^{78}$, Y. Jiang ${ }^{60 a}$, Z. Jiang ${ }^{153, p}$, S. Jiggins ${ }^{52}$, F.A. Jimenez Morales ${ }^{38}$, J. Jimenez Pena ${ }^{174}$, S. Jin ${ }^{15 c}$, A. Jinaru ${ }^{27 b}$, O. Jinnouchi ${ }^{165}$, H. Jivan ${ }^{33 c}$, P. Johansson ${ }^{149}$, K.A. Johns ${ }^{7}$, C.A. Johnson ${ }^{65}$, K. Jon-And ${ }^{45 a, 45 b}$, R.W.L. Jones ${ }^{89}$, S.D. Jones ${ }^{156}$, S. Jones ${ }^{7}$, T.J. Jones ${ }^{90}$, J. Jongmanns ${ }^{61 a}$, P.M. Jorge ${ }^{140 a, 140 b}$, J. Jovicevic ${ }^{168 a}$, X. Ju ${ }^{18}$, J.J. Junggeburth ${ }^{115}$, A. Juste Rozas ${ }^{14, x}$, A. Kaczmarska ${ }^{84}$, M. Kado ${ }^{132}$, H. Kagan ${ }^{126}$, M. Kagan ${ }^{153}$, T. Kaji179, E. Kajomovitz ${ }^{160}$, C.W. Kalderon ${ }^{96}$, A. Kaluza ${ }^{99}$, A. Kamenshchikov ${ }^{123}$, L. Kanjir ${ }^{91}$, Y. Kano ${ }^{163}$, V.A. Kantserov ${ }^{112}$, J. Kanzaki ${ }^{81}$, L.S. Kaplan ${ }^{181}$, D. Kar ${ }^{33 c}$, M.J. Kareem ${ }^{168 b}$, E. Karentzos ${ }^{10}$, S.N. Karpov ${ }^{79}$, Z.M. Karpova ${ }^{79}$, V. Kartvelishvili ${ }^{89}$, A.N. Karyukhin ${ }^{123}$, L. Kashif ${ }^{181}$, R.D. Kass ${ }^{126}$, A. Kastanas ${ }^{45 a, 45 b}$, Y. Kataoka ${ }^{163}$, C. Kato ${ }^{60 d, 60 c}$, J. Katzy ${ }^{46}$, K. Kawade ${ }^{82}$, K. Kawagoe ${ }^{87}$, T. Kawaguchi ${ }^{117}$, T. Kawamoto ${ }^{163}$, G. Kawamura ${ }^{53}$, E.F. Kay ${ }^{176}$, V.F. Kazanin ${ }^{122 b, 122 a}$, R. Keeler ${ }^{176}$, R. Kehoe ${ }^{42}$, J.S. Keller ${ }^{34}$, E. Kellermann ${ }^{96}$, J.J. Kempster ${ }^{21}$, J. Kendrick ${ }^{21}$, O. Kepka ${ }^{141}$, S. Kersten ${ }^{182}$, B.P. Kerševan ${ }^{91}$, S. Ketabchi Haghighat ${ }^{167}$, R.A. Keyes ${ }^{103}$, M. Khader ${ }^{173}$, F. Khalil-Zada ${ }^{13}$, A. Khanov ${ }^{129}$, A.G. Kharlamov ${ }^{122 b, 122 a}$, T. Kharlamova ${ }^{122 b, 122 a}$, E.E. Khoda ${ }^{175}$, A. Khodinov ${ }^{166}$, T.J. Khoo ${ }^{54}$, E. Khramov ${ }^{79}$, J. Khubua ${ }^{159 b}$, S. Kido ${ }^{82}$, M. Kiehn ${ }^{54}$, C.R. Kilby ${ }^{93}$, Y.K. Kim ${ }^{37}$, N. Kimura ${ }^{66 a, 66 c}$, O.M. Kind ${ }^{19}$, B.T. King ${ }^{90, *}$, D. Kirchmeier ${ }^{48}$, J. Kirk ${ }^{144}$, A.E. Kiryunin ${ }^{115}$, T. Kishimoto ${ }^{163}$, V. Kitali ${ }^{46}$, O. Kivernyk ${ }^{5}$, E. Kladiva ${ }^{28 b, *}$, T. Klapdor-Kleingrothaus ${ }^{52}$, M.H. Klein ${ }^{105}$, M. Klein ${ }^{90}$, U. Klein ${ }^{90}$, K. Kleinknecht ${ }^{99}$, P. Klimek ${ }^{121}$, A. Klimentov ${ }^{29}$, T. Klingl ${ }^{24}$, T. Klioutchnikova ${ }^{36}$, F.F. Klitzner ${ }^{114}$, P. Kluit ${ }^{120}$, S. Kluth ${ }^{115}$, E. Kneringer ${ }^{76}$, E.B.F.G. Knoops ${ }^{101}$, A. Knue ${ }^{52}$, D. Kobayashi ${ }^{87}$, T. Kobayashi ${ }^{163}$, M. Kobel ${ }^{48}$, M. Kocian ${ }^{153}$, P. Kodys ${ }^{143}$, P.T. Koenig ${ }^{24}$, T. Koffas ${ }^{34}$, N.M. Köhler ${ }^{115}$, T. Koi ${ }^{153}$, M. Kolb ${ }^{61 b}$, I. Koletsou ${ }^{5}$, T. Kondo ${ }^{81}$,

N. Kondrashova ${ }^{60 c}$, K. Köneke ${ }^{52}$, A.C. König ${ }^{119}$, T. Kono ${ }^{125}$, R. Konoplich ${ }^{124, a m}$, V. Konstantinides ${ }^{94}$, N. Konstantinidis ${ }^{94}$, B. Konya ${ }^{96}$, R. Kopeliansky ${ }^{65}$, S. Koperny ${ }^{83 a}$, 
K. Korcyl ${ }^{84}$, K. Kordas ${ }^{162}$, G. Koren ${ }^{161}$, A. Korn ${ }^{94}$, I. Korolkov ${ }^{14}$, E.V. Korolkova ${ }^{149}$, N. Korotkova ${ }^{113}$, O. Kortner ${ }^{115}$, S. Kortner ${ }^{115}$, T. Kosek ${ }^{143}$, V.V. Kostyukhin ${ }^{24}$, A. Kotwal ${ }^{49}$, A. Koulouris ${ }^{10}$, A. Kourkoumeli-Charalampidi ${ }^{70 a, 70 b}$, C. Kourkoumelis ${ }^{9}$, E. Kourlitis ${ }^{149}$, V. Kouskoura ${ }^{29}$, A.B. Kowalewska ${ }^{84}$, R. Kowalewski ${ }^{176}$, C. Kozakai ${ }^{163}$, W. Kozanecki ${ }^{145}$, A.S. Kozhin ${ }^{123}$, V.A. Kramarenko ${ }^{113}$, G. Kramberger ${ }^{91}$, D. Krasnopevtsev ${ }^{60 a}$, M.W. Krasny ${ }^{136}$, A. Krasznahorkay ${ }^{36}$, D. Krauss ${ }^{115}$, J.A. Kremer ${ }^{83 a}$, J. Kretzschmar ${ }^{90}$, P. Krieger ${ }^{167}$,

A. Krishnan ${ }^{61 b}$, K. Krizka ${ }^{18}$, K. Kroeninger ${ }^{47}$, H. Kroha ${ }^{115}$, J. Kroll ${ }^{141}$, J. Kroll ${ }^{137}$, J. Krstic ${ }^{16}$, U. Kruchonak ${ }^{79}$, H. Krüger ${ }^{24}$, N. Krumnack ${ }^{78}$, M.C. Kruse ${ }^{49}$, T. Kubota ${ }^{104}$, S. Kuday ${ }^{4 b}$, J.T. Kuechler ${ }^{46}$, S. Kuehn ${ }^{36}$, A. Kugel ${ }^{61 a}$, T. Kuhl ${ }^{46}$, V. Kukhtin ${ }^{79}$, R. Kukla ${ }^{101}$, Y. Kulchitsky ${ }^{107, a i}$, S. Kuleshov ${ }^{147 b}$, Y.P. Kulinich ${ }^{173}$, M. Kuna ${ }^{58}$, T. Kunigo ${ }^{85}$, A. Kupco ${ }^{141}$, T. Kupfer ${ }^{47}$, O. Kuprash ${ }^{52}$, H. Kurashige ${ }^{82}$, L.L. Kurchaninov ${ }^{168 a}$, Y.A. Kurochkin ${ }^{107}$, A. Kurova ${ }^{112}$, M.G. Kurth ${ }^{15 a, 15 d}$, E.S. Kuwertz ${ }^{36}$, M. Kuze ${ }^{165}$, J. Kvita ${ }^{130}$, T. Kwan ${ }^{103}$, A. La Rosa ${ }^{115}$, J.L. La Rosa Navarro ${ }^{80 d}$, L. La Rotonda ${ }^{41 b, 41 a}$, F. La Ruffa ${ }^{41 b, 41 a}$, C. Lacasta ${ }^{174}$, F. Lacava ${ }^{72 a, 72 b}$, D.P.J. Lack ${ }^{100}$, H. Lacker ${ }^{19}$, D. Lacour ${ }^{136}$, E. Ladygin ${ }^{79}$, R. Lafaye ${ }^{5}$,

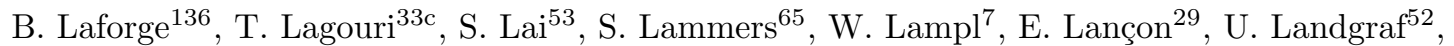
M.P.J. Landon ${ }^{92}$, M.C. Lanfermann ${ }^{54}$, V.S. Lang $^{46}$, J.C. Lange ${ }^{53}$, R.J. Langenberg ${ }^{36}$, A.J. Lankford ${ }^{171}$, F. Lanni ${ }^{29}$, K. Lantzsch ${ }^{24}$, A. Lanza ${ }^{70 a}$, A. Lapertosa ${ }^{55 b, 55 a}$, S. Laplace ${ }^{136}$, J.F. Laporte ${ }^{145}$, T. Lari ${ }^{68 a}$, F. Lasagni Manghi ${ }^{23 b, 23 a}$, M. Lassnig ${ }^{36}$, T.S. Lau ${ }^{63 a}$, A. Laudrain ${ }^{132}$, A. Laurier ${ }^{34}$, M. Lavorgna ${ }^{69 a, 69 b}$, M. Lazzaroni ${ }^{68 a, 68 b}$, B. Le ${ }^{104}$, O. Le Dortz ${ }^{136}$, E. Le Guirriec ${ }^{101}$, M. LeBlanc ${ }^{7}$, T. LeCompte ${ }^{6}$, F. Ledroit-Guillon ${ }^{58}$, C.A. Lee ${ }^{29}$, G.R. Lee ${ }^{147 a}$, L. Lee ${ }^{59}$, S.C. Lee ${ }^{158}$, S.J. Lee ${ }^{34}$, B. Lefebvre ${ }^{168 a}$, M. Lefebvre ${ }^{176}$, F. Legger ${ }^{114}$, C. Leggett ${ }^{18}$, K. Lehmann ${ }^{152}$, N. Lehmann ${ }^{182}$, G. Lehmann Miotto ${ }^{36}$, W.A. Leight ${ }^{46}$, A. Leisos ${ }^{162, v}$, M.A.L. Leite ${ }^{80 d}$, R. Leitner ${ }^{143}$, D. Lellouch ${ }^{180, *}$, K.J.C. Leney ${ }^{42}$, T. Lenz ${ }^{24}$, B. Lenzi ${ }^{36}$,

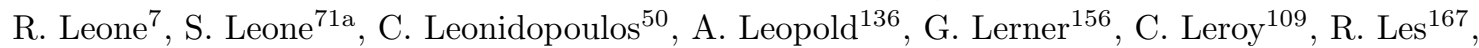
C.G. Lester ${ }^{32}$, M. Levchenko ${ }^{138}$, J. Levêque ${ }^{5}$, D. Levin ${ }^{105}$, L.J. Levinson ${ }^{180}$, D.J. Lewis ${ }^{21}$,

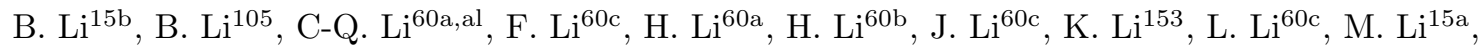

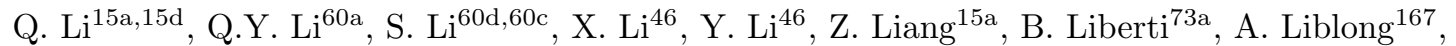
K. Lie ${ }^{63 c}$, S. Liem ${ }^{120}$, C.Y. $\operatorname{Lin}^{32}$, K. Lin ${ }^{106}$, T.H. Lin ${ }^{99}$, R.A. Linck ${ }^{65}$, J.H. Lindon ${ }^{21}$, A.L. Lionti ${ }^{54}$, E. Lipeles ${ }^{137}$, A. Lipniacka ${ }^{17}$, M. Lisovyi ${ }^{61 b}$, T.M. Liss ${ }^{173, \text { as }}$, A. Lister ${ }^{175}$ A.M. Litke ${ }^{146}$, J.D. Little ${ }^{8}$, B. Liu ${ }^{78}$, B.L Liu ${ }^{6}$, H.B. Liu ${ }^{29}$, H. Liu ${ }^{105}$, J.B. Liu ${ }^{60 a}$, J.K.K. Liu ${ }^{135}$,

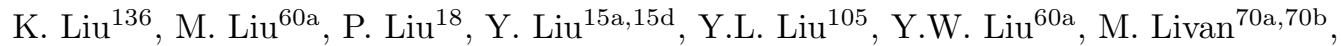
A. Lleres ${ }^{58}$, J. Llorente Merino ${ }^{15 a}$, S.L. Lloyd ${ }^{92}$, C.Y. Lo ${ }^{63 b}$, F. Lo Sterzo ${ }^{42}$, E.M. Lobodzinska ${ }^{46}$, P. Loch ${ }^{7}$, S. Loffredo ${ }^{73 a}, 73 b$, T. Lohse ${ }^{19}$, K. Lohwasser ${ }^{149}$, M. Lokajicek ${ }^{141}$, J.D. Long ${ }^{173}$, R.E. Long ${ }^{89}$, L. Longo ${ }^{36}$, K.A. Looper ${ }^{126}$, J.A. Lopez ${ }^{147 b}$, I. Lopez Paz ${ }^{100}$, A. Lopez Solis ${ }^{149}$, J. Lorenz ${ }^{114}$, N. Lorenzo Martinez ${ }^{5}$, M. Losada ${ }^{22}$, P.J. Lösel ${ }^{114}$, A. Lösle ${ }^{52}$, X. Lou ${ }^{46}$, X. Lou ${ }^{15 a}$,

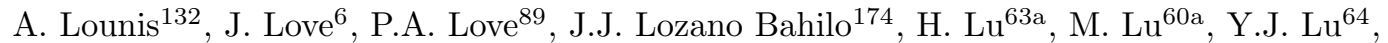
H.J. Lubatti ${ }^{148}$, C. Luci ${ }^{72 a, 72 b}$, A. Lucotte ${ }^{58}$, C. Luedtke ${ }^{52}$, F. Luehring ${ }^{65}$, I. Luise ${ }^{136}$, L. Luminari ${ }^{72 a}$, B. Lund-Jensen ${ }^{154}$, M.S. Lutz ${ }^{102}$, D. Lynn ${ }^{29}$, R. Lysak ${ }^{141}$, E. Lytken ${ }^{96}$, F. Lyu ${ }^{15 a}$, V. Lyubushkin ${ }^{79}$, T. Lyubushkina ${ }^{79}$, H. $\mathrm{Ma}^{29}$, L.L. Ma ${ }^{60 b}$, Y. Ma ${ }^{60 b}$, G. Maccarrone ${ }^{51}$, A. Macchiolo ${ }^{115}$, C.M. Macdonald ${ }^{149}$, J. Machado Miguens ${ }^{137,140 b}$, D. Madaffari ${ }^{174}$, R. Madar ${ }^{38}$, W.F. Mader ${ }^{48}$, N. Madysa ${ }^{48}$, J. Maeda ${ }^{82}$, K. Maekawa ${ }^{163}$, S. Maeland ${ }^{17}$, T. Maeno ${ }^{29}$, M. Maerker ${ }^{48}$, A.S. Maevskiy ${ }^{113}$, V. Magerl ${ }^{52}$, N. Magini ${ }^{78}$, D.J. Mahon ${ }^{39}$, C. Maidantchik ${ }^{80 b}$, T. Maier ${ }^{114}$, A. Maio140a,140b,140d, O. Majersky ${ }^{28 a}$, S. Majewski ${ }^{131}$, Y. Makida ${ }^{81}$, N. Makovec ${ }^{132}$, B. Malaescu ${ }^{136}$, Pa. Malecki ${ }^{84}$, V.P. Maleev ${ }^{138}$, F. Malek ${ }^{58}$, U. Mallik ${ }^{77}$, D. Malon ${ }^{6}$, C. Malone ${ }^{32}$, S. Maltezos ${ }^{10}$, S. Malyukov ${ }^{36}$, J. Mamuzic ${ }^{174}$, G. Mancini ${ }^{51}$, I. Mandić ${ }^{91}$,

L. Manhaes de Andrade Filho ${ }^{80 a}$, I.M. Maniatis ${ }^{162}$, J. Manjarres Ramos ${ }^{48}$, K.H. Mankinen ${ }^{96}$, A. Mann $^{114}$, A. Manousos ${ }^{76}$, B. Mansoulie ${ }^{145}$, I. Manthos ${ }^{162}$, S. Manzoni ${ }^{120}$, A. Marantis ${ }^{162}$, G. Marceca ${ }^{30}$, L. Marchese ${ }^{135}$, G. Marchiori ${ }^{136}$, M. Marcisovsky ${ }^{141}$, C. Marcon ${ }^{96}$, 
C.A. Marin Tobon ${ }^{36}$, M. Marjanovic ${ }^{38}$, F. Marroquim ${ }^{80 b}$, Z. Marshall ${ }^{18}$, M.U.F Martensson ${ }^{172}$, S. Marti-Garcia ${ }^{174}$, C.B. Martin ${ }^{126}$, T.A. Martin ${ }^{178}$, V.J. Martin ${ }^{50}$, B. Martin dit Latour ${ }^{17}$, M. Martinez ${ }^{14, x}$, V.I. Martinez Outschoorn ${ }^{102}$, S. Martin-Haugh ${ }^{144}$, V.S. Martoiu ${ }^{27 b}$, A.C. Martyniuk ${ }^{94}$, A. Marzin ${ }^{36}$, L. Masetti ${ }^{99}$, T. Mashimo ${ }^{163}$, R. Mashinistov ${ }^{110}$, J. Masik ${ }^{100}$, A.L. Maslennikov ${ }^{122 b, 122 a}$, L.H. Mason ${ }^{104}$, L. Massa ${ }^{73 a, 73 b}$, P. Massarotti ${ }^{69 a, 69 b}$, P. Mastrandrea ${ }^{71 a, 71 b}$, A. Mastroberardino ${ }^{41 b, 41 a}$, T. Masubuchi ${ }^{163}$, A. Matic ${ }^{114}$, P. Mättig ${ }^{24}$, J. Maurer ${ }^{27 b}$, B. Maček ${ }^{91}$, S.J. Maxfield ${ }^{90}$, D.A. Maximov ${ }^{122 b, 122 a}$, R. Mazini ${ }^{158}$, I. Maznas ${ }^{162}$, S.M. Mazza ${ }^{146}$, S.P. Mc Kee ${ }^{105}$, T.G. McCarthy ${ }^{115}$, L.I. McClymont ${ }^{94}$, W.P. McCormack ${ }^{18}$, E.F. McDonald ${ }^{104}$, J.A. Mcfayden ${ }^{36}$, M.A. McKay ${ }^{42}$, K.D. McLean ${ }^{176}$, S.J. McMahon ${ }^{144}$, P.C. $\mathrm{McNamara}^{104}$, C.J. $\mathrm{McNicol}^{178}$, R.A. $\mathrm{McPherson}^{176, \text { ac }}$, J.E. Mdhluli ${ }^{33 c}$, Z.A. Meadows ${ }^{102}$, S. Meehan ${ }^{148}$, T. Megy ${ }^{52}$, S. Mehlhase ${ }^{114}$, A. Mehta ${ }^{90}$, T. Meideck ${ }^{58}$, B. Meirose ${ }^{43}$, D. Melini ${ }^{174}$, B.R. Mellado Garcia ${ }^{33 c}$, J.D. Mellenthin ${ }^{53}$, M. Melo ${ }^{28 a}$, F. Meloni ${ }^{46}$, A. Melzer $^{24}$, S.B. Menary ${ }^{100}$, E.D. Mendes Gouveia ${ }^{140 a, 140 e}$, L. Meng $^{36}$, X.T. Meng ${ }^{105}$, S. Menke ${ }^{115}$, E. Meoni ${ }^{41 b, 41 a}$,

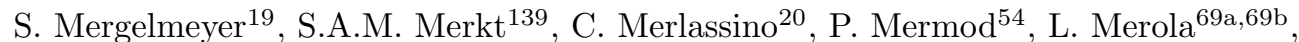
C. Meroni ${ }^{68 a}$, O. Meshkov ${ }^{113}$, J.K.R. Meshreki ${ }^{151}$, A. Messina ${ }^{72 a, 72 b}$, J. Metcalfe $^{6}$, A.S. Mete ${ }^{171}$, C. Meyer ${ }^{65}$, J. Meyer ${ }^{160}$, J-P. Meyer ${ }^{145}$, H. Meyer Zu Theenhausen ${ }^{61 a}$, F. Miano ${ }^{156}$, R.P. Middleton ${ }^{144}$, L. Mijović ${ }^{50}$, G. Mikenberg ${ }^{180}$, M. Mikestikova ${ }^{141}$, M. Mikuž ${ }^{91}$, H. Mildner ${ }^{149}$, M. Milesi ${ }^{104}$, A. Milic ${ }^{167}$, D.A. Millar ${ }^{92}$, D.W. Miller ${ }^{37}$, A. Milov ${ }^{180}$, D.A. Milstead ${ }^{45 a, 45 b}$, R.A. Mina ${ }^{153, p}$, A.A. Minaenko ${ }^{123}$, M. Miñano Moya ${ }^{174}$, I.A. Minashvili ${ }^{159 b}$, A.I. Mincer ${ }^{124}$, B. Mindur ${ }^{83 a}$, M. Mineev ${ }^{79}$, Y. Minegishi ${ }^{163}$, Y. Ming ${ }^{181}$, L.M. Mir ${ }^{14}$, A. Mirto ${ }^{67 a, 67 b}$, K.P. Mistry ${ }^{137}$, T. Mitani ${ }^{179}$, J. Mitrevski ${ }^{114}$, V.A. Mitsou ${ }^{174}$, M. Mittal ${ }^{60 c}$, A. Miucci ${ }^{20}$, P.S. Miyagawa ${ }^{149}$, A. Mizukami ${ }^{81}$, J.U. Mjörnmark ${ }^{96}$, T. Mkrtchyan $^{184}$, M. Mlynarikova ${ }^{143}$, T. Moa ${ }^{45 a, 45 b}$, K. Mochizuki ${ }^{109}$, P. Mogg ${ }^{52}$, S. Mohapatra ${ }^{39}$, R. Moles-Valls ${ }^{24}$, M.C. Mondragon ${ }^{106}$, K. Mönig ${ }^{46}$, J. Monk ${ }^{40}$, E. Monnier ${ }^{101}$, A. Montalbano ${ }^{152}$, J. Montejo Berlingen ${ }^{36}$, M. Montella ${ }^{94}$, F. Monticelli ${ }^{88}$, S. Monzani ${ }^{68 a}$, N. Morange ${ }^{132}$, D. Moreno ${ }^{22}$, M. Moreno Llácer ${ }^{36}$, P. Morettini ${ }^{55 b}$, M. Morgenstern ${ }^{120}$, S. Morgenstern ${ }^{48}$, D. Mori ${ }^{152}$, M. Morii ${ }^{59}$, M. Morinaga ${ }^{179}$, V. Morisbak ${ }^{134}$, A.K. Morley ${ }^{36}$, G. Mornacchi ${ }^{36}$, A.P. Morris ${ }^{94}$, L. Morvaj ${ }^{155}$, P. Moschovakos ${ }^{10}$, B. Moser ${ }^{120}$, M. Mosidze ${ }^{159 b}$, H.J. Moss ${ }^{149}$, J. Moss ${ }^{31, m}$, K. Motohashi ${ }^{165}$, E. Mountricha ${ }^{36}$, E.J.W. Moyse ${ }^{102}$, S. Muanza ${ }^{101}$, F. Mueller ${ }^{115}$, J. Mueller ${ }^{139}$, R.S.P. Mueller ${ }^{114}$, D. Muenstermann ${ }^{89}$, G.A. Mullier ${ }^{96}$, J.L. Munoz Martinez ${ }^{14}$, F.J. Munoz Sanchez ${ }^{100}$, P. Murin ${ }^{28 b}$, W.J. Murray ${ }^{178,144}$, A. Murrone ${ }^{68 a, 68 b}$, M. Muškinja ${ }^{18}$, C. Mwewa ${ }^{33 a}$, A.G. Myagkov ${ }^{123, \text { an }}$, J. Myers ${ }^{131}$, M. Myska ${ }^{142}$, B.P. Nachman ${ }^{18}$,

O. Nackenhorst ${ }^{47}$, K. Nagai ${ }^{135}$, K. Nagano ${ }^{81}$, Y. Nagasaka ${ }^{62}$, M. Nagel ${ }^{52}$, E. Nagy ${ }^{101}$, A.M. Nairz ${ }^{36}$, Y. Nakahama ${ }^{117}$, K. Nakamura ${ }^{81}$, T. Nakamura ${ }^{163}$, I. Nakano ${ }^{127}$, H. Nanjo ${ }^{133}$, F. Napolitano ${ }^{61 a}$, R.F. Naranjo Garcia ${ }^{46}$, R. Narayan $^{11}$, D.I. Narrias Villar ${ }^{61 a}$, I. Naryshkin ${ }^{138}$, T. Naumann ${ }^{46}$, G. Navarro ${ }^{22}$, H.A. Neal ${ }^{105, *}$, P.Y. Nechaeva ${ }^{110}$, F. Nechansky ${ }^{46}$, T.J. Neep ${ }^{145}$, A. Negri ${ }^{70 a, 70 b}$, M. Negrini ${ }^{23 b}$, S. Nektarijevic ${ }^{119}$, C. Nellist ${ }^{53}$, M.E. Nelson ${ }^{135}$, S. Nemecek ${ }^{141}$, P. Nemethy ${ }^{124}$, M. Nessi ${ }^{36, d}$, M.S. Neubauer ${ }^{173}$, M. Neumann ${ }^{182}$, P.R. Newman ${ }^{21}$, T.Y. $\mathrm{Ng}^{63 c}$, Y.S. $\mathrm{Ng}^{19}$, Y.W.Y. $\mathrm{Ng}^{171}$, H.D.N. Nguyen ${ }^{101}$, T. Nguyen Manh ${ }^{109}$, E. Nibigira ${ }^{38}$, R.B. Nickerson ${ }^{135}$, R. Nicolaidou ${ }^{145}$, D.S. Nielsen ${ }^{40}$, J. Nielsen $^{146}$, N. Nikiforou ${ }^{11}$, V. Nikolaenko ${ }^{123, \text { an }}$, I. Nikolic-Audit ${ }^{136}$, K. Nikolopoulos ${ }^{21}$, P. Nilsson ${ }^{29}$, H.R. Nindhito ${ }^{54}$,

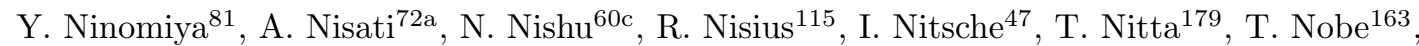
Y. Noguchi ${ }^{85}$, M. Nomachi ${ }^{133}$, I. Nomidis ${ }^{136}$, M.A. Nomura ${ }^{29}$, M. Nordberg ${ }^{36}$,

N. Norjoharuddeen ${ }^{135}$, T. Novak ${ }^{91}$, O. Novgorodova ${ }^{48}$, R. Novotny ${ }^{142}$, L. Nozka $^{130}$, K. Ntekas ${ }^{171}$, E. Nurse ${ }^{94}$, F. Nuti ${ }^{104}$, F.G. Oakham ${ }^{34, \text { av }}$, H. Oberlack ${ }^{115}$, J. Ocariz ${ }^{136}$, A. Ochi ${ }^{82}$, I. Ochoa ${ }^{39}$, J.P. Ochoa-Ricoux ${ }^{147 a}$, K. O'Connor ${ }^{26}$, S. Oda ${ }^{87}$, S. Odaka ${ }^{81}$, S. Oerdek ${ }^{53}$, A. Ogrodnik ${ }^{83 a}$, A. $\mathrm{Oh}^{100}$, S.H. $\mathrm{Oh}^{49}$, C.C. $\mathrm{Ohm}^{154}$, H. Oide ${ }^{55 b, 55 a}$, M.L. Ojeda ${ }^{167}$, H. Okawa ${ }^{169}$, Y. Okazaki ${ }^{85}$, Y. Okumura ${ }^{163}$, T. Okuyama ${ }^{81}$, A. Olariu ${ }^{27 b}$, L.F. Oleiro Seabra ${ }^{140 a}$, S.A. Olivares Pino ${ }^{147 a}$, 
D. Oliveira Damazio ${ }^{29}$, J.L. Oliver ${ }^{1}$, M.J.R. Olsson ${ }^{171}$, A. Olszewski ${ }^{84}$, J. Olszowska $^{84}$, D.C. O'Neil ${ }^{152}$, A. Onofre ${ }^{140 a, 140 e}$, K. Onogi ${ }^{117}$, P.U.E. Onyisi ${ }^{11}$, H. Oppen ${ }^{134}$, M.J. Oreglia ${ }^{37}$, G.E. Orellana ${ }^{88}$, Y. Oren ${ }^{161}$, D. Orestano ${ }^{74 a}, 74 b$, N. Orlando ${ }^{14}$, R.S. Orr ${ }^{167}$, B. Osculati ${ }^{55 b, 55 a, *}$, V. O'Shea ${ }^{57}$, R. Ospanov ${ }^{60 a}$, G. Otero y Garzon ${ }^{30}$, H. Otono ${ }^{87}$, M. Ouchrif ${ }^{35 d}$, F. Ould-Saada ${ }^{134}$, A. Ouraou ${ }^{145}$, Q. Ouyang ${ }^{15 a}$, M. Owen ${ }^{57}$, R.E. Owen ${ }^{21}$, V.E. Ozcan ${ }^{12 c}$, N. Ozturk ${ }^{8}$, J. Pacalt ${ }^{130}$, H.A. Pacey ${ }^{32}$, K. Pachal ${ }^{49}$, A. Pacheco Pages ${ }^{14}$, C. Padilla Aranda ${ }^{14}$, S. Pagan Griso ${ }^{18}$, M. Paganini ${ }^{183}$, G. Palacino ${ }^{65}$, S. Palazzo ${ }^{50}$, S. Palestini ${ }^{36}$, M. Palka ${ }^{83 b}$, D. Pallin ${ }^{38}$, I. Panagoulias ${ }^{10}$, C.E. Pandini ${ }^{36}$, J.G. Panduro Vazquez ${ }^{93}$, P. Pani ${ }^{46}$, G. Panizzo ${ }^{66 a, 66 c}$, L. Paolozzi ${ }^{54}$, C. Papadatos ${ }^{109}$, K. Papageorgiou ${ }^{9, h}$, A. Paramonov ${ }^{6}$, D. Paredes Hernandez ${ }^{63 b}$, S.R. Paredes Saenz ${ }^{135}$, B. Parida ${ }^{166}$, T.H. Park ${ }^{167}$, A.J. Parker ${ }^{89}$, M.A. Parker ${ }^{32}$, F. Parodi ${ }^{55 b, 55 a,}$ E.W.P. Parrish ${ }^{121}$, J.A. Parsons ${ }^{39}$, U. Parzefall ${ }^{52}$, L. Pascual Dominguez ${ }^{136}$, V.R. Pascuzzi ${ }^{167}$, J.M.P. Pasner ${ }^{146}$, E. Pasqualucci ${ }^{72 a}$, S. Passaggio ${ }^{55 b}$, F. Pastore ${ }^{93}$, P. Pasuwan ${ }^{45 a, 45 b}$, S. Pataraia ${ }^{99}$, J.R. Pater ${ }^{100}$, A. Pathak ${ }^{181}$, T. Pauly ${ }^{36}$, B. Pearson ${ }^{115}$, M. Pedersen ${ }^{134}$, L. Pedraza Diaz ${ }^{119}$, R. Pedro ${ }^{140 a, 140 b}$, S.V. Peleganchuk ${ }^{122 b, 122 a}$, O. Penc ${ }^{141}$, C. Peng ${ }^{15 a}$, H. Peng ${ }^{60 a}$, B.S. Peralva ${ }^{80 a}$, M.M. Perego ${ }^{132}$, A.P. Pereira Peixoto ${ }^{140 a, 140 e}$, D.V. Perepelitsa ${ }^{29}$, F. Peri ${ }^{19}$, L. Perini ${ }^{68 a, 68 b}$, H. Pernegger ${ }^{36}$, S. Perrella ${ }^{69 a, 69 b}$, V.D. Peshekhonov ${ }^{79, *}$, K. Peters ${ }^{46}$, R.F.Y. Peters ${ }^{100}$, B.A. Petersen ${ }^{36}$, T.C. Petersen ${ }^{40}$, E. Petit ${ }^{58}$, A. Petridis ${ }^{1}$, C. Petridou ${ }^{162}$, P. Petroff ${ }^{132}$, M. Petrov ${ }^{135}$, F. Petrucci ${ }^{74 a, 74 b}$, M. Pettee ${ }^{183}$, N.E. Pettersson ${ }^{102}$, K. Petukhova ${ }^{143}$, A. Peyaud ${ }^{145}$, R. Pezoa ${ }^{147 b}$, T. Pham ${ }^{104}$, F.H. Phillips ${ }^{106}$, P.W. Phillips ${ }^{144}$, M.W. Phipps ${ }^{173}$, G. Piacquadio ${ }^{155}$, E. Pianori ${ }^{18}$, A. Picazio ${ }^{102}$, R.H. Pickles ${ }^{100}$, R. Piegaia ${ }^{30}$, D. Pietreanu ${ }^{27 b}$, J.E. Pilcher ${ }^{37}$, A.D. Pilkington ${ }^{100}$, M. Pinamonti ${ }^{73 a, 73 b}$, J.L. Pinfold ${ }^{3}$, M. Pitt ${ }^{180}$, L. Pizzimento ${ }^{73 a, 73 b}$, M.-A. Pleier ${ }^{29}$, V. Pleskot ${ }^{143}$, E. Plotnikova ${ }^{79}$, D. Pluth ${ }^{78}$, P. Podberezko ${ }^{122 b, 122 a}$, R. Poettgen ${ }^{96}$, R. Poggi ${ }^{54}$, L. Poggioli ${ }^{132}$, I. Pogrebnyak ${ }^{106}$, D. Pohl $^{24}$, I. Pokharel ${ }^{53}$, G. Polesello ${ }^{70 a}$, A. Poley ${ }^{18}$, A. Policicchio ${ }^{72 a, 72 b}$, R. Polifka ${ }^{36}$, A. Polini2 ${ }^{23 b}$, C.S. Pollard ${ }^{46}$, V. Polychronakos ${ }^{29}$, D. Ponomarenko ${ }^{112}$, L. Pontecorvo ${ }^{36}$, S. Popa ${ }^{27 a}$, G.A. Popeneciu ${ }^{27 d}$, D.M. Portillo Quintero ${ }^{136}$, S. Pospisil ${ }^{142}$, K. Potamianos ${ }^{46}$, I.N. Potrap ${ }^{79}$, C.J. Potter ${ }^{32}$, H. Potti ${ }^{11}$, T. Poulsen ${ }^{96}$, J. Poveda ${ }^{36}$, T.D. Powell ${ }^{149}$, M.E. Pozo Astigarraga ${ }^{36}$, P. Pralavorio ${ }^{101}$, S. Prell ${ }^{78}$, D. Price ${ }^{100}$, M. Primavera ${ }^{67 a}$, S. Prince ${ }^{103}$, M.L. Proffitt ${ }^{148}$, N. Proklova ${ }^{112}$, K. Prokofiev ${ }^{63 c}$, F. Prokoshin ${ }^{147 b}$, S. Protopopescu ${ }^{29}$, J. Proudfoot ${ }^{6}$, M. Przybycien ${ }^{83 a}$, A. Puri ${ }^{173}$, P. Puzo ${ }^{132}$, J. Qian ${ }^{105}$, Y. Qin ${ }^{100}$, A. Quadt ${ }^{53}$, M. Queitsch-Maitland ${ }^{46}$, A. Qureshi ${ }^{1}$, P. Rados ${ }^{104}$, F. Ragusa ${ }^{68 a, 68 b}$, G. Rahal ${ }^{97}$, J.A. Raine ${ }^{54}$, S. Rajagopalan ${ }^{29}$, A. Ramirez Morales ${ }^{92}$, K. Ran ${ }^{15 a, 15 d}$, T. Rashid ${ }^{132}$, S. Raspopov ${ }^{5}$, M.G. Ratti ${ }^{68 a, 68 b}$, D.M. Rauch ${ }^{46}$, F. Rauscher ${ }^{114}$, S. Rave ${ }^{99}$, B. Ravina ${ }^{149}$, I. Ravinovich ${ }^{180}$, J.H. Rawling ${ }^{100}$, M. Raymond ${ }^{36}$, A.L. Read ${ }^{134}$, N.P. Readioff ${ }^{58}$, M. Reale ${ }^{67 a, 67 b}$, D.M. Rebuzzi ${ }^{70 a}, 70 b$, A. Redelbach ${ }^{177}$, G. Redlinger ${ }^{29}$, R.G. Reed ${ }^{33 c}$, K. Reeves ${ }^{43}$, L. Rehnisch $^{19}$, J. Reichert ${ }^{137}$, D. Reikher ${ }^{161}$, A. Reiss ${ }^{99}$, A. Rej ${ }^{151}$, C. Rembser ${ }^{36}$, H. Ren ${ }^{15 a}$, M. Rescigno ${ }^{72 a}$, S. Resconi ${ }^{68 a}$, E.D. Resseguie ${ }^{137}$, S. Rettie ${ }^{175}$, E. Reynolds ${ }^{21}$, O.L. Rezanova ${ }^{122 b, 122 a}$, P. Reznicek ${ }^{143}$, E. Ricci ${ }^{75 a, 75 b}$, R. Richter ${ }^{115}$, S. Richter ${ }^{46}$, E. Richter-Was ${ }^{83 b}$, O. Ricken ${ }^{24}$, M. Ridel ${ }^{136}$, P. Rieck ${ }^{115}$, C.J. Riegel ${ }^{182}$, O. Rifki ${ }^{46}$, M. Rijssenbeek ${ }^{155}$, A. Rimoldi ${ }^{70 a, 70 b}$,

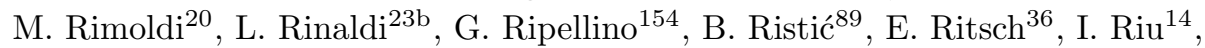
J.C. Rivera Vergara ${ }^{147 a}$, F. Rizatdinova ${ }^{129}$, E. Rizvi ${ }^{92}$, C. Rizzi ${ }^{36}$, R.T. Roberts ${ }^{100}$, S.H. Robertson ${ }^{103, \text { ac }}$, D. Robinson ${ }^{32}$, J.E.M. Robinson ${ }^{46}$, A. Robson ${ }^{57}$, E. Rocco ${ }^{99}$, C. Roda ${ }^{71 a, 71 b}$, Y. Rodina ${ }^{101}$, S. Rodriguez Bosca ${ }^{174}$, A. Rodriguez Perez ${ }^{14}$, D. Rodriguez Rodriguez ${ }^{174}$, A.M. Rodríguez Vera ${ }^{168 \mathrm{~b}}$, S. Roe ${ }^{36}$, O. Røhne ${ }^{134}$, R. Röhrig ${ }^{115}$, C.P.A. Roland ${ }^{65}$, J. Roloff ${ }^{59}$, A. Romaniouk ${ }^{112}$, M. Romano ${ }^{23 b, 23 a}$, N. Rompotis ${ }^{90}$, M. Ronzani $^{124}$, L. Roos ${ }^{136}$, S. Rosati ${ }^{72 a}$, K. Rosbach ${ }^{52}$, N-A. Rosien ${ }^{53}$, G. Rosin ${ }^{102}$, B.J. Rosser ${ }^{137}$, E. Rossi ${ }^{46}$, E. Rossi ${ }^{74 a, 74 b}$, E. Rossi ${ }^{69 a}$ a69b , L.P. Rossi ${ }^{55 b}$, L. Rossini ${ }^{68 a, 68 b}$, J.H.N. Rosten ${ }^{32}$, R. Rosten ${ }^{14}$, M. Rotaru ${ }^{27 b}$, J. Rothberg ${ }^{148}$, D. Rousseau ${ }^{132}$, D. Roy ${ }^{33 c}$, A. Rozanov $^{101}$, Y. Rozen ${ }^{160}$, 
X. Ruan ${ }^{33 c}$, F. Rubbo ${ }^{153}$, F. Rühr ${ }^{52}$, A. Ruiz-Martinez ${ }^{174}$, A. Rummler ${ }^{36}$, Z. Rurikova ${ }^{52}$, N.A. Rusakovich ${ }^{79}$, H.L. Russell ${ }^{103}$, L. Rustige ${ }^{38,47}$, J.P. Rutherfoord ${ }^{7}$, E.M. Rüttinger ${ }^{46, j}$, Y.F. Ryabov ${ }^{138}$, M. Rybar ${ }^{39}$, G. Rybkin ${ }^{132}$, S. Ryu ${ }^{6}$, A. Ryzhov ${ }^{123}$, G.F. Rzehorz ${ }^{53}$, P. Sabatini ${ }^{53}$, G. Sabato ${ }^{120}$, S. Sacerdoti ${ }^{132}$, H.F-W. Sadrozinski ${ }^{146}$, R. Sadykov ${ }^{79}$, F. Safai Tehrani ${ }^{72 a}$, P. Saha ${ }^{121}$, S. Saha ${ }^{103}$, M. Sahinsoy ${ }^{61 a}$, A. Sahu ${ }^{182}$, M. Saimpert ${ }^{46}$, M. Saito ${ }^{163}$, T. Saito ${ }^{163}$, H. Sakamoto ${ }^{163}$, A. Sakharov ${ }^{124, a m}$, D. Salamani ${ }^{54}$, G. Salamanna ${ }^{74 a, 74 b}$, J.E. Salazar Loyola ${ }^{147 b}$, P.H. Sales De Bruin ${ }^{172}$, D. Salihagic ${ }^{115, *}$, A. Salnikov ${ }^{153}$, J. Salt ${ }^{174}$, D. Salvatore ${ }^{41 b, 41 a}$, F. Salvatore ${ }^{156}$, A. Salvucci6ia,63b,63c , A. Salzburger ${ }^{36}$, J. Samarati ${ }^{36}$,

D. Sammel ${ }^{52}$, D. Sampsonidis ${ }^{162}$, D. Sampsonidou ${ }^{162}$, J. Sánchez ${ }^{174}$, A. Sanchez Pineda ${ }^{66 a, 66 c}$, H. Sandaker ${ }^{134}$, C.O. Sander ${ }^{46}$, M. Sandhoff ${ }^{182}$, C. Sandoval $^{22}$, D.P.C. Sankey ${ }^{144}$, M. Sannino ${ }^{55 b, 55 a}$, Y. Sano ${ }^{117}$, A. Sansoni ${ }^{51}$, C. Santoni ${ }^{38}$, H. Santos ${ }^{140 a, 140 b}$, S.N. Santpur ${ }^{18}$, A. Santra ${ }^{174}$, A. Sapronov ${ }^{79}$, J.G. Saraiva ${ }^{140 a, 140 d}$, O. Sasaki ${ }^{81}$, K. Sato ${ }^{169}$, E. Sauvan ${ }^{5}$, P. Savard ${ }^{167, a v}$, N. Savic ${ }^{115}$, R. Sawada ${ }^{163}$, C. Sawyer ${ }^{144}$, L. Sawyer ${ }^{95, a k}$, C. Sbarra ${ }^{23 b}$, A. Sbrizzi ${ }^{23 a}$, T. Scanlon ${ }^{94}$, J. Schaarschmidt ${ }^{148}$, P. Schacht ${ }^{115}$, B.M. Schachtner ${ }^{114}$, D. Schaefer ${ }^{37}$, L. Schaefer ${ }^{137}$, J. Schaeffer ${ }^{99}$, S. Schaepe ${ }^{36}$, U. Schäfer ${ }^{99}$, A.C. Schaffer ${ }^{132}$, D. Schaile ${ }^{114}$, R.D. Schamberger ${ }^{155}$, N. Scharmberg ${ }^{100}$, V.A. Schegelsky ${ }^{138}$, D. Scheirich ${ }^{143}$, F. Schenck ${ }^{19}$, M. Schernau ${ }^{171}$, C. Schiavi ${ }^{55 b, 55 a}$, S. Schier ${ }^{146}$, L.K. Schildgen ${ }^{24}$, Z.M. Schillaci ${ }^{26}$, E.J. Schioppa ${ }^{36}$, M. Schioppa ${ }^{41 b, 41 a}$, K.E. Schleicher ${ }^{52}$, S. Schlenker ${ }^{36}$,

K.R. Schmidt-Sommerfeld ${ }^{115}$, K. Schmieden ${ }^{36}$, C. Schmitt ${ }^{99}$, S. Schmitt ${ }^{46}$, S. Schmitz ${ }^{99}$, J.C. Schmoeckel ${ }^{46}$, U. Schnoor ${ }^{52}$, L. Schoeffel ${ }^{145}$, A. Schoening61b, E. Schopf ${ }^{135}$, M. Schott ${ }^{99}$, J.F.P. Schouwenberg ${ }^{119}$, J. Schovancova ${ }^{36}$, S. Schramm ${ }^{54}$, A. Schulte ${ }^{99}$, H-C. Schultz-Coulon ${ }^{61 a}$, M. Schumacher ${ }^{52}$, B.A. Schumm ${ }^{146}$, Ph. Schune ${ }^{145}$, A. Schwartzman ${ }^{153}$, T.A. Schwarz ${ }^{105}$, Ph. Schwemling ${ }^{145}$, R. Schwienhorst ${ }^{106}$, A. Sciandra ${ }^{24}$, G. Sciolla ${ }^{26}$, M. Scornajenghi ${ }^{41 b, 41 a}$, F. Scuri ${ }^{71 a}$, F. Scutti ${ }^{104}$, L.M. Scyboz ${ }^{115}$, C.D. Sebastiani ${ }^{72 a, 72 b}$, P. Seema ${ }^{19}$, S.C. Seidel ${ }^{118}$, A. Seiden ${ }^{146}$, T. Seiss ${ }^{37}$, J.M. Seixas ${ }^{80 b}$, G. Sekhniaidze ${ }^{69 a}$, K. Sekhon ${ }^{105}$, S.J. Sekula ${ }^{42}$, N. Semprini-Cesari ${ }^{23 b, 23 a}$, S. Sen ${ }^{49}$, S. Senkin ${ }^{38}$, C. Serfon ${ }^{76}$, L. Serin ${ }^{132}$, L. Serkin ${ }^{66 a, 66 b}$, M. Sessa ${ }^{60 a}$, H. Severini ${ }^{128}$, F. Sforza ${ }^{170}$, A. Sfyrla ${ }^{54}$, E. Shabalina ${ }^{53}$, J.D. Shahinian ${ }^{146}$, N.W. Shaikh ${ }^{45 a, 45 b}$, D. Shaked Renous ${ }^{180}$, L.Y. Shan ${ }^{15 a}$, R. Shang ${ }^{173}$, J.T. Shank ${ }^{25}$, M. Shapiro ${ }^{18}$, A.S. Sharma ${ }^{1}$, A. Sharma ${ }^{135}$, P.B. Shatalov ${ }^{111}$, K. Shaw ${ }^{156}$, S.M. Shaw ${ }^{100,}$ A. Shcherbakova ${ }^{138}$, Y. Shen ${ }^{128}$, N. Sherafati ${ }^{34}$, A.D. Sherman ${ }^{25}$, P. Sherwood ${ }^{94}$, L. Shi ${ }^{158, a r}$, S. Shimizu ${ }^{81}$, C.O. Shimmin ${ }^{183}$, Y. Shimogama ${ }^{179}$, M. Shimojima ${ }^{116}$, I.P.J. Shipsey ${ }^{135}$, S. Shirabe ${ }^{87}$, M. Shiyakova ${ }^{79}$,aa, J. Shlomi ${ }^{180}$, A. Shmeleva ${ }^{110}$, M.J. Shochet ${ }^{37}$, S. Shojaii ${ }^{104}$, D.R. Shope ${ }^{128}$, S. Shrestha ${ }^{126}$, E. Shulga ${ }^{180}$, P. Sicho ${ }^{141}$, A.M. Sickles ${ }^{173}$, P.E. Sidebo ${ }^{154}$, E. Sideras Haddad ${ }^{33 c}$, O. Sidiropoulou ${ }^{36}$, A. Sidoti ${ }^{23 b, 23 a}$, F. Siegert ${ }^{48}$, Dj. Sijacki ${ }^{16}$, M. Silva Jr. ${ }^{181}$, M.V. Silva Oliveira ${ }^{80 a}$, S.B. Silverstein ${ }^{45 a}$, S. Simion ${ }^{132}$, E. Simioni ${ }^{99}$, M. Simon ${ }^{99}$, R. Simoniello ${ }^{99}$, P. Sinervo ${ }^{167}$, N.B. Sinev ${ }^{131}$, M. Sioli ${ }^{23 b}$,23a, I. Siral ${ }^{105}$, S.Yu. Sivoklokov ${ }^{113}$, J. Sjölin ${ }^{45 a, 45 b}$, E. Skorda ${ }^{96}$, P. Skubic ${ }^{128}$, M. Slawinska ${ }^{84}$, K. Sliwa ${ }^{170}$, R. Slovak ${ }^{143}$, V. Smakhtin ${ }^{180}$, B.H. Smart ${ }^{144}$, J. Smiesko ${ }^{28 a}$, N. Smirnov ${ }^{112}$, S.Yu. Smirnov ${ }^{112}$, Y. Smirnov ${ }^{112}$, L.N. Smirnova ${ }^{113, s}$, O. Smirnova ${ }^{96}$, J.W. Smith ${ }^{53}$, M. Smizanska ${ }^{89}$, K. Smolek ${ }^{142}$, A. Smykiewicz ${ }^{84}$, A.A. Snesarev ${ }^{110}$, I.M. Snyder ${ }^{131}$, S. Snyder ${ }^{29}$, R. Sobie ${ }^{176, a c}$, A.M. Soffa ${ }^{171}$, A. Soffer ${ }^{161}$, A. Søgaard ${ }^{50}$, F. Sohns ${ }^{53}$, G. Sokhrannyi ${ }^{91}$, C.A. Solans Sanchez ${ }^{36}$, E.Yu. Soldatov ${ }^{112}$, U. Soldevila ${ }^{174}$, A.A. Solodkov ${ }^{123}$, A. Soloshenko ${ }^{79}$, O.V. Solovyanov ${ }^{123}$, V. Solovyev ${ }^{138}$, P. Sommer ${ }^{149}$, H. Son ${ }^{170}$, W. Song ${ }^{144}$, W.Y. Song ${ }^{168 b}$, A. Sopczak ${ }^{142}$, F. Sopkova ${ }^{28 b}$, C.L. Sotiropoulou ${ }^{71 a, 71 b}$, S. Sottocornola ${ }^{70 a, 70 b}$, R. Soualah ${ }^{66 a, 66 c, g}$, A.M. Soukharev ${ }^{122 b, 122 a}$, D. South ${ }^{46}$, S. Spagnolo ${ }^{67 a, 67 b}$, M. Spalla ${ }^{115}$, M. Spangenberg ${ }^{178}$, F. Spanò ${ }^{93}$, D. Sperlich ${ }^{19}$, T.M. Spieker ${ }^{61 a}$, R. Spighi ${ }^{23 b}$, G. Spigo ${ }^{36}$, L.A. Spiller ${ }^{104}$, M. Spina ${ }^{156}$, D.P. Spiteri ${ }^{57}$, M. Spousta ${ }^{143}$, A. Stabile ${ }^{68 a, 68 b}$, B.L. Stamas ${ }^{121}$, R. Stamen ${ }^{61 a}$, M. Stamenkovic ${ }^{120}$, S. Stamm ${ }^{19}$, E. Stanecka ${ }^{84}$, R.W. Stanek ${ }^{6}$, B. Stanislaus ${ }^{135}$, 
M.M. Stanitzki ${ }^{46}$, M. Stankaityte ${ }^{135}$, B. Stapf ${ }^{120}$, E.A. Starchenko ${ }^{123}$, G.H. Stark ${ }^{146}$, J. $\operatorname{Stark}^{58}$, S.H Stark ${ }^{40}$, P. Staroba ${ }^{141}$, P. Starovoitov ${ }^{61 a}$, S. Stärz ${ }^{103}$, R. Staszewski ${ }^{84}$, G. Stavropoulos ${ }^{44}$, M. Stegler ${ }^{46}$, P. Steinberg ${ }^{29}$, B. Stelzer ${ }^{152}$, H.J. Stelzer ${ }^{36}$, O. Stelzer-Chilton ${ }^{168 a}$, H. Stenzel ${ }^{56}$, T.J. Stevenson ${ }^{156}$, G.A. Stewart ${ }^{36}$, M.C. Stockton ${ }^{36}$, G. Stoicea ${ }^{27 b}$, M. Stolarski ${ }^{140 a}$, P. Stolte ${ }^{53}$, S. Stonjek ${ }^{115}$, A. Straessner ${ }^{48}$, J. Strandberg ${ }^{154}$, S. Strandberg ${ }^{45 a, 45 b}$, M. Strauss ${ }^{128}$, P. Strizenec ${ }^{28 b}$, R. Ströhmer ${ }^{177}$, D.M. Strom ${ }^{131}$, R. Stroynowski ${ }^{42}$, A. Strubig ${ }^{50}$, S.A. Stucci ${ }^{29}$, B. Stugu ${ }^{17}$, J. Stupak ${ }^{128}$, N.A. Styles ${ }^{46}$, D. Su${ }^{153}$, S. Suchek ${ }^{61 a}$, Y. Sugaya ${ }^{133}$, V.V. Sulin ${ }^{110}$, M.J. Sullivan ${ }^{90}$, D.M.S. Sultan ${ }^{54}$, S. Sultansoy ${ }^{4 c}$, T. Sumida ${ }^{85}$, S. Sun ${ }^{105}$, X. Sun ${ }^{3}$, K. Suruliz ${ }^{156}$, C.J.E. Suster ${ }^{157}$, M.R. Sutton ${ }^{156}$, S. Suzuki ${ }^{81}$, M. Svatos ${ }^{141}$, M. Swiatlowski ${ }^{37}$, S.P. Swift ${ }^{2}$, A. Sydorenko ${ }^{99}$, I. Sykora ${ }^{28 a}$, M. Sykora ${ }^{143}$, T. Sykora ${ }^{143}$, D. Ta ${ }^{99}$, K. Tackmann ${ }^{46, y}$,

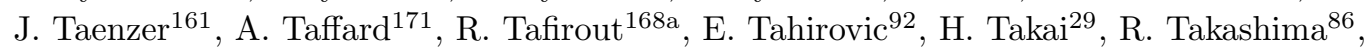
K. Takeda ${ }^{82}$, T. Takeshita ${ }^{150}$, E.P. Takeva ${ }^{50}$, Y. Takubo ${ }^{81}$, M. Talby ${ }^{101}$, A.A. Talyshever ${ }^{122 b, 122 a}$, J. Tanaka ${ }^{163}$, M. Tanaka ${ }^{165}$, R. Tanaka ${ }^{132}$, B.B. Tannenwald ${ }^{126}$, S. Tapia Araya ${ }^{173}$, S. Tapprogge ${ }^{99}$, A. Tarek Abouelfadl Mohamed ${ }^{136}$, S. Tarem ${ }^{160}$, G. Tarna ${ }^{27 b, c}$, G.F. Tartarelli ${ }^{68 a}$,

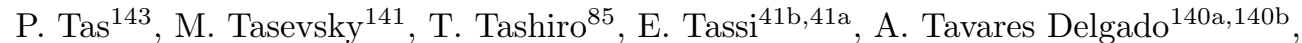
Y. Tayalati ${ }^{35 e}$, A.J. Taylor ${ }^{50}$, G.N. Taylor ${ }^{104}$, P.T.E. Taylor ${ }^{104}$, W. Taylor ${ }^{168 b}$, A.S. Tee ${ }^{89}$, R. Teixeira De Lima ${ }^{153}$, P. Teixeira-Dias ${ }^{93}$, H. Ten Kate ${ }^{36}$, J.J. Teoh ${ }^{120}$, S. Terada ${ }^{81}$,

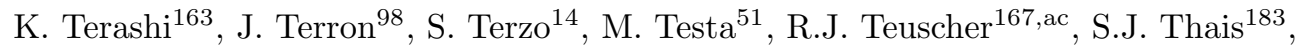

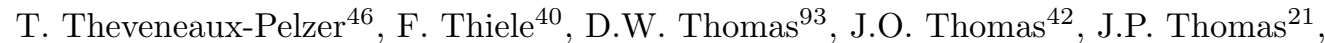
A.S. Thompson ${ }^{57}$, P.D. Thompson ${ }^{21}$, L.A. Thomsen ${ }^{183}$, E. Thomson ${ }^{137}$, Y. Tian ${ }^{39}$, R.E. Ticse Torres $^{53}$, V.O. Tikhomirov ${ }^{110, a o}$, Yu.A. Tikhonov ${ }^{122 b, 122 a}$, S. Timoshenko ${ }^{112}$, P. Tipton ${ }^{183}$, S. Tisserant ${ }^{101}$, K. Todome ${ }^{23 \mathrm{~b}, 23 \mathrm{a}}$, S. Todorova-Nova ${ }^{5}$, S. Todt ${ }^{48}$, J. Tojo $^{87}$, S. Tokár ${ }^{28 a}$, K. Tokushuku ${ }^{81}$, E. Tolley ${ }^{126}$, K.G. Tomiwa ${ }^{33 c}$, M. Tomoto ${ }^{117}$, L. Tompkins ${ }^{153, p}$, K. Toms ${ }^{118}$, B. Tong ${ }^{59}$, P. Tornambe ${ }^{102}$, E. Torrence ${ }^{131}$, H. Torres ${ }^{48}$, E. Torró Pastor ${ }^{148}$,

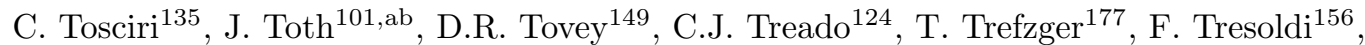
A. Tricoli ${ }^{29}$, I.M. Trigger ${ }^{168 a}$, S. Trincaz-Duvoid ${ }^{136}$, W. Trischuk ${ }^{167}$, B. Trocmé ${ }^{58}$, A. Trofymov ${ }^{132}$, C. Troncon ${ }^{68 a}$, M. Trovatelli ${ }^{176}$, F. Trovato ${ }^{156}$, L. Truong ${ }^{33 b}$, M. Trzebinski ${ }^{84}$, A. Trzupek ${ }^{84}$, F. Tsai ${ }^{46}$, J.C-L. Tseng ${ }^{135}$, P.V. Tsiareshka ${ }^{107, \text { ai }}$, A. Tsirigotis ${ }^{162}$, N. Tsirintanis ${ }^{9}$, V. Tsiskaridze ${ }^{155}$, E.G. Tskhadadze ${ }^{159 a}$, M. Tsopoulou ${ }^{162}$, I.I. Tsukerman ${ }^{111}$, V. Tsulaia ${ }^{18}$,

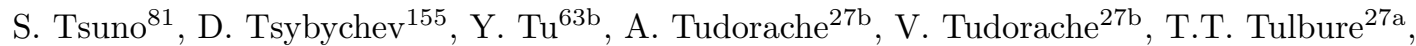
A.N. Tuna ${ }^{59}$, S. Turchikhin ${ }^{79}$, D. Turgeman ${ }^{180}$, I. Turk Cakir ${ }^{4 b, t}$, R.J. Turner ${ }^{21}$, R.T. Turra ${ }^{68 a}$, P.M. Tuts ${ }^{39}$, S Tzamarias ${ }^{162}$, E. Tzovara ${ }^{99}$, G. Ucchielli ${ }^{47}$, I. Ueda ${ }^{81}$, M. Ughetto ${ }^{45 a, 45 b}$,

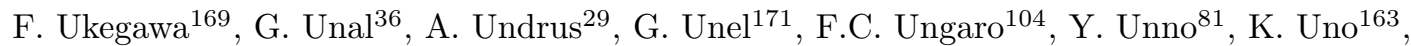

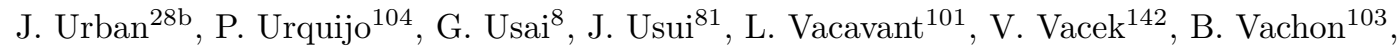
K.O.H. Vadla ${ }^{134}$, A. Vaidya ${ }^{94}$, C. Valderanis ${ }^{114}$, E. Valdes Santurio ${ }^{45 a, 45 b}$, M. Valente ${ }^{54}$, S. Valentinetti ${ }^{23 b, 23 a}$, A. Valero ${ }^{174}$, L. Valéry ${ }^{46}$, R.A. Vallance ${ }^{21}$, A. Vallier $^{36}$, J.A. Valls Ferrer ${ }^{174}$, T.R. Van Daalen ${ }^{14}$, P. Van Gemmeren ${ }^{6}$, I. Van Vulpen ${ }^{120}$, M. Vanadiaa ${ }^{73 a, 73 b}$, W. Vandelli ${ }^{36}$,

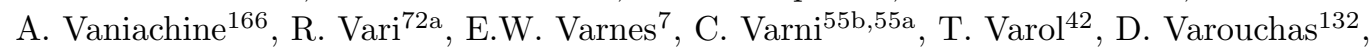
K.E. Varvell ${ }^{157}$, M.E. Vasile ${ }^{27 b}$, G.A. Vasquez ${ }^{176}$, J.G. Vasquez ${ }^{183}$, F. Vazeille ${ }^{38}$, D. Vazquez Furelos ${ }^{14}$, T. Vazquez Schroeder $^{36}$, J. Veatch ${ }^{53}$, V. Vecchio ${ }^{74 a, 74 b}$, L.M. Veloce ${ }^{167}$, F. Veloso ${ }^{140 a, 140 c}$, S. Veneziano ${ }^{72 a}$, A. Ventura ${ }^{67 a, 67 b}, N$. Venturi $^{36}$, A. Verbytskyi ${ }^{115}$, V. Vercesi ${ }^{70 a}$, M. Verducci ${ }^{74 a, 74 b}$, C.M. Vergel Infante ${ }^{78}$, C. Vergis ${ }^{24}$, W. Verkerke ${ }^{120}$, A.T. Vermeulen ${ }^{120}$, J.C. Vermeulen ${ }^{120}$, M.C. Vetterli ${ }^{152, a v}$, N. Viaux Maira ${ }^{147 b}$, M. Vicente Barreto Pinto ${ }^{54}$, I. Vichou ${ }^{173, *}$, T. Vickey ${ }^{149}$, O.E. Vickey Boeriu ${ }^{149}$, G.H.A. Viehhauser ${ }^{135}$, L. Vigani ${ }^{135}$, M. Villa ${ }^{23 b, 23 a}$, M. Villaplana Perez ${ }^{68 a, 68 b}$, E. Vilucchi ${ }^{51}$, M.G. Vincter ${ }^{34}$, V.B. Vinogradov ${ }^{79}$, A. Vishwakarma ${ }^{46}$, C. Vittori ${ }^{23 b, 23 a}$, I. Vivarelli ${ }^{156}$, M. Vogel ${ }^{182}$, P. Vokac ${ }^{142}$, G. Volpi ${ }^{14}$, S.E. von Buddenbrock ${ }^{33 c}$, E. Von Toerne ${ }^{24}$, V. Vorobel ${ }^{143}$, K. Vorobev ${ }^{112}$, M. Vos ${ }^{174}$, J.H. Vossebeld ${ }^{90}$, N. Vranjes ${ }^{16}$, M. Vranjes Milosavljevic ${ }^{16}$, V. Vrba ${ }^{142}$, 
M. Vreeswijk ${ }^{120}$, T. Šfiligoj ${ }^{91}$, R. Vuillermet ${ }^{36}$, I. Vukotic ${ }^{37}$, T. Ženiš ${ }^{28 a}$, L. Živković $^{16}$, P. Wagner ${ }^{24}$, W. Wagner ${ }^{182}$, J. Wagner-Kuhr ${ }^{114}$, H. Wahlberg ${ }^{88}$, S. Wahrmund ${ }^{48}$, K. Wakamiya ${ }^{82}$, V.M. Walbrecht ${ }^{115}$, J. Walder ${ }^{89}$, R. Walker ${ }^{114}$, S.D. Walker ${ }^{93}$, W. Walkowiak ${ }^{151}$, V. Wallangen ${ }^{45 a, 45 b}$, A.M. Wang ${ }^{59}$, C. Wang ${ }^{60 b}$, F. Wang ${ }^{181}$, H. Wang ${ }^{18}$, H. Wang ${ }^{3}$, J. Wang ${ }^{157}$, J. Wang ${ }^{61 b}$, P. Wang ${ }^{42}$, Q. Wang ${ }^{128}$, R.-J. Wang ${ }^{136}$, R. Wang ${ }^{60 a}$, R. Wang ${ }^{6}$, S.M. Wang ${ }^{158}$,

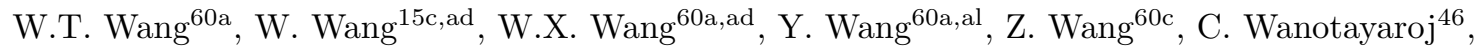
A. Warburton ${ }^{103}$, C.P. Ward ${ }^{32}$, D.R. Wardrope ${ }^{94}$, A. Washbrook ${ }^{50}$, A.T. Watson ${ }^{21}$, M.F. Watson ${ }^{21}$, G. Watts ${ }^{148}$, B.M. Waugh ${ }^{94}$, A.F. Webb ${ }^{11}$, S. Webb ${ }^{99}$, C. Weber ${ }^{183}$, M.S. Weber ${ }^{20}$, S.A. Weber ${ }^{34}$, S.M. Weber ${ }^{61 a}$, A.R. Weidberg ${ }^{135}$, J. Weingarten ${ }^{47}$, M. Weirich ${ }^{99}$, C. Weiser ${ }^{52}$, P.S. Wells ${ }^{36}$, T. Wenaus ${ }^{29}$, T. Wengler ${ }^{36}$, S. Wenig ${ }^{36}$, N. Wermes ${ }^{24}$, M.D. Werner ${ }^{78}$, P. Werner ${ }^{36}$, M. Wessels ${ }^{61 a}$, T.D. Weston ${ }^{20}$, K. Whalen ${ }^{131}$, N.L. Whallon ${ }^{148}$, A.M. Wharton ${ }^{89}$, A.S. White ${ }^{105}$, A. White ${ }^{8}$, M.J. White ${ }^{1}$, R. White ${ }^{147 b}$, D. Whiteson ${ }^{171}$, B.W. Whitmore ${ }^{89}$, F.J. Wickens ${ }^{144}$, W. Wiedenmann ${ }^{181}$, M. Wielers ${ }^{144}$, C. Wiglesworth ${ }^{40}$, L.A.M. Wiik-Fuchs ${ }^{52}$, F. Wilk ${ }^{100}$, H.G. Wilkens ${ }^{36}$, L.J. Wilkins ${ }^{93}$, H.H. Williams ${ }^{137}$, S. Williams ${ }^{32}$, C. Willis ${ }^{106}$, S. Willocq ${ }^{102}$, J.A. Wilson ${ }^{21}$, I. Wingerter-Seez ${ }^{5}$, E. Winkels ${ }^{156}$, F. Winklmeier ${ }^{131}$, O.J. Winston ${ }^{156}$, B.T. Winter ${ }^{52}$, M. Wittgen ${ }^{153}$, M. Wobisch ${ }^{95}$, A. Wolf ${ }^{99}$, T.M.H. Wolf ${ }^{120}$, R. Wolff ${ }^{101}$, R.W. Wölker ${ }^{135}$, J. Wollrath ${ }^{52}$, M.W. Wolter ${ }^{84}$, H. Wolters ${ }^{140 a, 140 c}$, V.W.S. Wong ${ }^{175}$, N.L. Woods ${ }^{146}$, S.D. Worm ${ }^{21}$, B.K. Wosiek ${ }^{84}$, K.W. Woźniak ${ }^{84}$, K. Wraight ${ }^{57}$, S.L. Wu ${ }^{181}$, X. Wu ${ }^{54}$, Y. Wu ${ }^{60 a}$, T.R. Wyatt ${ }^{100}$, B.M. Wynne ${ }^{50}$, S. Xella ${ }^{40}$, Z. Xi ${ }^{105}$, L. Xia $^{178}$, D. Xu ${ }^{15 a}$, H. Xu ${ }^{60 a, c}$, L. Xu ${ }^{29}$, T. Xu ${ }^{145}$, W. Xu ${ }^{105}$, Z. Xu ${ }^{60 b}$, Z. Xu ${ }^{153}$, B. Yabsley ${ }^{157}$, S. Yacoob ${ }^{33 a}$, K. Yajima ${ }^{133}$, D.P. Yallup ${ }^{94}$, D. Yamaguchi ${ }^{165}$, Y. Yamaguchi ${ }^{165}$, A. Yamamoto ${ }^{81}$,

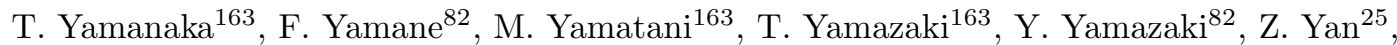

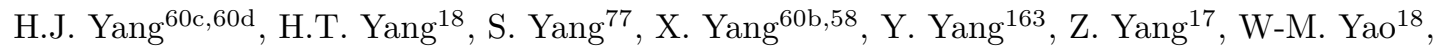
Y.C. Yap ${ }^{46}$, Y. Yasu ${ }^{81}$, E. Yatsenko ${ }^{60 c, 60 d}$, J. Ye ${ }^{42}$, S. Ye ${ }^{29}$, I. Yeletskikh ${ }^{79}$, E. Yigitbasi $^{25}$, E. Yildirim ${ }^{99}$, K. Yorita ${ }^{179}$, K. Yoshihara ${ }^{137}$, C.J.S. Young ${ }^{36}$, C. Young ${ }^{153}$, J. Yu $^{78}$, X. Yue ${ }^{61 a}$, S.P.Y. Yuen ${ }^{24}$, B. Zabinski ${ }^{84}$, G. Zacharis ${ }^{10}$, E. Zaffaroni ${ }^{54}$, J. Zahreddine ${ }^{136}$, R. Zaidan ${ }^{14}$, A.M. Zaitsev ${ }^{123, a n}$, T. Zakareishvili1 ${ }^{159 b}$, N. Zakharchuk ${ }^{34}$, S. Zambito ${ }^{59}$, D. Zanzi ${ }^{36}$, D.R. Zaripovas ${ }^{57}$, S.V. Zeißner ${ }^{47}$, C. Zeitnitz ${ }^{182}$, G. Zemaityte ${ }^{135}$, J.C. Zeng ${ }^{173}$, O. Zenin ${ }^{123}$, D. Zerwas ${ }^{132}$, M. Zgubič ${ }^{135}$, D.F. Zhang ${ }^{15 b}$, F. Zhang ${ }^{181}$, G. Zhang ${ }^{60 a}$, G. Zhang ${ }^{15 b}$, H. Zhang ${ }^{15 c}$, J. Zhang ${ }^{6}$, L. Zhang ${ }^{15 c}$, L. Zhang ${ }^{60 a}$, M. Zhang ${ }^{173}$, R. Zhang ${ }^{60 a}$, R. Zhang ${ }^{24}$, X. Zhang ${ }^{60 b}$, Y. Zhang ${ }^{15 a, 15 d}$, Z. Zhang ${ }^{63 a}$, Z. Zhang ${ }^{132}$, P. Zhao ${ }^{49}$, Y. Zhao ${ }^{60 b}$, Z. Zhao $^{60 a}$, A. Zhemchugov ${ }^{79}$, Z. Zheng ${ }^{105}$, D. Zhong ${ }^{173}$, B. Zhou ${ }^{105}$, C. Zhou ${ }^{181}$, M.S. Zhou ${ }^{15 a, 15 d}$, M. Zhou ${ }^{155}$, N. Zhou ${ }^{60 c}$, Y. Zhou ${ }^{7}$, C.G. Zhu ${ }^{60 b}$, H.L. Zhu ${ }^{60 a}$, H. Zhu ${ }^{15 a}$, J. Zhu ${ }^{105}$, Y. Zhu ${ }^{60 a}$, X. Zhuang ${ }^{15 a}$, K. Zhukov ${ }^{110}$, V. Zhulanov ${ }^{122 b, 122 a}$, D. Zieminska ${ }^{65}$, N.I. Zimine ${ }^{79}$, S. Zimmermann ${ }^{52}$, Z. Zinonos ${ }^{115}$, M. Ziolkowski ${ }^{151}$, G. Zobernig ${ }^{181}$, A. Zoccoli ${ }^{23 b, 23 a}$, K. Zoch ${ }^{53}$, T.G. Zorbas ${ }^{149}$, R. Zou ${ }^{37}$, L. Zwalinski ${ }^{36}$

1 Department of Physics, University of Adelaide, Adelaide; Australia

2 Physics Department, SUNY Albany, Albany NY; United States of America

3 Department of Physics, University of Alberta, Edmonton AB; Canada

$4{ }^{(a)}$ Department of Physics, Ankara University, Ankara ${ }^{(b)}$, Istanbul Aydin University, Istanbul ${ }^{(c)}$, Division of Physics, TOBB University of Economics and Technology, Ankara; Turkey

5 LAPP, Université Grenoble Alpes, Université Savoie Mont Blanc, CNRS/IN2P3, Annecy; France

6 High Energy Physics Division, Argonne National Laboratory, Argonne IL; United States of America

7 Department of Physics, University of Arizona, Tucson AZ; United States of America

8 Department of Physics, University of Texas at Arlington, Arlington TX; United States of America

9 Physics Department, National and Kapodistrian University of Athens, Athens; Greece

10 Physics Department, National Technical University of Athens, Zografou; Greece

11 Department of Physics, University of Texas at Austin, Austin TX; United States of America 
$12{ }^{(a)}$ Bahcesehir University, Faculty of Engineering and Natural Sciences, Istanbul ${ }^{(b)}$, Istanbul Bilgi University, Faculty of Engineering and Natural Sciences, Istanbul ${ }^{(c)}$, Department of Physics, Bogazici University, Istanbul ${ }^{(d)}$, Department of Physics Engineering, Gaziantep University, Gaziantep; Turkey

13 Institute of Physics, Azerbaijan Academy of Sciences, Baku; Azerbaijan

14 Institut de Física d'Altes Energies (IFAE), Barcelona Institute of Science and Technology, Barcelona; Spain

$15{ }^{(a)}$ Institute of High Energy Physics, Chinese Academy of Sciences, Beijing ${ }^{(b)}$, Physics Department, Tsinghua University, Beijing ${ }^{(c)}$, Department of Physics, Nanjing University, Nanjing ${ }^{(d)}$, University of Chinese Academy of Science (UCAS), Beijing; China

16 Institute of Physics, University of Belgrade, Belgrade; Serbia

17 Department for Physics and Technology, University of Bergen, Bergen; Norway

18 Physics Division, Lawrence Berkeley National Laboratory and University of California, Berkeley CA; United States of America

19 Institut für Physik, Humboldt Universität zu Berlin, Berlin; Germany

20 Albert Einstein Center for Fundamental Physics and Laboratory for High Energy Physics, University of Bern, Bern; Switzerland

21 School of Physics and Astronomy, University of Birmingham, Birmingham; United Kingdom

22 Facultad de Ciencias y Centro de Investigaciónes, Universidad Antonio Nariño, Bogota; Colombia

$23{ }^{(a)}$ INFN Bologna and Universita' di Bologna, Dipartimento di Fisica ${ }^{(b)}$, INFN Sezione di Bologna; Italy

24 Physikalisches Institut, Universität Bonn, Bonn; Germany

25 Department of Physics, Boston University, Boston MA; United States of America

26 Department of Physics, Brandeis University, Waltham MA; United States of America

$27{ }^{(a)}$ Transilvania University of Brasov, Brasov ${ }^{(b)}$, Horia Hulubei National Institute of Physics and Nuclear Engineering, Bucharest ${ }^{(c)}$, Department of Physics, Alexandru Ioan Cuza University of Iasi, Iasi ${ }^{(d)}$, National Institute for Research and Development of Isotopic and Molecular Technologies, Physics Department, Cluj-Napoca ${ }^{(e)}$, University Politehnica Bucharest, Bucharest ${ }^{(f)}$, West University in Timisoara, Timisoara; Romania

$28{ }^{(a)}$ Faculty of Mathematics, Physics and Informatics, Comenius University, Bratislava ${ }^{(b)}$, Department of Subnuclear Physics, Institute of Experimental Physics of the Slovak Academy of Sciences, Kosice; Slovak Republic

29 Physics Department, Brookhaven National Laboratory, Upton NY; United States of America

30 Departamento de Física, Universidad de Buenos Aires, Buenos Aires; Argentina

31 California State University, CA; United States of America

32 Cavendish Laboratory, University of Cambridge, Cambridge; United Kingdom

$33{ }^{(a)}$ Department of Physics, University of Cape Town, Cape Town ${ }^{(b)}$, Department of Mechanical Engineering Science, University of Johannesburg, Johannesburg ${ }^{(c)}$, School of Physics, University of the Witwatersrand, Johannesburg; South Africa

34 Department of Physics, Carleton University, Ottawa ON; Canada

35 (a) Faculté des Sciences Ain Chock, Réseau Universitaire de Physique des Hautes Energies Université Hassan II, Casablanca ${ }^{(b)}$, Faculté des Sciences, Université Ibn-Tofail, Kénitra ${ }^{(c)}$, Faculté des Sciences Semlalia, Université Cadi Ayyad, LPHEA-Marrakech ${ }^{(d)}$, Faculté des Sciences, Université Mohamed Premier and LPTPM, Oujda ${ }^{(e)}$, Faculté des sciences, Université Mohammed $V$, Rabat; Morocco

36 CERN, Geneva; Switzerland

37 Enrico Fermi Institute, University of Chicago, Chicago IL; United States of America

38 LPC, Université Clermont Auvergne, CNRS/IN2P3, Clermont-Ferrand; France

39 Nevis Laboratory, Columbia University, Irvington NY; United States of America

40 Niels Bohr Institute, University of Copenhagen, Copenhagen; Denmark

$41{ }^{(a)}$ Dipartimento di Fisica, Università della Calabria, Rende ${ }^{(b)}$, INFN Gruppo Collegato di Cosenza, Laboratori Nazionali di Frascati; Italy 
Physics Department, Southern Methodist University, Dallas TX; United States of America

National Centre for Scientific Research "Demokritos", Agia Paraskevi; Greece

$45{ }^{(a)}$ Department of Physics, Stockholm University ${ }^{(b)}$, Oskar Klein Centre, Stockholm; Sweden

46 Deutsches Elektronen-Synchrotron DESY, Hamburg and Zeuthen; Germany Institut für Kern und Teilchenphysik, Technische Universität Dresden, Dresden; Germany

49 Department of Physics, Duke University, Durham NC; United States of America

50 SUPA - School of Physics and Astronomy, University of Edinburgh, Edinburgh; United Kingdom

51 INFN e Laboratori Nazionali di Frascati, Frascati; Italy

52 Physikalisches Institut, Albert-Ludwigs-Universität Freiburg, Freiburg; Germany

53 II. Physikalisches Institut, Georg-August-Universität Göttingen, Göttingen; Germany

54 Département de Physique Nucléaire et Corpusculaire, Université de Genève, Genève; Switzerland

55 (a) Dipartimento di Fisica, Università di Genova, Genova ${ }^{(b)}$, INFN Sezione di Genova; Italy

56 II. Physikalisches Institut, Justus-Liebig-Universität Giessen, Giessen; Germany

57 SUPA — School of Physics and Astronomy, University of Glasgow, Glasgow; United Kingdom

58 LPSC, Université Grenoble Alpes, CNRS/IN2P3, Grenoble INP, Grenoble; France

59 Laboratory for Particle Physics and Cosmology, Harvard University, Cambridge MA; United States of America

60 (a) Department of Modern Physics and State Key Laboratory of Particle Detection and Electronics, University of Science and Technology of China, Hefei ${ }^{(b)}$, Institute of Frontier and Interdisciplinary Science and Key Laboratory of Particle Physics and Particle Irradiation (MOE), Shandong University, Qingdao ${ }^{(c)}$, School of Physics and Astronomy, Shanghai Jiao Tong University, KLPPAC-MoE, SKLPPC, Shanghai ${ }^{(d)}$, Tsung-Dao Lee Institute, Shanghai; China

$61{ }^{(a)}$ Kirchhoff-Institut für Physik, Ruprecht-Karls-Universität Heidelberg, Heidelberg ${ }^{(b)}$, Physikalisches Institut, Ruprecht-Karls-Universität Heidelberg, Heidelberg; Germany

62 Faculty of Applied Information Science, Hiroshima Institute of Technology, Hiroshima; Japan

$63{ }^{(a)}$ Department of Physics, Chinese University of Hong Kong, Shatin, N.T., Hong Kong ${ }^{(b)}$, Department of Physics, University of Hong Kong, Hong Kong ${ }^{(c)}$, Department of Physics and Institute for Advanced Study, Hong Kong University of Science and Technology, Clear Water Bay, Kowloon, Hong Kong; China

64 Department of Physics, National Tsing Hua University, Hsinchu; Taiwan

65 Department of Physics, Indiana University, Bloomington IN; United States of America

$66{ }^{(a)}$ INFN Gruppo Collegato di Udine, Sezione di Trieste, Udine ${ }^{(b)}$, ICTP, Trieste ${ }^{(c)}$, Dipartimento Politecnico di Ingegneria e Architettura, Università di Udine, Udine; Italy

$67{ }^{(a)}$ INFN Sezione di Lecce ${ }^{(b)}$, Dipartimento di Matematica e Fisica, Università del Salento, Lecce; Italy

$68{ }^{(a)}$ INFN Sezione di Milano ${ }^{(b)}$, Dipartimento di Fisica, Università di Milano, Milano; Italy

$69{ }^{(a)}$ INFN Sezione di Napoli ${ }^{(b)}$, Dipartimento di Fisica, Università di Napoli, Napoli; Italy

70 (a) INFN Sezione di Pavia ${ }^{(b)}$, Dipartimento di Fisica, Università di Pavia, Pavia; Italy

$71{ }^{(a)}$ INFN Sezione di Pisa ${ }^{(b)}$, Dipartimento di Fisica E. Fermi, Università di Pisa, Pisa; Italy

$72{ }^{(a)}$ INFN Sezione di Roma ${ }^{(b)}$, Dipartimento di Fisica, Sapienza Università di Roma, Roma; Italy

$73{ }^{(a)}$ INFN Sezione di Roma Tor Vergata ${ }^{(b)}$, Dipartimento di Fisica, Università di Roma Tor Vergata, Roma; Italy

74 (a) INFN Sezione di Roma Tre ${ }^{(b)}$, Dipartimento di Matematica e Fisica, Università Roma Tre, Roma; Italy

$75{ }^{(a)}$ INFN-TIFPA ${ }^{(b)}$, Università degli Studi di Trento, Trento; Italy

76 Institut für Astro und Teilchenphysik, Leopold-Franzens-Universität, Innsbruck; Austria

77 University of Iowa, Iowa City IA; United States of America

78 Department of Physics and Astronomy, Iowa State University, Ames IA; United States of America

79 Joint Institute for Nuclear Research, Dubna; Russia 
80 (a) Departamento de Engenharia Elétrica, Universidade Federal de Juiz de Fora (UFJF), Juiz de Fora $^{(b)}$, Universidade Federal do Rio De Janeiro COPPE/EE/IF, Rio de Janeiro ${ }^{(c)}$, Universidade Federal de São João del Rei (UFSJ), São João del Rei ${ }^{(d)}$, Instituto de Física, Universidade de São Paulo, São Paulo; Brazil

81 KEK, High Energy Accelerator Research Organization, Tsukuba; Japan

82 Graduate School of Science, Kobe University, Kobe; Japan

$83{ }^{(a)}$ AGH University of Science and Technology, Faculty of Physics and Applied Computer Science, Krakow $^{(b)}$, Marian Smoluchowski Institute of Physics, Jagiellonian University, Krakow; Poland

84 Institute of Nuclear Physics Polish Academy of Sciences, Krakow; Poland

85 Faculty of Science, Kyoto University, Kyoto; Japan

86 Kyoto University of Education, Kyoto; Japan

87 Research Center for Advanced Particle Physics and Department of Physics, Kyushu University, Fukuoka; Japan

88 Instituto de Física La Plata, Universidad Nacional de La Plata and CONICET, La Plata; Argentina

89 Physics Department, Lancaster University, Lancaster; United Kingdom

90 Oliver Lodge Laboratory, University of Liverpool, Liverpool; United Kingdom

91 Department of Experimental Particle Physics, Jožef Stefan Institute and Department of Physics, University of Ljubljana, Ljubljana; Slovenia

92 School of Physics and Astronomy, Queen Mary University of London, London; United Kingdom

93 Department of Physics, Royal Holloway University of London, Egham; United Kingdom

94 Department of Physics and Astronomy, University College London, London; United Kingdom

95 Louisiana Tech University, Ruston LA; United States of America

96 Fysiska institutionen, Lunds universitet, Lund; Sweden

97 Centre de Calcul de l'Institut National de Physique Nucléaire et de Physique des Particules (IN2P3), Villeurbanne; France

98 Departamento de Física Teorica C-15 and CIAFF, Universidad Autónoma de Madrid, Madrid; Spain

99 Institut für Physik, Universität Mainz, Mainz; Germany

100 School of Physics and Astronomy, University of Manchester, Manchester; United Kingdom

101 CPPM, Aix-Marseille Université, CNRS/IN2P3, Marseille; France

102 Department of Physics, University of Massachusetts, Amherst MA; United States of America

103 Department of Physics, McGill University, Montreal QC; Canada

104 School of Physics, University of Melbourne, Victoria; Australia

105 Department of Physics, University of Michigan, Ann Arbor MI; United States of America

106 Department of Physics and Astronomy, Michigan State University, East Lansing MI; United States of America

107 B.I. Stepanov Institute of Physics, National Academy of Sciences of Belarus, Minsk; Belarus

108 Research Institute for Nuclear Problems of Byelorussian State University, Minsk; Belarus

109 Group of Particle Physics, University of Montreal, Montreal QC; Canada

110 P.N. Lebedev Physical Institute of the Russian Academy of Sciences, Moscow; Russia

111 Institute for Theoretical and Experimental Physics of the National Research Centre Kurchatov Institute, Moscow; Russia

112 National Research Nuclear University MEPhI, Moscow; Russia

113 D.V. Skobeltsyn Institute of Nuclear Physics, M.V. Lomonosov Moscow State University, Moscow; Russia

114 Fakultät für Physik, Ludwig-Maximilians-Universität München, München; Germany

115 Max-Planck-Institut für Physik (Werner-Heisenberg-Institut), München; Germany

116 Nagasaki Institute of Applied Science, Nagasaki; Japan

117 Graduate School of Science and Kobayashi-Maskawa Institute, Nagoya University, Nagoya; Japan

118 Department of Physics and Astronomy, University of New Mexico, Albuquerque NM; United States of America

119 Institute for Mathematics, Astrophysics and Particle Physics, Radboud University Nijmegen/Nikhef, Nijmegen; Netherlands 
Nikhef National Institute for Subatomic Physics and University of Amsterdam, Amsterdam; Netherlands

121 Department of Physics, Northern Illinois University, DeKalb IL; United States of America

$122{ }^{(a)}$ Budker Institute of Nuclear Physics and NSU, SB RAS, Novosibirsk ${ }^{(b)}$, Novosibirsk State University Novosibirsk; Russia

123 Institute for High Energy Physics of the National Research Centre Kurchatov Institute, Protvino; Russia

124 Department of Physics, New York University, New York NY; United States of America

125 Ochanomizu University, Otsuka, Bunkyo-ku, Tokyo; Japan

126 Ohio State University, Columbus OH; United States of America

127 Faculty of Science, Okayama University, Okayama; Japan

128 Homer L. Dodge Department of Physics and Astronomy, University of Oklahoma, Norman OK; United States of America

129 Department of Physics, Oklahoma State University, Stillwater OK; United States of America

130 Palacký University, RCPTM, Joint Laboratory of Optics, Olomouc; Czech Republic

131 Center for High Energy Physics, University of Oregon, Eugene OR; United States of America

${ }^{132}$ LAL, Université Paris-Sud, CNRS/IN2P3, Université Paris-Saclay, Orsay; France

133 Graduate School of Science, Osaka University, Osaka; Japan

134 Department of Physics, University of Oslo, Oslo; Norway

135 Department of Physics, Oxford University, Oxford; United Kingdom

${ }^{136}$ LPNHE, Sorbonne Université, Paris Diderot Sorbonne Paris Cité, CNRS/IN2P3, Paris; France

137 Department of Physics, University of Pennsylvania, Philadelphia PA; United States of America

138 Konstantinov Nuclear Physics Institute of National Research Centre "Kurchatov Institute", PNPI, St. Petersburg; Russia

139 Department of Physics and Astronomy, University of Pittsburgh, Pittsburgh PA; United States of America

$140{ }^{(a)}$ Laboratório de Instrumentação e Física Experimental de Partículas - LIP ${ }^{(b)}$, Departamento de Física, Faculdade de Ciências, Universidade de Lisboa, Lisboa ${ }^{(c)}$, Departamento de Física, Universidade de Coimbra, Coimbra ${ }^{(d)}$, Centro de Física Nuclear da Universidade de Lisboa, Lisboa $^{(e)}$, Departamento de Física, Universidade do Minho, Braga ${ }^{(f)}$, Universidad de Granada, Granada $(\text { Spain })^{(g)}$, Dep Física and CEFITEC of Faculdade de Ciências e Tecnologia, Universidade Nova de Lisboa, Caparica; Portugal

141 Institute of Physics of the Czech Academy of Sciences, Prague; Czech Republic

142 Czech Technical University in Prague, Prague; Czech Republic

143 Charles University, Faculty of Mathematics and Physics, Prague; Czech Republic

144 Particle Physics Department, Rutherford Appleton Laboratory, Didcot; United Kingdom

145 IRFU, CEA, Université Paris-Saclay, Gif-sur-Yvette; France

146 Santa Cruz Institute for Particle Physics, University of California Santa Cruz, Santa Cruz CA; United States of America

$147{ }^{(a)}$ Departamento de Física, Pontificia Universidad Católica de Chile, Santiago ${ }^{(b)}$, Departamento de Física, Universidad Técnica Federico Santa María, Valparaíso; Chile

148 Department of Physics, University of Washington, Seattle WA; United States of America

149 Department of Physics and Astronomy, University of Sheffield, Sheffield; United Kingdom

150 Department of Physics, Shinshu University, Nagano; Japan

151 Department Physik, Universität Siegen, Siegen; Germany

152 Department of Physics, Simon Fraser University, Burnaby BC; Canada

153 SLAC National Accelerator Laboratory, Stanford CA; United States of America

154 Physics Department, Royal Institute of Technology, Stockholm; Sweden

155 Departments of Physics and Astronomy, Stony Brook University, Stony Brook NY; United States of America

156 Department of Physics and Astronomy, University of Sussex, Brighton; United Kingdom

157 School of Physics, University of Sydney, Sydney; Australia 
158 Institute of Physics, Academia Sinica, Taipei; Taiwan

$159{ }^{(a)}$ E. Andronikashvili Institute of Physics, Iv. Javakhishvili Tbilisi State University, Tbilisi ${ }^{(b)}$, High Energy Physics Institute, Tbilisi State University, Tbilisi; Georgia

160 Department of Physics, Technion, Israel Institute of Technology, Haifa; Israel

161 Raymond and Beverly Sackler School of Physics and Astronomy, Tel Aviv University, Tel Aviv; Israel

162 Department of Physics, Aristotle University of Thessaloniki, Thessaloniki; Greece

163 International Center for Elementary Particle Physics and Department of Physics, University of Tokyo, Tokyo; Japan

164 Graduate School of Science and Technology, Tokyo Metropolitan University, Tokyo; Japan

165 Department of Physics, Tokyo Institute of Technology, Tokyo; Japan

166 Tomsk State University, Tomsk; Russia

167 Department of Physics, University of Toronto, Toronto ON; Canada

$168{ }^{(a)}$ TRIUMF, Vancouver BC ${ }^{(b)}$, Department of Physics and Astronomy, York University, Toronto ON; Canada

169 Division of Physics and Tomonaga Center for the History of the Universe, Faculty of Pure and Applied Sciences, University of Tsukuba, Tsukuba; Japan

170 Department of Physics and Astronomy, Tufts University, Medford MA; United States of America

171 Department of Physics and Astronomy, University of California Irvine, Irvine CA; United States of America

172 Department of Physics and Astronomy, University of Uppsala, Uppsala; Sweden

173 Department of Physics, University of Illinois, Urbana IL; United States of America

174 Instituto de Física Corpuscular (IFIC), Centro Mixto Universidad de Valencia - CSIC, Valencia; Spain

175 Department of Physics, University of British Columbia, Vancouver BC; Canada

176 Department of Physics and Astronomy, University of Victoria, Victoria BC; Canada

177 Fakultät für Physik und Astronomie, Julius-Maximilians-Universität Würzburg, Würzburg; Germany

178 Department of Physics, University of Warwick, Coventry; United Kingdom

179 Waseda University, Tokyo; Japan

180 Department of Particle Physics, Weizmann Institute of Science, Rehovot; Israel

181 Department of Physics, University of Wisconsin, Madison WI; United States of America

182 Fakultät für Mathematik und Naturwissenschaften, Fachgruppe Physik, Bergische Universität Wuppertal, Wuppertal; Germany

183 Department of Physics, Yale University, New Haven CT; United States of America

184 Yerevan Physics Institute, Yerevan; Armenia

a Also at Centre for High Performance Computing, CSIR Campus, Rosebank, Cape Town; South Africa

${ }^{b}$ Also at CERN, Geneva; Switzerland

c Also at CPPM, Aix-Marseille Université, CNRS/IN2P3, Marseille; France

d Also at Département de Physique Nucléaire et Corpusculaire, Université de Genève, Genève; Switzerland

e Also at Departament de Fisica de la Universitat Autonoma de Barcelona, Barcelona; Spain

${ }^{f}$ Also at Departamento de Física, Instituto Superior Técnico, Universidade de Lisboa, Lisboa; Portugal

$g$ Also at Department of Applied Physics and Astronomy, University of Sharjah, Sharjah; United Arab Emirates

${ }^{h}$ Also at Department of Financial and Management Engineering, University of the Aegean, Chios; Greece

i Also at Department of Physics and Astronomy, University of Louisville, Louisville, KY; United States of America 
$j$ Also at Department of Physics and Astronomy, University of Sheffield, Sheffield; United Kingdom

${ }^{k}$ Also at Department of Physics, California State University, East Bay; United States of America

$l$ Also at Department of Physics, California State University, Fresno; United States of America

$m$ Also at Department of Physics, California State University, Sacramento; United States of America

$n$ Also at Department of Physics, King's College London, London; United Kingdom

- Also at Department of Physics, St. Petersburg State Polytechnical University, St. Petersburg; Russia

$p$ Also at Department of Physics, Stanford University, Stanford CA; United States of America

$q$ Also at Department of Physics, University of Fribourg, Fribourg; Switzerland

$r$ Also at Department of Physics, University of Michigan, Ann Arbor MI; United States of America

$s$ Also at Faculty of Physics, M.V. Lomonosov Moscow State University, Moscow; Russia

${ }^{t}$ Also at Giresun University, Faculty of Engineering, Giresun; Turkey

u Also at Graduate School of Science, Osaka University, Osaka; Japan

$\checkmark \quad$ Also at Hellenic Open University, Patras; Greece

w Also at Horia Hulubei National Institute of Physics and Nuclear Engineering, Bucharest; Romania

$x$ Also at Institucio Catalana de Recerca i Estudis Avancats, ICREA, Barcelona; Spain

$y$ Also at Institut für Experimentalphysik, Universität Hamburg, Hamburg; Germany

$z$ Also at Institute for Mathematics, Astrophysics and Particle Physics, Radboud University Nijmegen/Nikhef, Nijmegen; Netherlands

a a Also at Institute for Nuclear Research and Nuclear Energy (INRNE) of the Bulgarian Academy of Sciences, Sofia; Bulgaria

ab Also at Institute for Particle and Nuclear Physics, Wigner Research Centre for Physics, Budapest; Hungary

ac Also at Institute of Particle Physics (IPP); Canada

ad Also at Institute of Physics, Academia Sinica, Taipei; Taiwan

ae Also at Institute of Physics, Azerbaijan Academy of Sciences, Baku; Azerbaijan

af Also at Institute of Theoretical Physics, Ilia State University, Tbilisi; Georgia

ag Also at Instituto de Fisica Teorica, IFT-UAM/CSIC, Madrid; Spain

ah Also at Istanbul University, Dept. of Physics, Istanbul; Turkey

ai Also at Joint Institute for Nuclear Research, Dubna; Russia

aj Also at LAL, Université Paris-Sud, CNRS/IN2P3, Université Paris-Saclay, Orsay; France

ak Also at Louisiana Tech University, Ruston LA; United States of America

al Also at LPNHE, Sorbonne Université, Paris Diderot Sorbonne Paris Cité, CNRS/IN2P3, Paris; France

am Also at Manhattan College, New York NY; United States of America

an Also at Moscow Institute of Physics and Technology State University, Dolgoprudny; Russia

ao Also at National Research Nuclear University MEPhI, Moscow; Russia

ap Also at Physics Department, An-Najah National University, Nablus; Palestine

${ }^{a q}$ Also at Physikalisches Institut, Albert-Ludwigs-Universität Freiburg, Freiburg; Germany

ar Also at School of Physics, Sun Yat-sen University, Guangzhou; China

as Also at The City College of New York, New York NY; United States of America

at Also at The Collaborative Innovation Center of Quantum Matter (CICQM), Beijing; China

au Also at Tomsk State University, Tomsk, and Moscow Institute of Physics and Technology State University, Dolgoprudny; Russia

av Also at TRIUMF, Vancouver BC; Canada

aw Also at Universita di Napoli Parthenope, Napoli; Italy

* Deceased 